UNIVERSIDADE DE SÃO PAULO

FACULDADE DE FILOSOFIA, LETRAS E CIÊNCIAS HUMANAS DEPARTAMENTO DE LETRAS MODERNAS

PROGRAMA DE PÓS-GRADUAÇÃO

DA ÁREA DE ESTUDOS LINGUÍSTICOS, LITERÁRIOS E TRADUTOLÓGICOS EM FRANCÊS

SÉBASTIEN ROY

La Voie cruelle d'Ella Maillart : un récit de la fin des voyages

São Paulo 


\author{
UNIVERSIDADE DE SÃO PAULO \\ FACULDADE DE FILOSOFIA, LETRAS E CIÊNCIAS HUMANAS \\ DEPARTAMENTO DE LETRAS MODERNAS \\ PROGRAMA DE PÓS-GRADUAÇÃO \\ DA ÁREA DE ESTUDOS LINGUÍSTICOS, LITERÁRIOS E \\ TRADUTOLÓGICOS EM FRANCÊS
}

\title{
La Voie cruelle d'Ella Maillart : \\ un récit de la fin des voyages
}

\begin{abstract}
Sébastien Roy
Dissertação apresentada ao Programa de PósGraduação da Área de Estudos Linguísticos, Literários e Tradutológicos em Francês do Departamento de Letras Modernas da Faculdade de Filosofia, Letras e Ciências Humanas da Universidade de São Paulo, para a obtenção do título de Mestre em Letras.
\end{abstract}

Orientador:

Profa. Dra. Leyla Perrone-Moisés

São Paulo 
RESUMO: Pretendeu-se que o século XX tinha presenciado o fim das viagens, condenando as narrativas que as contavam a não ser mais do que fábulas. A obra de Ella Maillart e, sobretudo, A Via cruel, relato escrito durante a Segunda Guerra Mundial, testemunham desse momento crítico. Ella Maillart inscreve-se na tradição das relações de viagens, tanto pela escolha de um itinerário seguindo a Rota da Seda, como por uma percepção do espaço asiático no qual situa, como os viajantes da Idade-Média, o Éden ou, pelo menos, uma tribo edênica, graças à qual o Ocidente poderia reencontrar suas origens perdidas. No entanto, a viagem de carro até o Afeganistão leva Ella Maillart a medir quanto, ao revés de uma filiação antiga entre Ásia e Europa ainda visível em certos sinais, o Ocidente aos poucos vai se expandindo pelo Leste, trocando a variedade das culturas por uma civilização industrial baseada na satisfação de necessidades artificiais. Além disso, o encontro tão almejado com o Outro, que leva a nuançar o exotismo de Ella Maillart por um relativismo moderado no qual a evolução das culturas é possível se vier de uma força interna, termina em um fracasso duplo: ou o Outro, como o iraniano já europeizado, ou mesmo o afegão mais preservado, se revela demasiado parecido com o viajante; ou, como os nômades, mostra-lhe sua irredutível alteridade. Assim, frente à impossibilidade de alcançar um Éden geográfico, Ella Maillart desprende-se do mundo objetivo e vira-se para o mundo subjetivo, do qual os diálogos com Annemarie Schwarzenbach (Christina), atormentada pela depressão e pela dependência à morfina, a levam a reconhecer a superioridade, encontrando nele a única viagem possível e, na literatura e na poesia, a única escrita capaz de contá-la. Portanto, ainda que herdeira de uma tradição das relações de viagens remetendo à Idade-Média, A Via cruel revela também seus limites no século XX, abrindo uma via para uma literatura de viagem que, ao contrário dos textos até então escritos tanto por viajantes como por escritores, assuma plenamente sua dimensão literária.

Palavras-chave: Viagem, Ella Maillart, Espaço, Outro, Escrita literária. 
ABSTRACT: The twentieth century was considered the end of travels, thus condemning narratives to be nothing more than fables. Ella Maillart's work and, more particularly, The Cruel Way, written during the Second World War, is a testimony of this crucial moment. Ella Maillart is part of the tradition of travel writers, not only because of the Silk Way itinerary, but also for her perception of the Asian geography which echoes travelers of the Middle Age. Indeed, Asia is where she locates the Garden of Eden or, at least, an idyllic tribe, which would give the opportunity to the West to revive its lost origins. However, contrary to a former filiation between Asia and Europe still visible in certain ways, the journey by car to Afghanistan allows Ella Maillart to assess how much the West slowly expands to the East, changing the variety of cultures for an industrial civilization based on the satisfaction of artificial needs. Moreover, her longing for the Other ends in a double failure : either the Other appears too similar to the traveler, just like the Iranian who is already quite European, or even the Afghan whom she thought protected; or like the nomads who show how her otherness doesn't fit in with their way of living. This encounter will get Ella Maillart to change her exotic vision into a moderated relativism in which the evolution of cultures is possible when driven by an internal force. Therefore, because she is unable to reach a Garden of Eden, Ella Maillart backs down from the objective world to explore the subjective world. Annemarie Schwarzenbach (Christine) who is tormented by depression and morphine dependency, makes her understand that the subjective world is the only possible journey, and that Literature and Poetry are the only means to relate it. To conclude, The Cruel Way is the link between the Middle Age and twentieth century travel writing, initiating a new form which completely assumes its literary dimension, contrary to the texts written until then by travelers and writers.

KEY WORDS: Travel, Ella Maillart, Space, Other, Literary writing. 
RÉSUMÉ : On a pu prétendre que le XXe siècle avait connu la fin des voyages, condamnant les récits qui en rendaient compte à n'être plus que des fables. Or, l'œuvre d'Ella Maillart et, en particulier, La Voie cruelle, écrit pendant la Seconde Guerre Mondiale, sont des témoins de ce moment critique. Ella Maillart s'inscrit dans la tradition des récits de voyage, tant par le choix d'un itinéraire qui suit la Route de la Soie, que par une perception de l'espace asiatique au cœur duquel, comme les voyageurs du Moyen-Âge, elle situe l'Éden ou, du moins, une tribu édénique, qui permettrait à l'Occident de renouer avec ses origines perdues. Cependant, le voyage en voiture jusqu'en Afghanistan permet à Ella Maillart de mesurer combien, à rebours d'une filiation antique entre l'Asie et l'Europe, encore visible à certains signes, l'Occident gagne peu à peu du terrain vers l'Est, remplaçant la variété des cultures par une civilisation industrielle fondée sur la satisfaction de besoins artificiels. De plus, la rencontre tant désirée avec l'Autre, tout en permettant de nuancer l'exotisme d'Ella Maillart par un relativisme modéré, où l'évolution des cultures est possible si elle vient d'une poussée interne, débouche sur un double échec : ou bien l'Autre, comme l'Iranien déjà européanisé, ou même l'Afghan plus préservé, se révèle en fait trop semblable au voyageur ; ou bien, comme les nomades, il lui montre au contraire son irréductible altérité. Aussi, devant l'impossibilité de rejoindre un Éden géographique, Ella Maillart se détourne-t-elle du monde objectif pour explorer le monde subjectif, dont ses dialogues avec Annemarie Schwarzenbach (Christina), tourmentée par sa dépression et sa dépendance à la morphine, l'amènent à reconnaître la supériorité, trouvant en lui le seul voyage possible et, dans la littérature et la poésie, la seule écriture capable d'en rendre compte. Ainsi, tout en étant héritier d'une tradition des récits de voyages remontant au Moyen-Âge, La Voie cruelle en révèle aussi les limites au XXe siècle, tout en ouvrant la voie pour une littérature de voyage qui, contrairement aux textes écrits jusqu'alors tant par des voyageurs que par des écrivains, assume vraiment sa part littéraire.

MOTS-CLÉS : Voyage, Ella Maillart, Espace, Autre, Écriture littéraire. 


\section{Sommaire}

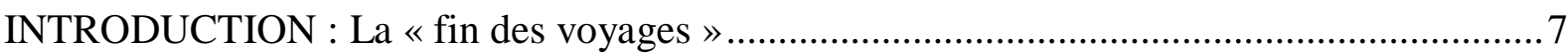

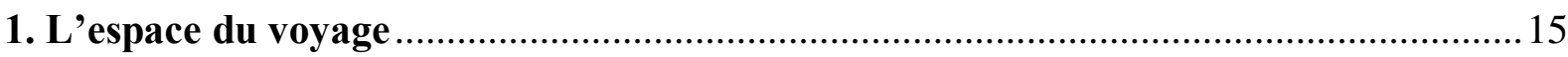

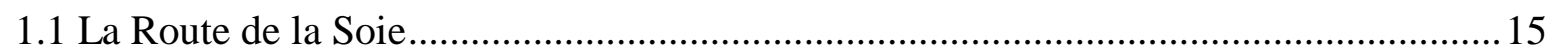

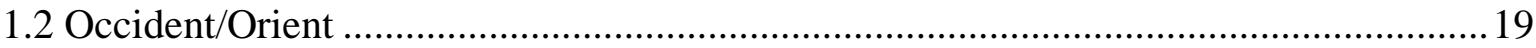

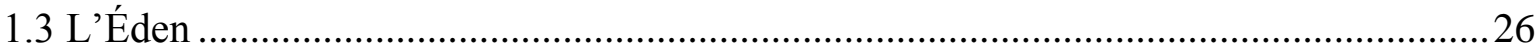

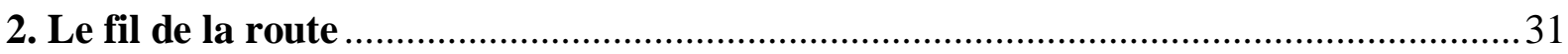

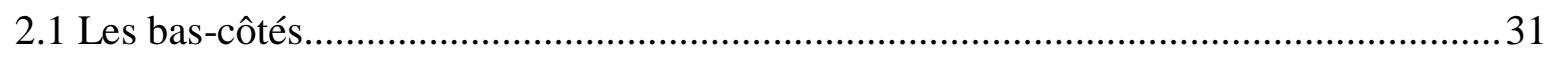

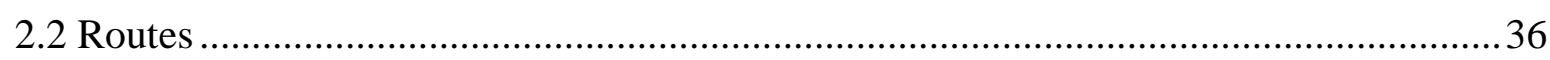

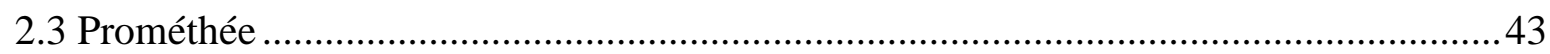

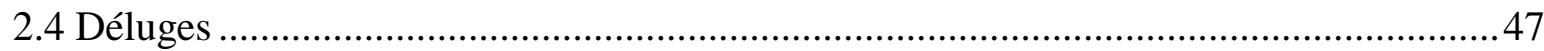

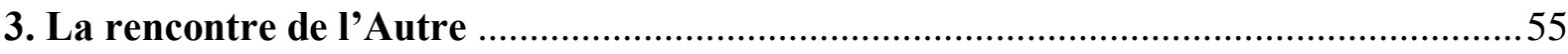

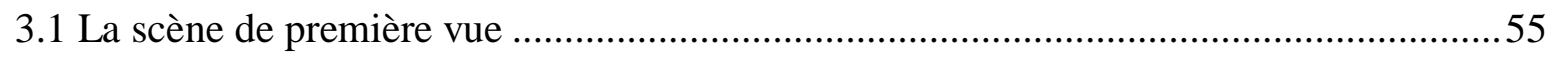

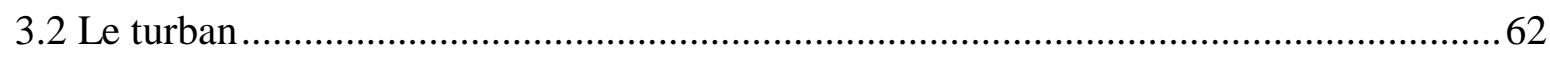

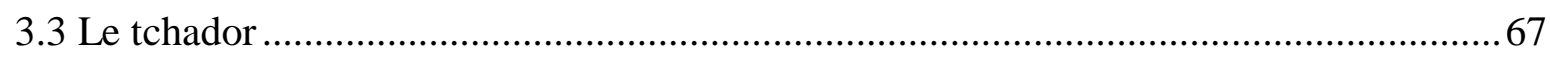

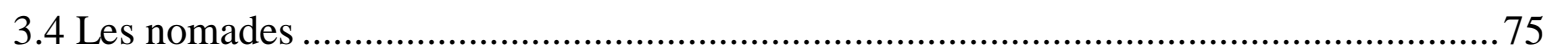

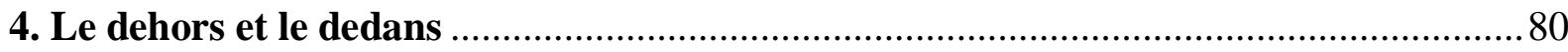

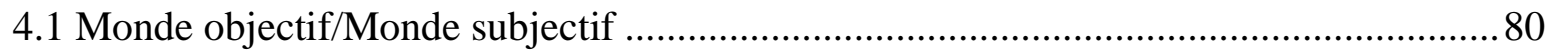

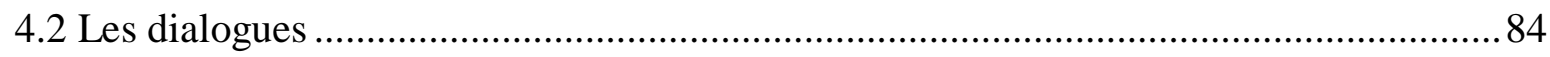

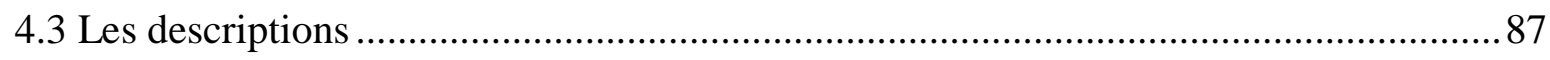

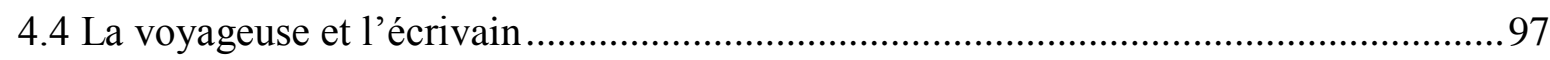

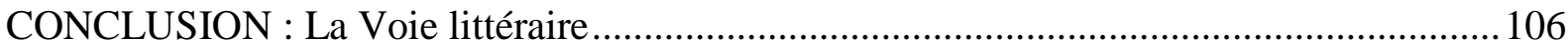

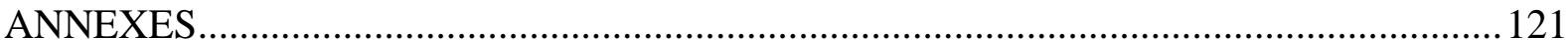

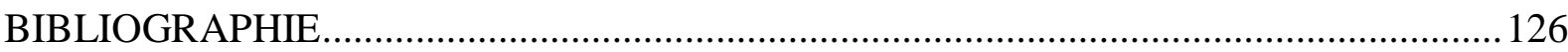




\title{
INTRODUCTION : La « fin des voyages »
}

Alors que les Grandes Découvertes avaient entrainé l'apogée du récit de voyage, on a pu dire que le XXe siècle avait assisté à ce que Claude Lévi-Strauss, dans Tristes Tropiques, appelle «la fin des voyages » et, par conséquent, au progressif tarissement des récits qui en rendent compte.

\begin{abstract}
Je comprends alors la passion, la folie, la duperie des récits de voyage. Ils apportent l'illusion de ce qui n'existe plus et qui devrait être encore, pour que nous échappions à l'accablante évidence que vingt mille ans d'histoire sont joués. Il n'y a plus rien à faire : la civilisation n'est plus cette fleur fragile qu'on préservait, qu'on développait à grand-peine dans quelques coins abrités d'un terroir riche en espèces rustiques, menaçantes sans doute par leur vivacité, mais qui permettaient aussi de varier et de revigorer les semis. L'humanité s'installe dans la monoculture ; elle s'apprête à produire la civilisation en masse, comme la betterave. Son ordinaire ne comportera plus que ce plat. (LÉVI-STRAUSS, 1955 : 36-37)
\end{abstract}

Les voyages, après avoir ouvert l'Occident à la variété des sociétés humaines, ont fini par répandre dans leur sillage une seule et même culture. La « civilisation », «fleur fragile » et menacée - mais aussi revigorée - par les « espèces rustiques » qui l'environnaient, occupe désormais toute la place. La «monoculture » a finalement eu raison de la diversité culturelle. Le monde, autrefois plein de mets variés, ne sert plus au voyageur, où qu'il aille, qu'un seul et même « plat ».

Avec la variété du monde, les voyages ont pris fin eux aussi. Au XVIe siècle, en mettant le pied au Brésil, Jean de Léry pouvait avoir le sentiment d'aborder un autre monde ; les différences étaient si grandes, entre un continent et l'autre, que la traversée relevait alors vraiment du voyage. Aujourd'hui, l'avion nous emmène plus loin, et plus vite, que les galions et les caravelles, mais on ne sort plus vraiment de chez soi, on ne voyage plus ; on se déplace seulement d'un bout à l'autre du même monde.

Pourtant, il semble que la civilisation exige de ses voyageurs qu'ils maintiennent «l'illusion de ce qui n'existe plus », qu'ils témoignent encore de cette variété qu'elle a ellemême étouffée. Menacée par sa propre «fadeur», la société occidentale attend encore de ses voyageurs, autrefois pourvoyeurs de grains de poivre et d'autres épices, qu'ils continuent 
d'apporter, mais «cette fois sous forme de photographies, de livres et de récits, les épices morales dont notre société éprouve un besoin plus aigu en se sentant sombrer dans l'ennui ». Cependant,

ces modernes assaisonnements sont, qu'on le veuille ou non, falsifiés. Non certes parce que leur nature est purement psychologique ; mais parce que si honnête que soit le narrateur, il ne peut pas, il ne peut plus nous les livrer sous une forme authentique. Pour que nous consentions à les recevoir, il faut, par une manipulation qui chez les plus sincères est seulement inconsciente, trier et tamiser les souvenirs et substituer le poncif au vécu. (LÉVISTRAUSS, $1955: 37$ )

Alors qu'au XVIe siècle, les voyageurs avaient à déployer toute une batterie de procédés pour convaincre leurs lecteurs de la véracité de leurs aventures - avec d'autant plus d'insistance que les affabulations de confrères moins épris de vérité avaient pu jeter la suspicion sur leur profession -, au XXe siècle, c'est par d'autres procédés, et en particulier l'occultation, le tri, la «manipulation », que les voyageurs veulent persuader un public consentant que le monde n'a pas changé : que celui que décrivait, par exemple, Jean de Léry, existe encore dans les forêts brésiliennes. D' «histoire vraie » cherchant à convaincre un public incrédule de ce qui existait, le récit de voyage ne serait plus qu'une «duperie», destinée à maintenir un public d'une «crédulité naïve » dans l'illusion de l'exotisme et du voyage (LÉVI-STRAUSS, 1955 : 38). De témoignage, le récit ne serait plus qu'une fable.

Aussi, le récit de voyage, au XXe siècle, aurait été la survivance d'une pratique anachronique : développé au XVIe siècle, dans un monde dont la variété justifiait qu'on en rendît compte, il aurait dû disparaître avec l'expansion de la « civilisation » et l'absorption de la diversité culturelle dans la «monoculture » planétaire. Cependant, alors que le monde des voyages a disparu, la littérature de voyage aurait continué d'exister, comme le rayonnement d'une étoile persiste encore longtemps après que cette dernière a disparu.

Après la fin des voyages, cependant, les récits de voyage auraient fini, eux aussi, par tarir. De fait, il semble bien que la littérature de voyage, au XXe siècle, se soit un temps éclipsée. Le devenir des textes d'Ella Maillart, dernière héritière d'une tradition du voyage en Orient qui remonte à Marco Polo, peut se montrer éclairant.

L'essentiel de son œuvre, publié entre 1932 et 1947, relate des voyages effectués dans les années 30. Par la suite, et même si Ella Maillart ne s'éteindra qu'en 1997, elle n'écrira plus de récit de voyage et, pendant longtemps, ses livres ne seront même plus réédités. En 
effet, comme le constate Nicolas Bouvier en 1971, un texte comme Oasis interdites dont il salue la réédition tardive, était « introuvable depuis vingt ans ». Le silence d'Ella Maillart, le tarissement de ses livres - et même les difficultés rencontrées par Nicolas Bouvier, en 1963, pour publier L'Usage du monde - tendent à montrer le désintérêt des éditeurs et des lecteurs pour la littérature de voyage. De l'après-guerre, jusqu'au début des années 1970, il y a, comme le souligne Charles Forsdick dans son essai sur Oasis interdites, « une lacune évidente dans l'évolution du récit de voyage, qui coïncide avec ce que Claude Lévi-Strauss nomme dans Tristes Tropiques l'angoisse de la "fin des voyages". »(FORDICK, 2008 : 12). La fin des voyages aurait donc eu pour conséquence ultime la fin des récits de voyages.

Néanmoins, il semble que ces récits aient bénéficié d'un regain d'intérêt dans les années soixante-dix comme en témoignent la réédition, en 1971, d'Oasis interdites, et la traduction tardive de deux autres textes d'Ella Maillart initialement écrits en anglais : Ti-Puss (1951), publié pour la première fois en français en 1979, et Gypsy Afloat (1942), traduit sous le titre La Vagabonde des mers en 1991.

Le renouvellement du genre dépendait en partie de la reconnaissance de nouvelles voix dans ce domaine: Bouvier et Le Bris eux-mêmes, mais également Jacques Lacarrière, Jacques Meunier et Kenneth White, un groupe d'écrivains qui ont essayé, séparément d'abord, de découvrir « une littérature qui dise le monde », qui arriverait à décrire le monde dans toute sa complexité. Comme en témoigne la bibliographie donnée à la fin de Pour une littérature voyageuse, la découverte de cette nouvelle tendance littéraire s'est accompagnée de la redécouverte de prédécesseurs, de figures de proue dont l'œuvre avait contribué à la formation de la nouvelle génération. Victor Segalen y a joué un rôle majeur, mais l'importance de Maillart a été reconnue, sans doute en partie grâce à la médiation de Nicolas Bouvier. (FORSDICK, $2008:$ 13)

Aussi, l'édition des livres d'Ella Maillart suit-elle l'histoire du récit de voyage au XXe siècle : de dernière héritière d'une littérature apparemment condamnée à disparaître avec le monde qui lui avait donné naissance, elle se trouve récupérée, une vingtaine d'années plus tard, par une nouvelle génération d'auteurs qui la mettent au rang de ses « prédécesseurs », de ses «figures de proue ». Curieux destin qui, du vivant de son auteur, fait d'une même œuvre le dernier rayon d'une époque et la première lueur d'une autre. Les livres d'Ella Maillart marqueraient à la fois le crépuscule et la renaissance du récit de voyage, séparés l'un de l'autre par une nuit de vingt années. 
Est-ce à dire que Claude Lévi-Strauss s'était trompé et que le monde méritait encore une littérature qui sache le dire ? L'auteur de Tristes Tropiques s'est-il montré trop pessimiste quand il annonçait la «fin des voyages » et dénonçait la «duperie des récits de voyages »? Sans doute sa vision mérite-t-elle d'être nuancée, comme le rappelle Olivier Hambursin dans son «Préambule à quelques promenades au cœur du XXe siècle», en apportant «deux amendements à ces constats pessimistes » :

La première touche à l'uniformisation du monde. Rien ne prouve qu'elle soit, en effet, si universelle que certains le laissent entendre [...] Par ailleurs, le fait de proclamer épuisé un genre, sous prétexte que les différences s'estomperaient entre les objets qu'il décrit, néglige d'autres dimensions essentielles du récit de voyage, à savoir l'écriture, la dimension littéraire, le plaisir du texte qui crée ou recrée sans cesse le monde. (HAMBURSIN, $2005: 10$ )

Le monde, sans aucun doute, a vu ses différences s'estomper peu à peu, en partie, d'ailleurs, à cause de ces mêmes voyageurs qui, au long des siècles, en ont décrit la diversité. Cependant, si l'objet que cette littérature s'était donnée pour fonction de décrire a perdu peu à peu de son intérêt, ce n'est pas tant la littérature de voyage qui s'en est trouvé menacée, qu'une certaine manière de concevoir la relation de voyage. Et si la nécessité de rendre compte du monde par une écriture brute, transparente, sans prétention littéraire, a pu s'imposer quand il était nécessaire de le décrire, de porter à la connaissance de tous la grande variété de ses paysages et de ses peuples, l'effacement progressif de cette diversité a pu, en retour, conduire les auteurs à valoriser d'autres aspects du genre : non plus ceux liés à son objet, mais ceux qui relèvent de son langage. Le travail de l'écriture, «la dimension littéraire », aujourd'hui que le monde n'est plus à découvrir, a fini par prendre toute son importance. Car sans doute, une écriture qui décrit le monde n'est plus aussi nécessaire qu'une écriture «qui crée ou recrée sans cesse le monde ». Et le «plaisir du texte » de voyage ne provient plus tellement de ce qu'il montre, mais peut-être bien de la façon dont il s'écrit.

Peut-être aura-t-il fallu vingt années de gestation, entre le dernier récit d'Ella Maillart, dans les années cinquante, et la réédition de ses livres, dans les années soixante-dix, pour que s'opère cette transformation, et que les voyageurs inventent une nouvelle écriture, imposant pleinement le récit de voyage comme genre littéraire.

Si Ella Maillart représente un chaînon essentiel dans la littérature de voyage du XXe siècle, La Voie cruelle marque un tournant au cœur de son œuvre. De 1930 à 1940, Ella 
Maillart réalise cinq grands voyages en Asie, dont quatre se transforment en récits. Or, si chaque livre relate un itinéraire précis, et peut se lire indépendamment des autres, chacun compose néanmoins un chapitre dans l'ensemble de l'œuvre et une étape dans une recherche qui débute avec le premier et trouve son aboutissement dans le dernier.

On peut diviser l'œuvre d'Ella Maillart en trois ensembles. Le premier se compose de ses trois premiers récits de voyages : Parmi la jeunesse russe (1932), Des Monts célestes au sables rouges (1934), et Oasis interdites (1937). Chacun de ces livres s'inscrit en effet très clairement dans le prolongement du précédent. Ainsi, le voyage relaté dans le deuxième récit est non seulement financé par la publication du premier, mais revient en Russie où Ella Maillart peut satisfaire sa curiosité pour la région du Turkestan. Or, c'est au cours de ce second voyage qu'elle entrevoit, du haut des T'ien Chan (les «Monts célestes »), sur la frontière entre la Russie et la Chine, le désert du Takla-makan et la province du Sin-kiang où la conduira, depuis Pékin, le voyage relaté dans son troisième récit, Oasis interdites.

Aussi, non seulement chaque récit prolonge le second, mais l'ensemble définit peu à peu un point de convergence aux itinéraires d'Ella Maillart (voir annexes 1a et 1b) qui tous, à partir de l'Est tout d'abord, puis de l'Ouest, tendent vers cette région centrale de l'Asie qu'on appelle les Pamirs.

\footnotetext{
Nous avancions sur un «pamir» où les territoires des Soviets de l'Afghanistan, de l'Inde et du Sin-kiang sont à quelques kilomètres les uns des autres : politiquement et géographiquement, c'est un des «nœuds» de l'Asie. Les Pamirs, «le toit du monde », ne sont pas plats comme je l'avais vaguement imaginé dans mon enfance. L'expression « toit» provient du fait qu'aucune des vallées de cette région n'est située au-dessous de trois mille mètres et que les chaînes qui constituent les grandes arêtes de l'Asie : les T'ien-chan, l'Hindou Kouch, les Himalayas, et le Kouen-louen, semblent toutes rayonner de ce même point de départ. (MAILLART, 2001b : 198)
}

Les Pamirs sont donc un «nœud» de l'Asie, pour des raisons tout à la fois politiques - les frontières de trois pays «sont à quelques kilomètres » les unes des autres - que géologiques : c'est le lieu d'où les grandes chaînes de montagnes du continent «semblent toutes rayonner ». Or, c'est vers ce centre névralgique de l'Asie que tendent tous les itinéraires d'Ella Maillart, et vers lequel la ramène une quatrième fois celui qu'elle relate dans La Voie cruelle, comme si voyager, c'était vouloir atteindre « ce point de départ » : comme si voyager, c'était vouloir atteindre un point d'origine.

Le deuxième ensemble de textes est composé de deux livres écrits en Inde, pendant la Seconde Guerre Mondiale, et publiés en 1942. Il s'agit, d'une part, de Gypsy Afloat, qui ne 
sera traduit en français sous le titre La Vagabonde des mers qu'en 1991 et, d'autre part, de Cruises and Caravans, traduit sous le titre Croisières et caravanes en 1951. Ces textes se distinguent des trois précédents en ce qu'ils constituent une autobiographie d'Ella Maillart en deux volumes : le premier relate les navigations de son «insouciante jeunesse », dans les années 20, «elle-même inscrite dans un infime et insouciant moment de l'existence des hommes » (MAILLART, 1991: 11) ; le second volume revient lui aussi sur ces expériences de mer, auxquelles il ajoute le récit de ses exploits sportifs - Ella Maillart fut championne de ski - et surtout de ses voyages dans l'Asie des années 30. C'est dans ces livres, et surtout le deuxième que, revenant sur ses périples tant sur mer que sur terre, Ella Maillart en perçoit la cohérence. Car si elle peut écrire à trente-neuf ans son autobiographie, c'est qu'elle vient de refermer un cycle et, surtout, de trouver en Inde, dans l'enseignement de deux maîtres spirituels, ce qu'elle avait cherché tout au long de ses voyages. «Aux Indes, j'étais au début d'un voyage tout nouveau qui devait me conduire plus avant vers la vie complète et harmonieuse que je cherchais instinctivement» (MAILLART, 2001b: 224). Aussi, l'autobiographie d'Ella Maillart montre-t-elle comment ses voyages géographiques trouvent leur aboutissement dans un voyage spirituel, dont le centre n'est plus situé dans la région asiatique des Pamirs, mais au cœur même de la voyageuse.

Le troisième ensemble de textes est composé par The Cruel Way (1947), traduit en français par Ella Maillart sous le titre La Voie cruelle en 1951, et Ti-Puss (1951) qui ne sera traduit en français qu'en 1979. Ces deux récits sont eux aussi écrits en Inde, peu après les deux volumes autobiographiques. Dans La Voie cruelle, Ella Maillart relate son quatrième et dernier grand voyage des années 30, entre Genève et Kaboul ; cependant, entre le voyage et son écriture, trois années se sont écoulées, et son expérience indienne imprègne ce récit de voyage qui marque dans son œuvre une transition entre le voyage dans le monde réel et le voyage dans le monde subjectif. Ti-Puss est quant à lui un livre assez étonnant où le voyage cède le devant de la scène au personnage éponyme. En effet, c'est en observant Ti-Puss diminutif de «petit pussy », nom qu'Ella Maillart donne à la chatte qui l'accompagne au long de son séjour - et non pas en observant l'Inde, qu'elle découvre cette présence au monde dont les animaux sont naturellement dotés, et que visent à lui enseigner les maîtres spirituels. Plus tard, en 1951, Ella Maillart publie un reportage intitulé The Land of the Sherpas, qui porte sur ses voyages au Népal, et qui n'est toujours pas traduit en français ; cependant, on peut considérer qu'avec Ti-Puss, l'œuvre d'Ella Maillart est close : c'est dans ce livre, en effet, qu'elle atteint son but et donne aux autres récits une cohérence et un sens rétrospectifs. Et 
même si Ella Maillart vit jusqu'en 1997, et fait de nombreux autres voyages en Asie, elle ne publie aucun autre livre, consciente, sans doute, du caractère abouti de son œuvre.

La Voie cruelle occupe donc une situation intermédiaire dans l'œuvre d'Ella Maillart : placé entre Oasis interdites et Ti-Puss - si l'on écarte les deux textes autobiographiques - ce récit marque un passage entre voyage géographique et quête spirituelle, entre récit d'exploration et récit d'introspection.

L’itinéraire géographique de ce récit peut être découpé en trois grandes étapes : de la Suisse à la Turquie (chapitres 1 à 9) ; l'Iran (chapitres 10 à 18); et, finalement, l'Afghanistan (chapitres 19 à 29); à quoi s'ajoute un épilogue en Inde (chapitre 30).

Cependant, dès le départ, ce voyage double son itinéraire d'une trajectoire intérieure. En effet, Ella Maillart prétend non seulement atteindre l'Afghanistan et, au-delà, la région des Pamirs, mais elle espère aussi aider sa compagne de voyage, Christina, à guérir de sa dépression et de sa dépendance à la morphine. «Au fond de moi-même, j’étais convaincue d'atteindre mon double but, soit aider mon amie et arriver à Kaboul » (p. 32). Cette double dimension du voyage s'incarne ainsi dans le tandem que forment la voyageuse et l'écrivain, «Kini » - surnom d'Ella Maillart - et «Christina - pseudonyme d'Annemarie Schwarzenbach, journaliste et écrivain suisse de langue allemande (voir annexe 2). Mais elle s'incarne aussi dans la personne même de Christina : en effet, c'est elle qui fournit avec la Ford un moyen de transport aux deux voyageuses, et dont les tourments intérieurs confèrent aussi à l'aventure son enjeu psychologique.

On retrouve enfin ce double aspect dans le titre même du récit, La Voie cruelle pouvant non seulement renvoyer aux difficultés du voyage, mais aussi - et surtout - à «la voie compliquée, la voie cruelle de l'enfer» (p. 125) suivie par Christina, dont le décès accidentel, en 1943, incite Ella Maillart à écrire le récit de leur voyage.

On s'efforcera donc, dans cette étude, de montrer le caractère éminemment transitoire de La Voie cruelle. Car s'il témoigne des bouleversements du XXe siècle qui, à la même époque, amènent Claude Lévi-Strauss à annoncer la «fin des voyages », le livre marque aussi un tournant dans l'œuvre d'Ella Maillart.

Ainsi, dans la première partie, on montrera tout d'abord combien ce récit s'inscrit dans la grande tradition des voyages en Orient, en prenant d'une part un itinéraire qui suit la Route de la Soie, et perpétuant d'autre part une représentation de l'espace asiatique héritée de l'époque médiévale. Cependant, dans les deuxième et troisième chapitres, les études du voyage et de la rencontre de l'Autre révèleront combien, au XXe siècle, l'expansion vers l'Est 
d'une seule et même civilisation tend à uniformiser les paysages et ceux qui les habitent, remettant en question l'utilité des voyages fondés sur la découverte des différences. Ainsi, l'ensemble de ces trois chapitres s'efforcera de montrer en quoi, d'une part, l'héritage du passé et, d'autre part, l'évolution du XXe siècle, fond du récit d'Ella Maillart le prolongement d'un genre qui remonte au Moyen-Âge, et probablement aussi, l'un de ses derniers héritiers.

Enfin, dans le quatrième chapitre, on reviendra sur la place occupée par La Voie cruelle dans l'œuvre d'Ella Maillart, tout à la fois adieu au voyage géographique et prémisse d'une quête intérieure, et l'on montrera combien ce passage, influencé par l'exemple d'Annemarie Schwarzenbach, ouvre pour le récit de voyage une voie vers l'écriture littéraire. 


\section{LA VOIE CRUELLE (1952) D'ELLA MAILLART}

\section{L'espace du voyage}

L'itinéraire suivi par Ella Maillart dans La Voie cruelle s'inscrit dans un des espaces les moins vierges qui soient pour un voyageur : l'Orient. En effet, non seulement il s'agit d'un espace où, depuis l'Antiquité, d'autres ont inscrit leurs propres sillages, mais surtout, il s'agit du continent sur lequel on compte le plus grand nombre de témoignages : plus qu'un espace déjà parcouru, il s'agit avant tout d'un espace déjà écrit. Or, qui s'aventure en Asie ne peut ignorer cet héritage, ni se soustraire aux représentations qui, au long des siècles, ont structuré le continent et donné au voyage en Orient d'autres dimensions que celles d'un simple déplacement géographique.

\subsection{La Route de la Soie}

Ella Maillart s'inscrit dans la grande tradition des relations de voyage en choisissant, tout d'abord, un itinéraire qui suit «l'une des plus anciennes voies commerciales dont nous connaissions l'histoire : la transasiatique Route de la Soie» (p. 80). Aussi, reprendre cette route, c'est non seulement traverser l'espace qui sépare l'Europe de l'Asie, c'est aussi revenir sur les traces d'innombrables voyageurs. Et, en mettant le pied sur cette route, à Trébizonde, il semble qu'Ella Maillart se fonde dans « la multitude de guerriers, de rois, de voyageurs, de missionnaires, de marchands, d'ambassadeurs et d'hommes de lettres qui la suivirent » (p. 80), à commencer par le plus illustre d'entre eux, Marco Polo.

Ella Maillart revient en effet sur les pas du voyageur vénitien à plusieurs reprises : à Trébizonde (p. 80), à Erzeroum (p. 89), à Tabriz (p. 109), à Balkh (p. 234); et ce n'est pas le seul voyageur dont elle croise l'itinéraire : elle revient aussi sur les pas de Tavernier (p. 134), de l'empereur Babur (p. 199), du pèlerin Hiuan Tsang (p. 234, p. 266), ou encore d'Ibn Battuta (p. 254). Où qu'on aille en Asie, plus qu'en aucun autre continent, il est rare d'être le premier voyageur. 
Ella Maillart ne se contente pas d'évoquer le nom de ceux qui, au long des siècles, l'ont précédée ; elle cite aussi leurs textes, dont elle reproduit même de longs passages ${ }^{1}$, qu'il s'agisse de Marco Polo décrivant «la grande et très noble cité de Tabriz » (p. 109), d'un « ambassadeur chinois » décrivant la ville d'Hérat au XVe siècle (p. 197), ou encore de Babur évoquant le Nidjrau (p. 289). C'est qu'Ella Maillart ne marche pas seulement dans les pas de ses prédécesseurs : elle marche aussi dans leurs livres. Comme l'affirme Gilles Lapouge, «tout voyage, y compris dans les terres inconnues, n'est que le souvenir d'une encre ancienne. C'est ce qui rend déprimante la profession d'explorateur : vous ne marchez jamais que dans les encres des explorateurs qui vous ont précédé » (LAPOUGE, 2007 : 9). Si la remarque de Gilles Lapouge s'applique même à des «terres inconnues » - Christophe Colomb ayant moins découvert l'Amérique, qu'il n'a cru aborder les terres décrites par Marco Polo - a fortiori, elle peut s'appliquer à un continent aussi connu que l'Asie.

L'Asie est un continent de l'écrit : non seulement d'innombrables voyageurs l'ont déjà décrite, mais son histoire millénaire, relatée par les historiens de l'Antiquité - tel Xénophon relatant l'expédition des Dix Mille (p. 82-83) - remonte même jusqu'à la Bible - en particulier, on le verra, à l'histoire du déluge - et à d'autres textes fondateurs. Dès lors, voyager sur les routes d'Asie revient à circuler dans une immense bibliothèque ; et écrire sur l'Asie, revient à placer sa propre écriture au cœur d'un immense réseau d'intertextualités ${ }^{2}$.

Les deux voyageuses transportent d'ailleurs avec elle une vraie bibliothèque :

Nous voyagions avec une rangée de livres fixés au-dessus de notre dossier. Les pauvres volumes étaient terriblement secoués, mais c'était une joie de pouvoir mettre la main sur l'ouvrage désiré au moment voulu. Cahotant l'un contre l'autre, il y avait là Marco Polo, Paul Pelliot, Evans-Wentz, Vivekananda, Maritain, Jung, une vie d'Alexandre le Grand, René Grousset, le Zend-Avesta. (p. 237)

Le monde qui entoure Ella Maillart, tant de fois décrits par d'autres, est tout autant à lire qu'à voir : dès qu'elles abordent l'Orient, les deux voyageuses n'ont-elles pas

\footnotetext{
${ }^{1}$ L'édition originale de La Voie cruelle (Genève : Jeheber, 1952) contient à la page 231 un Index des œuvres citées par Ella Maillart dans le récit.

${ }^{2}$ La Voie cruelle entre à son tour dans le corpus des textes sur l'Asie quand d'autres voyageurs, tels Nicolas Bouvier, reprennent quelques années plus tard la même route qu'Ella Maillart : «J'ai rencontré Ella Maillart en 1952 pour lui demander des avis sur la route Genève-Madras qu'elle avait faite à deux reprises et que nous comptions, un ami et moi, emprunter. Ses conseils furent d'une sobriété toute britannique : "Partout où des hommes vivent, un voyageur peut vivre aussi..." » (MAILLART, 1991 : 7)
} 
l'impression, en approchant d'Edirne, de contempler, « éclairée par les rayons horizontaux du soleil couchant, une vision des Mille et Une Nuits » (p. 60) ? Ella Maillart ne parle-t-elle pas de Hiuan Tsang comme du «pèlerin dont j'ai croisé les traces au cours de trois voyages en Asie centrale et dont les écrits m'ont tant aidé à apprécier ce que j’y voyais » (p. 234) ? Aussi, la citation matérialise-t-elle dans le récit l'interposition d'un texte ancien entre la voyageuse et le monde : elle perçoit les paysages au travers d'un texte - on pourrait même avancer que la citation matérialise la superposition, voire même la substitution, d'un paysage $l u$ au paysage $v u$.

La plupart des œuvres auxquelles Ella Maillart fait référence remontent à l'Antiquité ou au Moyen-Âge. Aussi, la citation permet-elle de mesurer à l'aune des textes anciens les bouleversements imposés par l'histoire aux pays traversés ou, au contraire, d'attester leur résistance au passage du temps. Mais il semble bien qu'Ella Maillart ne soit jamais aussi satisfaite que lorsque le paysage lu dans un texte antique se superpose exactement au paysage vu lors du voyage, comme si, plutôt que de vouloir mesurer les changements intervenus, elle voulait plutôt vérifier l'immutabilité des choses et des hommes. Ainsi, à propos de Badakhchan, Ella Maillart relève que « Hiuan Tsang écrivit aussi que "la plupart des habitants ont les yeux gris-bleu et par là se distinguent des peuples voisins", ce qui est encore vrai aujourd'hui » (p. 241). La voyageuse tire plaisir de confirmer par ses propres yeux une information qu'elle a déjà lu. Comme si le voyage en Asie ne pouvait se dissocier d'une volonté de remonter dans le temps, de retrouver un état plus ancien du monde, plus originel. Et la citation, dispensant la voyageuse d'écrire sa propre description d'un lieu, manifeste ainsi la satisfaction de n'avoir pas à ajouter une ligne à ce qui a déjà été dit et se révèle toujours d'actualité.

Le bonheur de la citation, qui révèle la nature d'un voyage cherchant moins le nouveau que l'ancien, le changement que la permanence, n'exclut pas tout à fait, cependant, le bonheur de la découverte, aussi rare puisse-t-il être sur un continent comme l'Asie. Ainsi, près de la Caspienne, les voyageuses ont le plaisir de découvrir une mosquée. «Il est possible que cette mosquée aux apparences d'église ne soit pas un monument historique. Mais nous l'aimions parce qu'elle était notre découverte : aucun sentiment de devoir envers un Baedeker ne nous avait poussées à la visiter ! (p. 149). Cependant, il s'agit du plaisir de découvrir un monument dont personne n'a encore parlé, que les guides de voyage, tels que le Baedeker, n'ont pas encore recensé : le plaisir vient ici de combler une lacune, non pas de corriger ce qui est déjà écrit. 
De plus, Ella Maillart ne confronte pas seulement ce qu'elle voit à ce que d'autres, bien avant elle, ont déjà décrit : elle compare aussi les lieux qu'elle traverse aux souvenirs que lui en ont laissés ses propres voyages. En effet, ce n'est pas la première fois qu'Ella Maillart emprunte cette route. «C'était la cinquième fois que je partais pour l'Asie. Je connaissais le chemin jusqu'à Herat en Afghanistan, en sorte que, jusque là, je ne pouvais pas tomber sous le charme excitant de la découverte » (p. 58). Et si, jusqu'au bout de son itinéraire, Ella Maillart ne découvre rien que d'autres voyageurs, avant elle, n'aient déjà découvert, jusqu'en Afghanistan, elle ne découvre rien non plus qu'elle n'ait déjà vu de ses propres yeux. Aussi, Ella Maillart revient-elle, durant deux tiers du récit, sur ses propres traces, que ce soit près de Milan (p. 38), à Makou (p. 103), à Chalus (p. 120), à Mechhed (p. 170), ou encore à Islam Kaleh (p. 187). Il lui faudra attendre de dépasser Herat pour que le voyage puisse au moins lui procurer «cette attente impatiente, cette constante excitation éveillée par l'inconnu » (p. 205), et qu'elle retrouve une sensation de « découverte », sinon à l'échelle de l'histoire humaine, du moins, au niveau de son histoire personnel.

Ainsi Ella Maillart éprouve-t-elle doublement ce que François Moureau appelle une sensation de «déjà-vu »: d'une part, en ce qu'elle traverse des lieux qu'elle a tout d'abord lu avant de les voir - le déjà-vu se confondant alors avec un déjà-lu ; d'autre part, en ce qu'elle en est à son «second voyage » sur une même route. « Nous voyageons avec notre mémoire et notre culture » (MOUREAU, 1996 : VII).

De plus, de même que le récit redouble l'impression de déjà-vu, il redouble aussi l'impression d'un déjà-écrit : en effet, non seulement Ella Maillart cite les témoignages que d'autres ont laissés sur l'Asie, mais elle-même a déjà écrit sur ce continent - tous ses récits de voyages prennent l'Asie pour espace - et surtout sur ce même voyage. En effet, La Voie cruelle n'est pas la seule relation qu'Ella Maillart ait tirée de son voyage en Afghanistan avec Annemarie Schwarzenbach. D'une part, à l'intérieur même du récit, elle représente les deux voyageuses rédigeant des articles pour une agence dont elles sont les correspondantes: «Ayant adopté des expressions sérieuses et nous grattant parfois la tête, nous avions commencé à écrire nos lettres de voyage promises à une agence de presse » (p. 73); et Ella Maillart cite même un de ses propres articles sur «les chauffeurs de l'Iran, héros de l'Iran moderne » (p. 112). À ces articles s'ajoute d'autre part le courrier écrit en chemin : le recueil Cette réalité que j'ai pourchassée nous livre deux lettres envoyées par Ella Maillart à sa mère depuis l'Afghanistan (MAILLART, 2008 : 134 et 137). Surtout, elle raconte une première fois ce même voyage, en 1942, dans la brève relation qu'elle en fait au chapitre XIII de Croisières 
et caravanes, intitulé «Vers l'Afghanistan». Enfin, La Voie cruelle est un récit qu'Ella Maillart écrit deux fois : une première fois en anglais, une seconde fois, en français.

Cette réécriture d'un même voyage sous différentes formes est une dimension propre aux récits de voyage, - ce que Charles Forsdick, à propos d'un autre texte d'Ella Maillart, appelle « la polygraphie » :

Cette dimension du genre se voit dans les différentes versions du voyage produites sur le terrain même, dans les carnets de route, les photographies qui l'accompagnent, les lettres à sa mère, les articles pour Le Petit Parisien, les conférences faites à son retour en Suisse, en France et en GrandeBretagne, et bien sûr dans le récit de voyage lui-même. (FORSDICK, 2008 : 92)

Ainsi La Voie cruelle ajoute à l'encre d'autres voyageurs ayant parcouru la Route de la Soie, et au déjà-vu d'un itinéraire qu'Ella Maillart a elle-même suivi plusieurs fois, la polygraphie propre à un genre dont les auteurs racontent un même voyage sous des formes très diverses.

Par conséquent, encore moins que tout autre récit asiatique, La Voie cruelle ne prétend faire découvrir à son lecteur un continent inconnu, pas plus qu'il ne se présente comme un récit inédit. Au contraire, par de multiples renvois sous formes d'allusions ou de citations, $L a$ Voie cruelle revendique une intertextualité riche et multiple qui place le récit dans un réseau de représentations héritées de la tradition des voyages en Orient.

\subsection{Occident/Orient}

L'Occident et l'Orient divisent l'espace du voyage non pas seulement comme deux moitiés géographiques, mais plutôt comme deux pôles opposés. L'Europe menacée par la guerre constitue dans l'espace du récit un pôle répulsif, et l'Asie qui, au contraire, en est préservée, un pôle attractif. Le début du récit pose d'ailleurs clairement les termes en opposant un Ici intolérable, menacé par une guerre imminente, à un Ailleurs idéalisé.

Nous sommes à l'hiver 1938, Christina vient d'évoquer l'annexion des Sudètes et les menaces que fait peser l'armée allemande sur Prague : «elle venait de décrire l'âme de cette ville, la vie de ses amis tchèques, leur impuissance et leur désespoir devant l'emprise d'un ennemi chaque jour plus proche, plus implacable » (p. 21). Le décor est planté : l'Europe à la veille de la seconde guerre mondiale, et les concessions des démocraties aux exigences de 
plus en plus exorbitantes de l'Allemagne, qui servent moins à prolonger la paix qu'à retarder la guerre. Et, regardant alors par la fenêtre, c'est leurs sombres pensées que les deux amies, Christina et Kini, projettent sur le paysage alpin.

Un ciel bas et sombre pesait sur la vallée livide, sans ombre et comme morte. Quoique dans les hautes Alpes, la contrée semblait plate et vaste car la maison était au bord d'un lac gelé recouvert de nombreuses couches de neiges. Il n'y avait que cette austère désolation entre nous et l'horizon où le col de la Maloja mène en Italie. (p. 21)

Le ciel pèse sur la vallée comme la menace de guerre sur l'Europe. Le poids de cette menace est même si lourd que, bien qu'on soit en montagne, «la contrée semblait plate », écrasée sous «de nombreuses couches de neiges ». Et, quoique la guerre n'ait pas encore éclaté, on dirait qu'elle a déjà tué le paysage : «livide» et «sans ombre », la vallée fantomatique semble bel et bien «morte ».

Cependant, au milieu de cette «austère désolation », le «col de la Maloja » semble encore ouvrir une brèche que le mot «Ford», prononcé par Christina - la voiture dans laquelle les deux amies vont voyager - va permettre d'élargir comme un sésame.

Dans les nuages au dessus de la Maloja, une clarté diffuse semblait indiquer la route; après un plongeon de mille cinq cents mètres dans la chaude Lombardie, elle se faufilerait à travers les Balkans, nous menant jusqu'au Bosphore, porte ouvrant sur les immensités asiatiques. Ma pensée était déjà en Iran. (p. 22)

Il semble que les nuages s'écartent, et qu'une éclaircie pointe, au bout de l'horizon fermé, pour «indiquer la route ». Et s'il s'agit de faire un «plongeon », puis de se faufiler, avant d'atteindre une «porte ouvrant sur les immensités », le départ prend tout d'abord les aspects d'une fuite. Il s'agit moins d'atteindre l'Asie, que de quitter l'Europe - ou plutôt d'échapper à la menace allemande.

Ella Maillart fait alors en «pensée » le voyage et trace l'itinéraire que suivront bientôt les deux amies, de la mer Caspienne aux montagnes de l'Afghanistan (p. 22-23). Cependant, son évocation de l'Asie relève moins d'une description que d'une rêverie, moins d'un voyage réel que d'une évasion. D'une part, en effet, les verbes au futur - «nous visiterons », « nous camperons », « nous verrons », « nous atteindrons », « nous disparaîtrons »- confèrent au texte les accents baudelairiens d'une invitation au voyage. D'autre part, les noms empruntés à la géographie, «Bamiyan », «Band-i-Amir », « Hindou Kouch », « Amou-Daria », quoique bien réels, n’en sont pas moins utilisés ici pour leurs sonorités dépaysantes et leur capacité à 
suggérer l'ailleurs. Et, que ce soit «le dôme en or », «la pure vallée », «les lacs incroyablement bleus ", tout semble paré d'attributs exceptionnels qui confèrent au discours de la voyageuse un caractère moins réel que fabuleux. Il semble qu'elle cherche moins à détailler les étapes d'un parcours, qu'à dépeindre un monde idéal, doré, pur, céleste. Et, de même que le discours de Christina l'avait conduite à Prague «en pensée » (p. 21), Ella Maillart n'en cède pas moins aux vertus hypnotiques de son propre discours : « Ma pensée était déjà en Iran » (p. 22), comme si l'évocation du voyage n'avait eu pour finalité que de proposer à la « pensée » de la voyageuse un idéal capable d'effacer une réalité trop écrasante - un Ailleurs capable d'estomper l'Ici.

Ainsi, le début du récit oppose-t-il d'emblée deux discours: celui de Christina, décrivant l'actualité d'une Europe menacée par la guerre ; celui d'Ella Maillart, qui s'efforce au contraire d'évoquer une Asie idéale. Une Europe historique, d'un côté ; une Asie mythique, de l'autre.

Dans la première partie du voyage, entre le col de la Maloja et le détroit du Bosphore, l'évolution du paysage permet alors de constater avec soulagement l'éloignement de l'Europe, et de mesurer au contraire avec satisfaction l'approche de l'Asie.

Ella Maillart note tout d'abord ce qui inscrit dans le paysage l'emprise du fascisme sur l'Europe et l'imminence de la guerre. Ainsi, « les environs de Trieste étaient défigurés par d'immense DUCE peints sur tous les murs de la route en corniche » (p. 39) ; et plus loin, en Yougoslavie, « le long de la route, les gens nous saluaient souvent à la manière hitlérienne. Un maître d'école leva son bras d'une manière décidée, très exactement imitée par son troupeau d'élèves » (p. 44).

Aussi éprouve-t-elle un soulagement quand, après quelques kilomètres, au sortir de Jagodina, les soldats qu'elle croise n'adressent pas le même salut aux deux voyageuses.

\footnotetext{
À la sortie du village, des soldats faisaient de l'exercice sur la pente d'un coteau. Roulant d'abord au-dessus d'eux puis plus bas qu'eux, nous pûmes admirer leur officier tout en blanc, épée traînant à terre ; de sa langue, il léchait une glace rose dans un cornet. Il nous fit de grands signes de la main. Nous avions enfin dépassé les hommes qui claquent des talons en élevant le bras droit ! (p. 48)
}

Le changement est d'autant plus sensible entre, plus haut, le «troupeau d'élèves » et, ici, cet officier « tout en blanc », que les qualités qui reviendraient normalement aux premiers sont attribuées au second, et vice versa : en effet, ce sont les élèves qui, imitant passivement 
leur maître, adressent aux voyageuses un salut très martial, et c'est l'officier qui, « l'épée traînant à terre » comme s'il abandonnait tout bellicisme, montre une attitude juvénile en léchant «une glace rose dans un cornet». Bien sûr, il s'agit de rendre plus criant l'endoctrinement des enfants en le comparant à la bonhommie du soldat; mais il s'agit aussi, en passant des uns à l'autre, d'éprouver la satisfaction d'avoir « dépassé » les claquements de talons et les bras levés : de constater que la route éloigne peu à peu de l'Europe fasciste, que le voyage en efface petit à petit les signes.

En contrepoint aux marques du fascisme, Ella Maillart relève dès cette première partie du voyage des traits propres à l'Asie. Dès l'Italie, en effet, une procession déploie le long de la route une scène «tout orientale avec ces gens fatigués marchant comme dans un rêve : cela me rappelait des cérémonies semblables vues en Chine ou aux Indes » (p. 38). Plus loin, en Yougoslavie, la voyageuse remarque «des vaches blanches très dignes, aux cornes en forme de lyre », qui lui rappellent « leurs sœurs sacrées des Indes » (p. 45), ou encore des hommes dont les « gilets de mouton brodés paraissaient venir d'Afghanistan » (p. 45).

Et plus tard, quand des centaines de carrioles quittèrent Belgrade, cela aurait pu être les Kazakhs migrateurs d'autrefois quittant Alma-Ata. (Quand, deux ans auparavant, j'arrivai à Belgrade venant d'Asie centrale, mes yeux remarquèrent alors les caractéristiques opposées : cinémas, journaux, barrières, trottoirs, fils électriques...) (p.46)

Ainsi les éléments dont la présence est relevée au fil de la route sont-ils révélateurs du sens dans lequel s'effectue le voyage : au retour d'Orient, les yeux du voyageur noteront la présence de «cinémas, journaux, barrières, trottoirs, fils électriques », tout ce qui inscrit dans le paysage la modernité européenne ; au contraire, au départ de l'Occident, il relèvera ce qui, à la manière des «carrioles », annonce les modes de vie «d'autrefois » de l'Asie. Ainsi, près de Sofia, Ella Maillart note encore que «des hommes portaient déjà des petits turbans et buvaient le café dans des tasses turques » (p. 55).

Par contre, quand elle traverse le Bosphore, ce ne sont plus seulement les premiers signes de l'Orient qu'elle perçoit mais, inversement, les dernières traces de l'Occident :

Avec ses vieux toits aux tuiles bombées, ses ruelles pavées sentant le poisson et ses jardinets emmurés où la rouge fleur du grenadier éclatait devant le bleu de la mer, Trébizonde nichait dans la côte comme une ville italienne exception faite de ses blancs minarets d'où le crieur appelait Allah sous son nom turc de Tanri. (p. 77) 
Et, plus loin, à Gümüshane, ce n'est pas sans nostalgie que la voyageuse suisse relève qu'une

atmosphère de vacances flottait autour des maisons de ce village de montagne ; avec leurs balcons et leurs toits en pente, elles méritaient presque le nom de chalets. C'était là les dernières maisons de ce genre que nous verrions. Ensuite, il n'y aurait plus que des cubes en terre sèche à toit plat. (p. 84)

Sans doute, il s'agit de mesurer dans ces détails du paysage, avec un mélange de satisfaction et de nostalgie, autant l'approche d'une Asie convoitée que l'éloignement progressif d'une Europe rejetée. Cependant, ces rapprochements révèlent aussi des affinités entre des pays et des cultures parfois très éloignés. Et même si Ella Maillart ne prétend pas être «un expert»- «je parle en touriste qui compare des détails vus à Khiva, Boukhara, Samarkand, Herat et Mechhed » (p. 112) ${ }^{3}$-, elle sait néanmoins que certains rapprochements ne sont pas qu'un simple jeu de ressemblances superficielles. Et, quand il s'agit de correspondances entre l'Occident et l'Orient - de même que l'Italie peut rappeler l'Inde ou la Chine, de même, Trébizonde peut évoquer «une ville italienne» (p. 77) $)^{4}$ ces rapprochements révèlent en fait une parenté profonde, une filiation historique. Ella Maillart sait, comme Nicolas Bouvier, «que l'Asie est la mère de l'Europe » (BOUVIER, 2004 : 1214) et qu'il est donc possible de retrouver en Occident, des traits qui rappellent ceux de cette « mère » orientale, et vice versa.

Aussi, l'on peut comprendre la satisfaction qu'éprouve la voyageuse quand, à l'occasion de fouilles en Afghanistan, elle tient entre ses mains les preuves archéologiques d'un lien ancien entre l'Occident et l'Orient :

La qualité tout autant que l'origine de ces pièces vieilles de deux mille ans leur donnait une grande importance. Elles prouvaient que c'était ici, au royaume de Kapisa et non au Gandhara du Haut-Indus, que l'art grec et l'art indien se rencontrèrent, un siècle avant notre ère. La rencontre donna naissance à cet art hybride qui influença le Xinjiang, toute la Chine et le Japon. (p. 290)

3 Chaque voyage constitue peu à peu «un kaléidoscope personnel qui devient notre propre grille de déchiffrement du monde »; ainsi, toutes sortes d' « interférences [...] peuvent jouer selon le parcours personnel de l'écrivain-voyageur ». (MOUREAU, 1996 : VI)

${ }^{4}$ Dans l'édition originale de La Voie cruelle (Genève : Jeheber, 1952), la photographie d'une ville afghane, insérée entre les pages 192 et 193, porte la légende suivante : «Istalif évoque la Grèce ». 
Ces pièces vieilles de deux milles ans sont les traces tangibles d'une continuité historique entre «l'art grec et l'art indien », entre l'Europe et l'Asie - et cette continuité se prolonge même jusqu'en Extrême Orient, puisque de la rencontre de ces arts est issue « cet art hybride qui influença le Xinjiang, toute la Chine et le Japon ». Autrement dit, entre l'Europe et l'Asie, il y a moins une rupture qu'un continuum qui va d'un bout à l'autre des deux continents.

L'Europe et l'Asie sont donc moins opposées qu'une première distinction entre l'Ici et l'Ailleurs pourrait tout d'abord le laisser croire. Malgré les marques du fascisme, il est encore possible de reconnaître des traits qui révèlent une parenté profonde entre les deux continents comme, en creusant la terre, on peut en retrouver les preuves archéologiques.

L'Europe et l'Asie telles que les présente Ella Maillart sont donc tout à la fois opposées et semblables, ce qui caractérise moins un rapport d'opposition que de symétrie : les deux continents se reflètent l'un, l'autre. Cette structure de l'espace géographique en deux moitiés symétriques n'est pas sans rappeler celle que François Hartog met en évidence dans son études des Histoires d'Hérodote.

Hérodote, en composant les Histoires, a utilisé plusieurs cartes et, notamment, une carte ionienne. Or ces cartes font grand usage du principe de symétrie: le monde s'organise symétriquement de part et d'autre d'un « équateur» traversant la Méditerranée [...]. (HARTOG, $2001: 72$ )

Or, dans la géographie des Histoires, «cette symétrie, une fois posée, permet au narrateur de penser les relations de l'Égypte et de la Scythie selon les modalités du même et de l'inversion » (HARTOG, 2001 : 75). L'Égypte est alors perçue comme un reflet inversé de la Scythie.

D’une certaine manière, on retrouve dans le récit d'Ella Maillart une structuration de l'espace équivalente où, cependant, la ligne qui sert d' «équateur» n'est pas une ligne horizontale - comme celle qui, dans les Histoires, traverse la Méditerranée pour diviser le monde d'Hérodote en deux hémisphères symétriques - mais une ligne verticale, qui sépare l'Occident de l'Orient (et dont on pourra d'ailleurs se demander quel est exactement le point de passage). Et l'on peut ainsi penser l'espace du voyage «selon les modalités du même et de l'inversion », où l'Asie proposerait une sorte de reflet inversé de l'Europe. 
Ainsi, dans cette géographie où la limite entre l'Occident et l'Orient sépare deux mondes symétriques, il semble que la Suisse trouve dans l'Afghanistan son reflet oriental. En effet, l'Afghanistan, n'est-il pas lui-même «une Suisse asiatique » (p. 186) ? Le ciel audessus de Bamiyan, $d$ ' « un bleu riche et profond », n'est-il pas « semblable à celui qui domine les champs de neige en Suisse » (p. 264) ? Dès lors, on comprend que ce soit une «contrée » où, avant même son départ, Ella Maillart prévoit qu'elle se sentira «à l'aise » (p. 23). On comprend aussi qu'en franchissant la frontière afghane, les deux voyageuses, «pleines d'une joie triomphante » (p. 184) soient grisées par l'euphorie : non seulement elles approchent du but de leur voyage, mais elles ont aussi le sentiment d'avoir retrouvé leur patrie : «nous avions atteint "notre" pays » (p. 192) ${ }^{5}$.

Cependant, si l'Asie offre une image de l'Europe, et l'Afghanistan, de la Suisse, les reflets ne sont pas équivalents. Selon la logique du même et de l'inverse qui caractérise la structure symétrique de l'espace, l'Asie présente au contraire à l'Europe un reflet inversé. Ainsi, à l'Europe moderne, l'Asie propose l'image d'une Europe ancienne ; à l'Europe menacée, l'image d'une Europe en paix. Il suffit de comparer le « lac gelé » de l'Engadine (p. 21) à son double symétrique, le lac du Band-i-Amir en Afghanistan (p. 278) : si les deux descriptions offrent bien le même type de paysages lacustres au cœur des montagnes, néanmoins, au spectacle d' « austère désolation » que présente le premier, s'oppose le « décor inoubliable » de l'autre. Aussi, l'on peut comprendre qu'en découvrant ce dernier, Christina s'exclame : « J'aime cet endroit, [...] le visage souriant de surprise ».

Aussi, contrairement à ce que peut croire Ella Maillart au début du récit, le voyage en Orient relève-t-il moins d'un départ vers «l'étranger » (p. 23), que d'un retour vers un pays originel. Or, les premiers récits de voyages - les récits de pèlerinage médiévaux - concevaient eux aussi le voyage «non pas comme la recherche d'un ailleurs, mais comme un retour à la patrie perdue » (WOLFZETTEL, 1996 : 11). Pour les pèlerins, en effet, tout voyage menait même de façon symbolique - à cette patrie perdue du christianisme qu'était Jérusalem. Et, sur le chemin qui conduit Kini et Christina en Afghanistan, nombreux sont les lieux saints qui apparentent leur voyage à un pèlerinage, qu'il s'agisse du tombeau d'Oldjaytu (p. 131), de la mosquée de Mechhed (p. 167), ou encore du ziarat d'Hazrat Ali (p. 278). Cependant, il est un lieu qui, plus que tout autre - et plus encore, même, que Jérusalem - incarne la patrie perdue :

\footnotetext{
${ }^{5}$ Dans Croisières et caravanes, Ella Maillart remarque d'ailleurs que le « Pamir », la région qu'elle a toujours convoitée, « est le synonyme d'Alpes, pâturage » (MAILLART, 2001b : 198).
} 
c'est le Jardin d'Éden. Or, pour les deux voyageuses, il semble bien que le voyage en Asie doive moins les éloigner de l'Europe que les rapprocher d'un Âge d'or ; moins les conduire en Afghanistan, que leur permettre d'atteindre une Suisse édénique.

$*$

\subsection{L'Éden}

Comme Kini le rappelle à Christina au terme de La Voie cruelle, sa quête d'un refuge éloigné de l'Europe ne date pas de leur voyage, mais remonte aux lendemains de la Première Guerre Mondiale :

Quoique trop jeune pour avoir été directement affectée par la Première Guerre mondiale - comme Gerbault dont les trois meilleurs amis périrent à son côté -, je crois que j'ai indirectement souffert de ce que mes amis ont traversé. Cela m'a influencée. La dernière guerre m'expédia sur les mers, à jamais débarrassée de mes illusions sur notre civilisation. (p. 309)

En effet, même si Ella Maillart, «trop jeune » à l'époque, n’a pas conservé de souvenir direct des affrontements de 1914-1918, elle n'en a pas moins conçu une horreur de la guerre confortée, tout d'abord, par « la lecture consternante des livres de Barbusse ou Georges Duhamel sur l'imbécillité et la cruauté de la Première Guerre Mondiale » (BOUVIER, 1991 : 164), puis par son amitié avec le navigateur Alain Gerbault : «aviateur pendant la Grande Guerre, lui et trois de ses compagnons avaient décidé de gagner le Pacifique et d'abandonner pour toujours un continent où de telles guerres étaient possibles » (MAILLART, 2001b : 37). Cependant, ces trois amis «périrent à ses côtés » pendant le conflit, et Gerbault dut mener ses navigations en solitaire.

À l'exemple de ce dernier, c'est tout d'abord «sur les mers », et plus particulièrement vers le Pacifique, qu'Ella Maillart oriente elle aussi sa quête d'un paradis terrestre. Cependant, ses navigations, commencées sur le lac Léman et poursuivies en Méditerranée, ne la conduiront jamais aussi loin. Il lui faudra interrompre, en 1926, une traversée de l'Atlantique, avant d'abandonner ses aventures maritimes, un an plus tard, après la défection de Miette Saussure, sa camarade de navigation. Dès lors, ce n'est plus par mer, mais par voie de terre, qu'Ella Maillart entreprend de trouver l'Éden, ne le situant plus sur une île du Pacifique, mais dans les montagnes de l'Asie.

Il est intéressant de noter que, dans l'histoire personnelle d'Ella Maillart, la recherche de l'Éden, suit en sens inverse la localisation de ce même lieu dans l'histoire de l'humanité. 
En effet, avant la découverte du Nouveau Monde et l'exploration des îles du Pacifique, qui deviendraient ses points d'ancrage aux XVIe et XVIIIe siècles, le Paradis était généralement situé en Asie par les auteurs médiévaux.

O ponto de partida para as "visões" medievais do Paraíso encontra-se, naturalmente, no Gênese, 2, 9-25 e 3, 1-24, onde se narra como o Senhor Deus, tendo criado o homem, em quem insuflou o fôlego da vida e o fez assim alma vivente, plantou para sua habitação um horto "da banda do Oriente". (BUARQUE DE HOLANDA, 1996 : 150)

En cherchant l'Éden en Orient, les textes d'Ella Maillart reviennent donc au «point de départ des "visions" médiévales » du mythe. Ainsi, ce n'est pas seulement par l'itinéraire qu'elle se propose, mais aussi par la destination édénique qu'elle fixe à son voyage, qu'Ella Maillart s'affirme héritière des voyageurs du passé.

Bien sûr, il ne se trouve plus, au XXe siècle, de voyageur pour croire en l'existence physique du Paradis perdu ; néanmoins, Ella Maillart, devant la tombe du roi Kabus, estime qu'à une époque reculée «un âge d'or florissait alors dans cette partie du monde » (p. 154). De même, bien qu'elle avoue que cet objectif ait peu à peu faibli pour n'être plus qu'un «prétexte», Ella Maillart reconnaît poursuivre encore sa « recherche d'une édénique tribu montagnarde » (p. 59), qu'il s'agisse des montagnards du Kafiristan ou des «Lolos du Tibet oriental » (p. 23).

Mais c'est surtout par son caractère ascensionnel que le voyage d'Ella Maillart se distingue d'un simple déplacement géographique pour prendre la dimension d'une quête de l’Éden.

Au Moyen-Âge, la localisation du Paradis Terrestre en Orient valait à l'Asie de figurer en haut des mappemondes..

Do desejo explicável de atribuir-se, nas cartas geográficas, uma posição eminente ao Paraíso Terreal, representado de ordinário no Oriente, de acordo com o texto do Gênese, é bem significativo o modelo de mapa-múndi mais correntemente usado. Modelo este, em que, no hemisfério conhecido, Europa e África ocupam sempre a metade inferior, ao passo que a Ásia se situa acima dos demais continentes. (BUARQUE DE HOLANDA, $1996: 149$ )

Ainsi, sur les cartes géographiques du Moyen-Âge, l'Est occupe la place qu'on attribuera, plus tard, au Nord. Cette division du monde en deux hémisphères, dans laquelle l'Asie occupe la moitié supérieure, et l'Europe et l'Afrique se partagent la partie inférieure, 
confère au voyage qui mène de l'Occident à l'Orient le caractère d'une ascension ou, plus précisément - puisqu'en s'approchant du Paradis, le déplacement géographique vers l'Est se double d'un sens spirituel - le caractère d'une élévation.

Or, le voyage d'Ella Maillart vers l'Afghanistan semble bien, lui aussi, dès le départ, épouser un mouvement ascensionnel.

Silvaplana, dans l'Engadine, fut le tremplin d'où notre imagination s'était échappée vers le sud-est jusqu'aux plus grandioses versants de l'Asie. Mais notre réel envol eut lieu au col du Simplon d'où, zigzaguant, virant sur l'aile, plongeant en vrille au flanc de la montagne et traversant un sombre défilé, nous gagnâmes l'étranger. (p. 28)

Grâce au double sens du mot «aile »- et la Ford dispose en effet de larges ailes à chacune de ses roues - la voiture se voit attribuer ici l'envergure d'un avion. Et la plongée « en vrille au flanc de la montagne » évoque moins la descente d'une route en lacets, que le vol tournoyant d'un planeur. Cependant, le mouvement de descente se trouve amorti, comme si les deux voyageuses se servaient de ces premiers cols comme d'un « tremplin », comme si, au lieu de tomber, elles prenaient leur «envol». Et c'est d'ailleurs ce même mouvement ascensionnel qui, après la Maloja et le Simplon, accompagne le franchissement d'au moins trois autres cols. «Par trois fois, d'ailleurs, nous allions monter du niveau de la mer vers de hauts cols : de la mer Noire en Arménie, de la Caspienne au Khorasan et du Turkestan en Afghanistan » (p. 79).

Chaque franchissement de ces « hauts cols » marque l'accès à un plateau plus élevé ; et, quand Ella Maillart atteint le premier des trois, elle prend alors brusquement du recul, donnant à voir l'espace où va s'inscrire son voyage comme sur une immense carte des reliefs.

Nous avions atteint le plateau anatolien à l'intérieur des parenthèses montagneuses formées par le Taurus et la chaîne Pontique. Une ceinture similaire encercle le plateau iranien au nord et au sud. Et un troisième cerceau colossal, formé par les chaînes du Kunlun et les Himalayas, contient le haut pays du Tibet. [...] Anatolien, iranien, tibétain : trois vastes plateaux en enfilade, chacun d'eux plus grand et plus élevé que le précédent, chaque enchevêtrement de montagnes abritant des groupes d'hommes très différenciés. (p. 87-88)

Les deux voyageuses n'atteindront pas le plateau tibétain; il reste, néanmoins, que cette ascension par paliers double le voyage horizontal d'un mouvement vertical, comme si les deux voyageuses ne laissaient pas seulement l'Europe derrière elles, mais aussi en bas, gagnant par étapes un Orient situé de plus en plus haut. 
Le mouvement ascensionnel, qui caractérise la progression des deux voyageuses vers l'Orient, s'accompagne par ailleurs d'un progressif allègement; il suffit, pour s'en rendre compte, d'opposer la pesanteur de l'Engadine, au début - dont le « ciel bas et sombre pesait sur la vallée », et dont le lac était « recouvert de nombreuses couches de neiges » (p. 21) - à la légèreté du spectacle que, à l'autre extrémité du voyage, les deux voyageuses découvrent en franchissant la frontière afghane.

Dans le noir de la nuit, nos phares formaient un trou de lumière. Surgissant de l'obscurité, d'immenses oiseaux à poitrail blanc nageaient sans effort sur notre fleuve lumineux. Ils se maintenaient ou se rétablissaient parfois à l'aide d'un bout d'aile brune ; à part cela on ne voyait que la pâleur de leur vaste envergure. Pouvions-nous souhaiter plus somptueuse escorte ? Peutêtre que Simurgh le noble vautour se trouvait parmi eux ? (p. 184)

Il semble qu'au premier envol de la voiture, au col du Simplon, réponde celui de ces «immenses oiseaux à poitrail blanc», comme si les deux voyageuses s'étaient finalement jointes à leur cortège. À quelle espèce appartiennent ces volatiles ? «Dans le noir de la nuit », on ne distingue qu' « un bout d'aile brune », on n'entrevoit que «la pâleur de leur vaste envergure », comme s'ils étaient moins des animaux de chair et d'os qu'une sorte de vol pur ; ils semblent d'ailleurs voler «sans effort», comme s'ils étaient sans poids. On dirait bien que les deux voyageuses ont quitté la pesanteur pour finalement atteindre l'essence même de la légèreté.

Mais comment ne pas évoquer aussi, parmi ces mouvements d'ailes indistincts, la figure d'êtres mythiques? Ella Maillart pense à «Simurgh le noble vautour», dont le souvenir lui était revenu une première fois près du mont Demavend (p. 144); mais, au bout de leur progressive ascension, comment ne pas penser que les deux voyageuses sont accueillies par une «somptueuse escorte » d'anges aux portes de l'Éden ? En effet, la Genèse précise qu'après en avoir chassé Adam et Eve, Dieu « mit des Chérubins devant le jardin de délices, qui faisaient étinceler une épée de feu pour garder le chemin qui conduisait à l'arbre de vie » ${ }^{6}$ (Genèse, III, 24). Et si les battements d'ailes, dans La Voie cruelle, renvoient aux bibliques Chérubins, le «fleuve lumineux » que produisent les phares de la Ford au milieu de la nuit, évoque quant à lui l'« épée de feu » protégeant l'arbre de vie.

\footnotetext{
${ }^{6}$ Toutes les citations de la Bible sont extraites de la traduction de Lemaître de Sacy, éditée par Philippe Sellier à Paris, en 1990, au éditions Robert Laffont, dans la collection « Bouquins ».
} 
Les anges, d'ailleurs, même s'ils ne sont pas nommément désignés ici, ne sont pas absents du récit. En passant près d'un distributeur d'essence, en Turquie, Ella Maillart se souvient que, lors d'un précédent voyage, tombée en panne au même endroit, sans essence ni argent, elle s'était demandé : «Quel ange va venir à notre secours ? (p. 38) ; aussitôt, une voiture s'était arrêtée, dont le propriétaire avait accepté d'avancer le prix de l'essence. «Lorsqu'il nous donna sa carte de visite, son prénom était Angelo ». Et Christina elle-même n'est-elle pas couramment appelée «l'ange déchu » par un de ses amis (p. 25) ? Or c'est bien pour aider cet ange à reprendre son vol, qu'Ella Maillart accepte d'emmener Christina avec elle en Orient.

Toujours est-il que, de l'Europe à l'Afghanistan, il semble bien que les deux voyageuses s'éloignent progressivement du pays de la Chute, pour finalement atteindre, au bout d'une lente ascension, parmi des bruissements d'ailes, les portes du Paradis.

Ainsi, La Voie cruelle s'inscrit bien dans une tradition : non seulement du fait que tout récit de voyage en Asie se place au cœur d'une intertextualité riche et complexe, mais aussi parce qu'Ella Maillart fixe à son voyage un objectif qui était celui des premiers voyageurs : la quête d'une patrie perdue. En effet, dans sa représentation symétrique de l'Occident et de l'Orient, Ella Maillart fuit moins la Suisse qu'elle ne cherche, en Afghanistan, une Suisse originelle. Les deux voyageuses non seulement quittent l'Europe menacée pour trouver refuge en Asie, mais surtout, elles remontent progressivement, par plateaux, du monde de la Chute vers l'Éden.

Cependant, au fil de la route, il semble bien qu'Ella Maillart soit contrainte de revoir sa vision de l'espace : et que, si l'Orient offre toujours à l'Occident un reflet symétrique plus ancien et plus pur, cela ne doive durer que peu de temps encore. 


\section{Le fil de la route}

La quête de cette patrie perdue qu'est l'Éden, et la structure symétrique de l'espace, montrent assez combien le voyage d'Ella Maillart est moins une fuite qu'un retour, et qu'en allant vers l'Orient, c'est en fait vers un autre Occident qu'elle dirige ses pas. Elle cherche moins à quitter l'Europe, qu'à retrouver une Europe originelle. Or, ce qu'elle attend de l'Asie, c'est qu'elle lui permette de mieux comprendre les maux dont souffre l'Europe : dans l'Asie en paix, elle espère mieux comprendre ce qui a pu conduire l'Europe au bord de la guerre. Aussi, l'observation du bord des routes, si caractéristique du récit de voyage, se double-t-elle dans La Voie cruelle d'une sorte de relevé des symptômes : il s'agit d'établir le diagnostique d'un corps malade - l'Europe - en le comparant à l'état d'un corps plus sain - l'Asie. Au long du chemin, Ella Maillart va ainsi remonter de la guerre à l'industrialisation, et de cette dernière à ce qu'elle nomme les «besoins artificiels », mesurant mieux alors toute l'étendue du mal et les difficultés de lui porter remède.

\subsection{Les bas-côtés}

Il est intéressant de relever qu'à Trébizonde, véritable point de départ de son voyage vers l'Asie, Ella Maillart croise le souvenir de Marco Polo « de retour de Chine », l'imaginant « aussi joyeux que les misérables Dix Mille lorsqu'il vit enfin les eaux de sa Méditerranée » (p. 80). Ainsi, au moment où elle-même prend la route de l'Orient, Ella Maillart évoque le retour de Marco Polo vers sa Venise natale, comme si elle voyageait à contre-courant de l'illustre vénitien. Comme si, au moment de quitter l'Europe, elle éprouvait le sourd désir de faire demi-tour. Cette impression de voyager à contre-courant est d'ailleurs redoublée par d'autres souvenirs : non plus ceux de Marco Polo, mais ceux d'Ella Maillart elle-même. La voyageuse, en effet, quelques années auparavant, a déjà suivi cette route dans l'autre sens : « Il y a deux ans, j'ai fait le voyage des Indes en Turquie à bord de camions et d'autobus » (p. 22). Et si Marco Polo dut être «joyeux » de retrouver «les eaux de sa Méditerranée », Ella Maillart éprouve de la nostalgie à quitter cette mer à laquelle elle accole, elle aussi, un article possessif.

Ses rives devenant pourpres à la fin du jour, je reconnus ma Méditerranée rieuse, la mer enjôleuse avec des arbres penchés sur sa grève ; cette « mer du Milieu » dont l'atmosphère et la culture sont à la mesure de l'homme. 
Comment pouvais-je être assez égarée pour tourner le dos au monde auquel j'appartenais ? (p. 65)

Que ce soit dans la figure du grand voyageur rentrant à Venise, ou dans cet adieu crépusculaire qui semble remettre en question son propre départ, Ella Maillart insiste sur l'attachement qu'éprouve le voyageur pour sa terre d'origine, sa « mer du Milieu », son centre - son port d'attache - autant que sur l'attirance que les pays lointains peuvent exercer sur lui. Sur les forces centripètes de l'appartenance, autant que sur les forces centrifuges de l'évasion. «Bien que notre regard intérieur fût fixé sur un but impatiemment désiré, nous partions lentement, et la nostalgie de l'immense désolation du désert persan n'empêchait pas nos yeux d'être sensibles au Pays romand» (p. 35). Comme si, d'emblée, pour Kini et Christina, ce voyage relevait moins d'un départ volontaire que d'un arrachement.

Aussi le voyage ne s'inscrit pas entre un pôle répulsif et un pôle attractif comme pourrait le faire penser, au début du récit, l'évocation d'une Europe en guerre et d'une Asie en paix, d'un Occident décadent et d'un Orient édénique. En effet, Ella Maillart continue malgré tout d'être attachée à l'Europe qu'elle quitte ; et si elle fuit une chose, c'est moins l'Europe que la guerre. Car non seulement, à l'heure du départ, elle mesure tout ce qui l'attache au continent européen, mais aussi, combien le sort de l'Europe la touche.

En effet, l'état de l'Europe à la veille de la guerre inspire à Ella Maillart une profonde tristesse. Non seulement l'évocation par Christina, au début du livre, de « ses amis tchèques » conduit Ella Maillart «en pensée à Prague » (p. 21), mais encore les séjours qu'elle fait, avant son départ, à Paris, à Londres et à Munich, lui permettent de prendre toute la mesure du «drame européen » (p. 29). À Paris, tandis qu'elle chemine sur les Champs-Élysées, le ciel au-dessus d'elle est «bleu clair, chaud et gai »; cependant, alors qu'elle s'engage dans la rue Montaigne, une sorte de violent orage intérieur éclate, dont les eaux n'inondent pas la ville, mais les joues d'Ella Maillart.

Mais quoique consciente de la splendeur du moment, tout à coup ma gorge
se contracta. Des larmes inondèrent mes joues. Bouleversée, à moitié
aveuglée, je cherchai un banc où me remettre. Aussi violente
qu'impersonnelle, cette émotion se transforma lentement en une pensée : en
moi quelque chose souffrait d'une manière aiguë pour Paris... comme si la
chair et l'esprit de Paris étaient écrasés, martyrisés, écartelés, et comme si
ma compassion était devenue assez grande pour recouvrir l'ensemble de
toute cette ville aimée et soudain torturée. (p. 29)

Pour évoquer le calvaire de Paris, Ella Maillart recourt au vocabulaire du martyr : « la chair et l'esprit de Paris étaient écrasés, martyrisés, écartelés », la capitale, «torturée ». 
L'«émotion » qui l'étreint alors ne provient pas d'un drame intime : au contraire, elle est «aussi violente qu'impersonnelle»; elle n'est pas confinée aux limites d'une histoire individuelle, mais «assez grande pour recouvrir l'ensemble de toute cette ville ». L'émotion qu'elle éprouve est en fait une «compassion»: Ella Maillart com-patit, elle souffre avec Paris. Dans les larmes d'Ella Maillart, c'est en fait tout Paris qui, à cet instant-là, exprime sa souffrance. Aussi, devant l'Europe menacée, Ella Maillart n'éprouve pas de rejet comme si elle ne se reconnaissait plus dans ce continent : au contraire, tout ce passage montre combien elle s'identifie encore à lui. Il semble bien qu'à cet instant, Ella Maillart prenne sur elle toute la souffrance de l'Europe, et qu'elle s'attribue pour mission d'obtenir la rédemption de tous les siens.

Des sentiments d'appartenance et de compassion dotent ainsi, dès le départ, le voyage en Orient d'une dimension moins individuelle que collective. Et si la Première guerre mondiale, comme nous l'avons vu, avait poussé Ella Maillart à prendre le large, en suivant l'exemple d'Alain Gerbault, pour fuir sa «civilisation », la Seconde l'expédie sur les routes pour une autre raison. En effet, quand cette dernière éclate, Ella Maillart est bien consciente de ne pas être partie cette fois-ci dans le même état d'esprit.

La dernière guerre m'expédia sur les mers, à jamais débarrassée de mes illusions sur notre civilisation. Cette guerre-ci me force à chercher quelle est la signification de ce monde, quel est le commun dénominateur de chacun de nous, la base sur laquelle on peut recommencer à vivre. (p. 309)

Ella Maillart n'a pas quitté l'Europe par rejet d'un continent qui lui inspirerait du dégoût, mais bien pour trouver un solution, non seulement personnelle mais commune, au malaise dont souffre ce même continent, une «base sur laquelle on peut recommencer à vivre ».

Ce qu'Ella Maillart recherche dans son voyage, c'est moins un refuge qu'un remède, c'est moins l'oubli qu'un poste d'observation. Elle n'abandonne pas l'Europe pour l'Asie : en Orient, elle veut comprendre les maux dont souffre l'Occident et leur trouver une solution.

Mais, demandai-je à Christina, que penserait Nicole si je lui disais que j'étudiais l'Afghanistan (contrée jusqu'ici très peu touchée par l'Occident) parce que je voulais avant tout observer l'Europe sous un nouvel angle, afin de comprendre la cause profonde de notre instabilité ? Et qu'ayant ainsi ausculté notre continent, j'espérais apprendre comment mes contemporains avaient cessé de vivre en accord avec leur cœur? (p. 179) 
Si Ella Maillart veut étudier l'Afghanistan, c'est pour observer l'Europe « sous un nouvel angle ». Réduire par l'étude la distance qui sépare d'une culture étrangère afin de prendre, à l'égard de sa propre culture, une distance suffisante pour en faire un objet d'étude : ce que décrit ici Ella Maillart, c'est le rapprochement et la distanciation qui composent les deux grands moments de l'expérience ethnographique ${ }^{7}$ - se rapprocher des autres, pour se distancier des siens et mieux les observer.

On comprend mieux, alors, l'impression précédemment relevée d'un voyage à contrecourant: si Ella Maillart se rend en Asie, elle n'en quitte pas pour autant l'Europe des yeux ; si, à travers le pare-brise, c'est vers l'Orient qu'elle dirige ses regards, néanmoins, dans le rétroviseur, elle ne perd jamais de vue l'Europe qu'elle a laissée derrière elle. Le voyage est en fait doublement orientée : s'il prend la direction de l'Est, il continue néanmoins de regarder vers l'Ouest.

Dans le chapitre précédent, nous avons vu qu'Ella Maillart mesure les progrès du voyage en relevant, sur les bords de routes, les stigmates du fascisme et les prémices de l'Orient. Cependant, quand les deux voyageuses engagent leur voiture sur les routes d'Asie, Ella Maillart se montre sensible à un troisième ensemble de signes : ceux qui révèlent une progressive industrialisation de l'Orient. Or, c'est dans cet espace de transition entre l'Occident et l'Orient que représente l'Iran et, en Afghanistan, dans la vallée du Poul-iKhumri, qu'Ella Maillart parvient à comprendre le mieux le problème de l'Occident : en y observant le même mal à l'œuvre sur des corps plus sains.

Sur le bord de la route, Ella Maillart relève à certains détails la pénétration de produits industriels au milieu de techniques plus archaïques.

L'« ambiance âgée » de la région était encore soulignée par le beau pont en dos d'âne de Koprikoi, construit il y a quelques sept cents ans par les Seldjoukides. Tout près, cinq paires de bœufs tiraient une charrue comme autrefois ; mais l'outil portait une marque de fabrique rouge et soviétique : Krassni Zavod. (p. 93)

Voyager vers l'Orient, c'est remonter le temps : le retard des pays asiatiques en matière technique, le recours à des méthodes de travail préindustrielles, donnent en effet au

\footnotetext{
${ }^{7}$ Dans Nous et les autres, Todorov divise à leur tour chacun de ces moments en deux étapes, décomposant ainsi l'expérience ethnographique de Lévi-Strauss en quatre temps (TODOROV, 1984 : 124-125).
} 
voyageur européen la sensation de faire un bond de «sept cents ans » en arrière, de revenir à l'époque des «Seldjoukides ». Mais, même si la traction de la charrue est encore archaïque, cet « outil » introduit quant à lui une note de modernité, dont le « rouge » criard - autant que la conjonction «mais »- dit assez combien il détone dans «l'ambiance âgée » du paysage.

Or, cette marque du progrès n'introduit pas seulement dans un monde archaïque un élément étranger; elle est aussi un élément de rupture avec le passé. C'est ainsi qu'Ella Maillart perçoit d'autres éléments industriels, comme ces vestiges de la Première Guerre mondiale abandonnés sur la route de Bayezid :

Comme des jouets abandonnés dans l'herbe rude de la steppe, des rails tordus et de petites locomotives renversées jonchaient la plaine, vestiges de la ligne bâtie par les Russes pendant la guerre de 1914-1918. Dans cette contrée dépourvue d'industries modernes, il était curieux de voir des rails servir de linteaux de porte, de poutres ou de solives, et même de barrières autour des monuments aux morts de la guerre - car il n'y a pour ainsi dire pas d'arbres en Arménie. Çà et là, le long de la piste, des plaques de chaudière remplaçaient le ciment écroulé d'un petit pont de canal. (p. 95)

Plusieurs éléments retiennent notre attention dans ce passage. D’une part, le caractère apparemment inoffensif de ces «petites locomotives » abandonnées comme des «jouets », semble réduire à des proportions dérisoires les machines des hommes, comme s'ils n'étaient jamais que de grands enfants s'amusant avec des trains électriques. D'autre part, ces « vestiges» du chemin de fer, répandus dans «l'herbe rude de la steppe », dénoncent la pollution par les hommes du milieu naturel ; cependant, dans le contexte du récit de voyage, la «steppe » est aussi un espace mythique : elle évoque les tribus nomades de l'Orient - les Mongols descendants de Gengis Khan - dont le mode de vie était au moins aussi « rude » que l'herbe qu'elles foulaient. Ainsi, si les débris répandus dans la steppe portent atteinte à la nature, ils annoncent aussi la fin d'un mode de vie ancestral où l'homme s'inscrivait harmonieusement dans son environnement. De plus, les déchets ont non seulement souillé la nature, ils ont aussi contaminé les constructions humaines, incorporant aux maisons, aux monuments, aux ponts, des matériaux industriels - «rails », «plaques de chaudières »-qui leur sont étrangers, et dont la présence frappe d'autant plus dans des régions qui, comme l'Arménie, en sont normalement dépourvues. Aussi, les produits industriels introduisent-ils non seulement un corps étranger dans un décor archaïque : ils en contaminent aussi petit à petit toutes les constructions.

Plus loin, Ella Maillart relève d'ailleurs comment toutes sortes de débris importés par le rail se sont non seulement mêlés, comme dans la steppe arménienne, aux matériaux 
traditionnels de construction, mais les ont entièrement remplacés. "Tel un malsain champignon importé par le rail - symbole du progrès dit-on ! - un hameau, bâti avec le métal de bidons d'essence aplatis, rouillait au bord de la route, exacte réplique de ce qu'on peut voir dans la zone de Paris»(p. 146). Ainsi, Ella Maillart montre combien le développement industriel, diffusé par le développement conjoint du chemin de fer, finit par atténuer les différences entre pays : le même «hameau » sordide, «bâti avec le métal de bidons d'essence aplatis », ne permettant plus de distinguer par ses matériaux de construction un bidonville iranien d'un quartier de «la zone de Paris ». À terme, l'Orient et l'Occident finiront par présenter le même visage : non plus, comme par le passé, parce qu'il existe une filiation entre les deux continents, mais parce que les progrès de l'industrialisation les auront recouverts des mêmes déchets. Ella Maillart annonce en quelque sorte le constat pessimiste de Tristes Tropiques : «Ce que d'abord vous nous montrez, voyages, c'est notre ordure lancée au visage de l'humanité » (LÉVI-STRAUSS, 1955 : 36). Aussi, tout autant - et peut-être même plus que la guerre, c'est cette évolution dont le voyage lui fait découvrir les causes et les effets qu'Ella Maillart semble ici condamner.

\subsection{Routes}

Le chapitre 11, « Routes », est un des seuls qui ne portent pas le nom d'un lieu géographique. Comme les chapitres 1, 2 et 19, respectivement intitulés : «L'idée », «Le départ » et «La frontière », il reprend une composante essentielle du voyage et permet, sous la variété des noms géographiques, d'en jalonner les principales étapes, d'en suivre la structure élémentaire. Cependant, l'usage du pluriel suggère ici qu'il existe une pluralité de routes, non seulement par leurs nombres, mais aussi par leurs natures : des routes non seulement nombreuses mais encore variées.

Cela est particulièrement évident quand, en Iran, les deux voyageuses empruntent les routes qui conduisent à Bayezid-la-Vieille et à Bayezid-la-Neuve. D'une part, «huit kilomètres de piste souvent réduite au roc poli par des siècles d'usage » aboutissent à «la fière forteresse du vieux Bayezid » (p. 96); d'autre part, « la route vierge pas encore ouverte à la circulation » conduit à « Bayezid-la-Neuve» dont « les cubes gris de ses quelques maisons se groupaient autour de bœufs et de camions » (p. 96). Il y a donc au moins deux variétés de 
routes, qui se distinguent autant par leur état d'usure que par les lieux auxquels elles conduisent : les routes anciennes, qui aboutissent aux murs de pierres des forteresses ; et les routes en construction, qui mènent aux «briques de glaises récemment démoulées » des villes de passage. Il est des routes par lesquelles on pénètre dans le passé de l'Orient ; et d'autres par lesquelles il semble que ce soit au contraire le présent qui pénètre dans l'Orient. Il y a donc une ambiguïté de la route qui, tout à la fois, relève de la tradition et de la modernité.

Si les deux variétés de routes ne conduisent pas aux mêmes lieux, c'est aussi qu'elles ne suivent pas les mêmes tracés. «Les ingénieurs de la route» (p. 96) ont en effet pour objectif de réduire les distances; ainsi, «la route de Chalus » à laquelle œuvrent des « ingénieurs suisses » correspond au «trait d'union le plus rapide entre Téhéran et les riches provinces au sud de la Caspienne » (p. 120), et la route à laquelle travaille l'ingénieur Adnan Bey à Bayezid représente pour les Iraniens « le plus court chemin pour aller en Europe » (p. 96). Les nouvelles routes permettent non seulement de relier deux régions éloignées, voire même deux continents, mais elles permettent aussi de les rapprocher: elles permettent non seulement de réduire les distances, mais aussi, comme nous le verront, de réduire les différences.

Enfin, c'est aussi leur fréquentation qui distinguent les routes anciennes des routes modernes, ou plutôt les «routes des grandes caravanes» des «lignes actuelles de communication »: «Les routes des grandes caravanes d'Asie centrale sont mortes et l'étranger qui visite Herat se sent bien loin des lignes actuelles de la communication » (p. 203). En effet, les routes peuvent être, d'une part, des routes «mortes », c'est-à-dire désertes, peu fréquentées, ou bien des voies de «communication», de grands axes de circulation. Les unes se prêtent à des moyens de transports traditionnels - les bêtes de somme des « caravanes »- les autres, à des moyens de transport modernes qui, dans le livre, occupent une place importante : la «Ford » qui emporte des deux voyageuses, et les «lorries » destinés au transport de marchandises. Or, ces deux moyens contribuent autant que les nouveaux tracés à rapprocher les continents.

C'est le premier voyage qu'Ella Maillart effectue en voiture. Plus habituée au rythme des caravanes, il lui faut d'ailleurs du temps pour se défaire de ses anciens réflexes. «Oubliant que j'étais en voiture, à plusieurs reprises mes réactions furent celles du lent voyageur de caravanes » (p. 84). Et les comparaisons qu'elle établit entre la Ford et les deux moyens de transports qu'elle connaît le mieux - les navires et les bêtes de somme - éclairent d'ailleurs les changements qu'introduit l'automobile dans sa manière de voyager. 
Pour Ella Maillart, la Ford est tout d'abord comparable à un bateau : «étroite au radiateur et large en poupe, c'était une embarcation qui, tout au long de nombreux mois, allait nous ouvrir l'Europe et l'Asie dans une fuyante vague d'étrave » (p. 35). La voiture s'assimile d'autant plus au navire que, contrairement aux transports collectifs qui, camions ou autobus, imposent au voyageur de suivre un itinéraire fixe et, lors des arrêts, « de rester à proximité du camion au lieu de vagabonder à sa guise » (p. 22), la Ford le laisse libre de s'arrêter quand il le décide et d'aller où bon lui semble.

\begin{abstract}
N'est-il pas enthousiasmant que de faire face au printemps et à la grandroute lorsqu'on est à même de rouler pendant des milliers de kilomètres, à même de camper ou de manger, de s'arrêter ou de changer librement d'itinéraire ? Il n'y a que lorsqu'on quitte un port à son propre bord qu'on est encore plus ému ; car en mer, l'immensité s'offre sans restriction, aucune route tracée ne s'impose à la quille. (p. 42)
\end{abstract}

Ainsi, tant par sa forme que par le sentiment de liberté qu'elle procure, la Ford ravive dans la mémoire d'Ella Maillart ses souvenirs de vagabonde des mers, et donne au départ en voiture des allures d'appareillage.

Cependant, la voiture est aussi comparable aux bêtes de somme, lorsque Christina fixe «au radiateur quelques grosses perles bleues comme on en voit au cou des animaux qu'on veut protéger du mauvais œil » (p. 159). Mais la comparaison ne saurait aller au-delà, car la différence de vitesse est trop grande entre la voiture et les caravanes :

Il ne fallut que douze heures à notre huit-cylindres pour couvrir ces trois cent cinquante kilomètres de route de montagne, et non pas dix ou quinze jours selon l'état des bêtes de somme. Mais je me souvenais encore de deux voyages faits en caravane plus à l'Est, le long de cette même Route de la Soie, piste démesurément longue [...]. (p. 84)

Son expérience des caravanes permet à Ella Maillart de comparer le temps que mettent une «huit-cylindres» et des «bêtes de somme» à parcourir une même distance. Elle découvre alors que les distances dépendent avant tout du temps qu'on met à les parcourir : un voyage se chiffre moins en «kilomètres» qu'en «jours»; et une simple «route de montagne », selon le moyen de transport, peut aussi devenir une «piste démesurément longue $\gg$.

Cependant, Ella Maillart découvre aussi que cette vitesse présente un inconvénient : si l'on n'y prend garde, elle risque de priver le voyageur de cette liberté que la voiture lui avait tout d'abord accordée. En effet, « la plupart des automobilistes conduisent comme si c'était 
un crime de rompre la transe engendrée par le continuel élan des $80 \mathrm{~km} / \mathrm{h}$. Ils atteignent un état dans lequel ils perdent toute initiative, hormis celle d'aller de l'avant » (p. 49). Grisé par la vitesse («80 km/h »!), plongé dans une sorte de «transe », le voyageur abandonne « toute initiative » à son propre véhicule, comme s'il était dans un train ou dans un autobus, perdant ainsi cette liberté dont Ella Maillart faisait justement un des avantages de la voiture sur les transports en commun.

Mais la vitesse fait courir un risque encore plus grand. Ella Maillart en veut pour exemple le voyage effectué sur la même route, quelques années auparavant, par la baronne Blixen-Finecke ${ }^{8}$. Cette dernière «avait fait le trajet en un temps record. Mais elle avait peu vu, ne s'était arrêtée nulle part, et ses photos ne montraient que sa Ford dans le sable, dans l'eau, dans les foules, dans le désert » (p. 50). Aussi la voiture - et surtout la vitesse - risquet-elle de priver le voyageur de son principal attribut : voir; et de réduire l'immensité de l'espace à un périmètre réduit autour du véhicule. Les deux voyageuses ont bien conscience que leur voiture, instrument d'exploration, peut aussi devenir un facteur d'isolement.

Nous ne voulions pas davantage permettre à la vitesse de construire un «mur » isolant entre nous et la vie des alentours - bruits de voix, odeur de nouvelles épices sortant d'une ferme, fraîcheur d'une timide brise près d'une source. Ce voyage serait le nôtre et non celui de la voiture. (p. 49)

En même temps que la voiture, comme les nouvelles routes, raccourcit les trajets et rapproche les continents, elle risque fort de dépouiller les voyages de tout ce qui faisait leur saveur - les bruits, les odeurs - pour les réduire à de simples déplacements dans l'espace. Or, voyager, ce n'est pas seulement se déplacer : c'est avant tout voir, sentir et rencontrer. Et le rythme des caravanes, qui laissait le temps d'apprécier les paysages et, surtout, de faire des rencontres, s'accordait sans doute mieux au voyage que la vitesse des automobiles. En voyageant pour la première fois en voiture, Ella Maillart comprend que voyager, c'est aller lentement. Aussi se remémore-t-elle avec nostalgie ses anciennes pérégrinations et commence-t-elle, comme le feront d'autres voyageurs - en particulier Nicolas Bouvier - à faire l'éloge de la lenteur.

Avec le camion, comme avec la voiture, c'est tout un mode de vie nomade qui évolue :

\footnotetext{
${ }^{8}$ L'écrivain suédois Karen Blixen, auteur de La ferme africaine.
} 
Les chameaux disparaissent, remplacés par des camionnettes ferraillantes et grondantes qui se nourrissent d'un liquide huileux venu de Bakou ou de Birmanie. Les chefs de caravane ne se réunissent plus la nuit autour du feu du caravansérail pour écouter les histoires d'un conteur. Le sérail s'est mué en un garage où des chauffeurs habiles passent la nuit à faire des réparations sous leurs machines. (p. 254)

L'apparition du camion n'implique pas de changement que dans le mode de transport : non seulement les «camionnettes» remplacent les «chameaux », mais le «garage » s'est substitué au «caravansérail » et les «chauffeurs » aux «chefs de caravane ». On aurait pu croire qu'Ella Maillart, accoutumée aux caravanes d'Asie, regretterait cette évolution; cependant, il semble bien que chaque nouvel élément se soit substitué à l'ancien sans pour autant rien changer à l'ensemble, comme si l'on avait rénové les pièces d'une structure sans pour autant en modifier l'organisation. Et les «camionnettes » remplacent d'ailleurs si bien les «chameaux » qu'Ella Maillart les assimile, dans ce passage, à des animaux qui «se nourrissent d'un liquide huileux », préparant une métaphore qui transformera plus loin les camions en «"bêtes de somme" modernes» (p. 255). Aussi, Ella Maillart peut-elle se demander «quel poète chantera les camions d'Asie ? », dans une «moderne épopée » (p. 255) où le camion prendrait la place du chameau, sans plus changer la dimension héroïque du genre qu'il ne semble avoir modifié le mode de vie nomade.

Cependant, le regard qu'Ella Maillart porte sur ces mêmes camions évolue, quand ils ne sont plus perçus comme de simples «bêtes de sommes », mais comme des «lorries » (p. 115), destinés non seulement au transport de produits manufacturés, mais encore à celui d'usines entières :

«Selon moi, c'est la multitude des rudes conducteurs de camion sillonnant chaque nuit les routes d'Angleterre qui rendent cet âge mécanique », écrivit T. E. Lawrence à Robert Graves. En Iran, c'est également vrai. Les douzaines de filatures, de minoteries, de fabriques de cigarettes, de savon, de ciment, d'usines d'égrenage de coton, les raffineries de sucre, la station de radio ont toutes dû être importées, pièce par pièce, tout au long des interminables routes de ce pays. (p. 117)

Ainsi les camions sont non seulement les diffuseurs des produits industriels, mais encore les agents d'une progressive industrialisation de l'Orient, faisant entrer l'Asie dans cet «âge mécanique » dont parle T. E. Lawrence. 
De plus, si les camions répandent en Orient les usines de l'Occident, ils en diffusent aussi les idées. Une scène qu'Ella Maillart relève au bord d'une route iranienne est en cela très éclairante.

Nous arrivâmes près d'un camion renversé. Sur la route gisaient deux ou trois caisses colossales marquées ELEVATOR en russe et en anglais. L'ombre du châssis abritait deux hommes - seule tache sombre au cœur de l'immensité éblouissante. Il y avait plus de vingt-quatre heures qu'ils étaient là, attendant une équipe de secours. Le chauffeur iranien gémissait :

- C'est pas ma faute ! Qu'est-ce que je pouvais faire : Dieu l'a voulu !...

Le mécanicien russe, qui prenait une papiross dans la boîte de Camel offerte par Christina, répliqua :

- Dieu ? Pauvre bêta, de quoi parles-tu ? Tu sais aussi bien que moi que Dieu n'existe nulle part que dans ta tête !

Ainsi les idées, elles aussi, s'exportent de la Russie soviétique. (p. 117-118)

Ce passage présente un certain nombre de points communs avec celui dans lequel, plus haut, Ella Maillart évoquait les vestiges du chemin de fer éparpillés parmi les herbes de la steppe. Ici, les rails sont remplacés par «la route» et les locomotives par un «camion renversé »; mais il s'agit toujours d'évoquer le développement des transports et de montrer, par un accident, les épaves que l'ère industrielle sème sur son chemin. La souillure dont le camion renversé marque la blancheur du milieu naturel est ainsi révélée par «la tache sombre » dont «l'ombre du châssis » souille «l'immensité éblouissante ». D'autres éléments cependant s'ajoutent au tableau, qui sont d'ordre moins matériels que culturels: d'une part, les «caisses colossales marquées ELEVATOR en russe et en anglais » témoignent tout à la fois de la diffusion des techniques occidentales et des langues européennes dans le monde arabe ; d'autre part, le dialogue entre « le chauffeur iranien » et « le mécanicien russe » révèle la circulation des « idées », en particulier sur la question religieuse et l'existence de Dieu.

Et il semble bien que le Dieu des Arabes ne puisse longtemps résister aux nouvelles idées venues de l'Occident. C'est du moins ce qu'Ella Maillart voit, à Tabriz, dans le voisinage d'une mosquée et d'une salle de cinéma :

À nos pieds, à l'extrémité de la grande cour ouverte, là où le mihrab arqué indiquait la direction sacrée de La Mecque, trônait la cabine ininflammable d'un projecteur de cinéma, nouveau dieu dispensateur d'oubli à notre civilisation de masses. (p 110)

«Le mirhab arqué » et la «cabine » de projection semblent indiquer des directions opposées ; l'un montre depuis des millénaires «la direction sacrée de La Mecque », tandis 
que l'autre dispense l'« oubli»des anciennes valeurs ; mais ce duel entre l'ancien et «le nouveau dieu » - entre la religion et le cinéma - semble d'ores et déjà remporté par ce dernier, dont le symbole «trônait » au milieu de la cour, comme s'il y avait déjà établi son règne - d'autant plus résolument, d'ailleurs, qu'Ella Maillart insiste sur son caractère indestructible, « ininflammable ».

Ainsi, avec le développement des transports, les routes deviennent pleinement des voies de «communication », sur lesquelles circulent non seulement des produits, mais aussi des idées. Or, qu'il s'agisse de l'uniformisation des paysages, favorisé par l'usage des mêmes matières industrielles, ou de celle des modes de pensées, Ella Maillart montre comment le progrès technique conduit à l'effacement progressif des cultures orientales, au profit d'une seule et même « civilisation de masses » venue d'Occident.

Aussi, Ella Maillart est-elle consciente du double aspect de la route : à la fois ce qui permet - pour combien de temps encore ? - d'accéder aux beautés de l'Asie, mais aussi ce qui, au rythme des nouveaux moyens de transport, propage la civilisation de l'Occident vers l'Orient. Et si la route est encore ce qui permet de découvrir l'Ailleurs, elle est aussi ce qui conduit, peu à peu, cet Ailleurs à ressembler inexorablement à l'Ici. En effet, avec l'extension rapide des routes vers l'Est - à Bayezid, Ella Maillart peut mesurer qu'en deux ans l'ingénieur «Adnan avait avancé de cent quarante kilomètres » (p. 96) - il semble bien que le continent européen gagne du terrain. Avec l'extension du chemin de fer, on dirait même que l'Europe repousse toujours plus vers l'Est sa frontière avec l'Asie. «Maintenant que le rail transanatolien arrive de l'Ouest jusqu'à Erzeroum, il est très probable que Téhéran deviendra le point terminus du réseau sud-est de l'Europe!» (p. 96). D'ailleurs, pour aborder le continent asiatique, il ne suffit plus de passer le Bosphore, ni de traverser la Mer Noire, mais, plus à l'Est, au-delà de l'Iran industrialisé, il faut franchir la frontière qui sépare ce dernier de l'Afghanistan. Ainsi, le chapitre qui relate ce franchissement - comme le chapitre « Routes » - ne porte pas, lui non plus, de nom géographique : il s'intitule simplement « La frontière », comme si, bien que les deux voyageuses aient déjà, à plusieurs reprises, dû remplir des formalités de douane, ce n'était qu'en arrivant en Afghanistan qu'elles passaient véritablement $l a$ frontière, - qu'elles quittaient enfin l'Occident pour entrer en Orient. 


\subsection{Prométhée}

Ella Maillart n'oppose pas l'Occident à l'Orient ; on ne trouve en elle ni rejet des cultures occidentales ni véritable préférence pour les cultures orientales. Ce n'est pas l'Europe qu'Ella Maillart réprouve mais, avant tout, ce qui a fait sortir l'Europe de la voie qu'elle suivait de concert avec l'Asie, et dont la guerre prochaine semble être une ultime conséquence. Or, l'observation des bords de routes, au long du voyage, permet à Ella Maillart de remonter de la guerre à l'industrialisation; et, en remontant davantage encore vers les causes, il semble qu'elle trouve enfin la racine du mal dans un désir prométhéen de dépasser les limites établies par la Nature.

En traversant l'Iran, Ella Maillart comprend comment la guerre peut être favorisée par l'essor du chemin de fer. Sur un ton journalistique, elle analyse en effet comment la formation d'« une armée forte » est le fruit du «développement des transports » joint à « la peur de voisins trop forts $»$ :

Je pense que ce rapide développement des transports [...] caractérise notre époque avec la peur de voisins trop forts. Ce développement [...] a pour premier effet de renforcer l'État et d'augmenter ses revenus. Ces derniers servent tout d'abord à former une armée forte, bien plus importante que ne l'exige l'ordre intérieur. L'armée est une nécessité parce que des voisins puissants, connus pour avoir plus d'une fois débordé leurs frontières, peuvent d'un moment à l'autre trouver le prétexte nécessaire à une promenade envahissante... Crainte, crainte de la guerre, tel est le pivot, le mobile central dans tous les pays qui se réveillent - et pas seulement chez eux ! (p. 118)

La « crainte de la guerre » n'est pas d'aujourd'hui ; elle provient d'une longue histoire commune avec «des voisins puissants » qui ont «plus d'une fois débordé leurs frontières ». Cependant, le développement de l'industrie donne à cette « crainte » des moyens inédits de se satisfaire. En effet, le développement des transports, que ce soit le chemin de fer ou la route, la locomotive ou le camion, permet aux États de dégager de nouveaux revenus qu'ils peuvent alors investir dans la création d'une « armée forte ». Ainsi, le développement de l'industrie et des transports n'entraîne pas la guerre, mais il donne à la «crainte de la guerre » les financements qui lui manquait. Le problème ne réside pas tant dans la guerre - elle est aussi vieille que l'humanité - que dans les proportions inhabituelles que l'industrialisation peut lui donner. Ella Maillart souligne même que ce développement permet à l'Iran de former une armée «bien plus importante que ne l'exige l'ordre intérieur ». L'industrialisation permet de donner à la crainte de la guerre une réponse qui va bien au-delà des stricts besoins : or, pour 
Ella Maillart, c'est dans ce dépassement des besoins que réside l'erreur essentielle de l'Occident.

La racine du mal, en effet, l'origine même du développement industriel, réside dans le désir d'outrepasser ses besoins, ou plutôt dans des désirs qui outrepassent les besoins ${ }^{9}$. Ella Maillart y revient plus d'une fois. Que ce soit pour vanter les montagnards d'Asie centrale «que l'esclavage des besoins artificiels n'a pas encore atteints » (p. 23), pour exprimer son attachement au Pays romand où «rien n'est en excès » (p. 35) ou encore à la Méditerranée, «dont l'atmosphère et la culture sont à la mesure de l'homme » (p. 65), il s'agit toujours de vanter un mode de vie qui s'en tienne aux besoins essentiels, et qui sache demeurer dans les strictes limites des capacités humaines, comme elle en trouve l'exemple parmi les paysans afghans. «Tous les gens que nous avions rencontrés ce jour-là étaient agréables ; ils savaient sourire, ils se comportaient en égaux et non comme des épaves. Ils se mouvaient avec aisance dans une vie à leur taille » (p. 193). Qu'est-ce pour les hommes qu' « une vie à leur taille»? Pour Ella Maillart, il semble que la Nature ait justement proportionné les capacités de l'homme à la satisfaction de ses besoins ; que la simple force de ses bras, sans qu'il n'ait besoin de la décupler, lui permette d'assurer sa propre survie, de se nourrir, de se couvrir, de s'abriter. Ella Maillart ne développe pas une vision édénique de la vie paysanne, où la Nature offrirait à l'homme tout ce dont il a besoin sans exiger le moindre effort; au contraire, «bétails, fruits, peaux de caracul, laines, blé et forêts réclament de la patience » (p. 250); cependant, cette patience et ce travail sont à la mesure de l'homme, de ses bras, de ses mains. Il ne s'agit pas de l'oisiveté de l'âge d'or; il s'agit d'une « vie dure mais bien équilibrée de paysan » (p. 249). Une vie à taille humaine, c'est donc une vie où les efforts nécessaires pour obtenir de la Nature la satisfaction des besoins essentiels ne dépasse pas les capacités de l'homme ; et où, inversement, l'homme ne cherche pas à extraire de la Nature plus qu'il n'est

9 Ella Maillart développe une vision qu'on pourrait qualifier de rousseauiste en plaçant à l'origine du développement des sociétés modernes la volonté de dépasser les capacités physiques de l'homme pour satisfaire des désirs qui excèdent ses besoins essentiels. On se souvient en effet que, pour Rousseau, ce même dépassement détermine un tournant décisif - et funeste - dans l'histoire des hommes : « [...] tant qu'ils ne s'appliquèrent qu'à des ouvrages qu'un seul pouvoit faire, et qu'à des arts qui n'avaient pas besoin du concours de plusieurs mains, ils vécurent libres, sains, bons, et heureux autant qu'ils pouvaient l'être par leur Nature : mais dès l'instant qu'un homme eut besoin du secours d'un autre; dès qu'on s'apperçut qu'il étoit utile à un seul d'avoir des provisions pour deux, l'égalité disparut, la propriété s'introduisit, le travail dévint nécessaire, et les vastes forêts se changèrent en des Campagnes riantes qu'il falut arroser de la sueur des hommes, et dans lesquelles on vit bientôt l'esclavage et la misére croître avec les moissons. » (ROUSSEAU, 1969 : 101) 
utile pour assurer sa survie. Un équilibre est ainsi maintenu entre l'homme et son milieu, où les capacités de l'un sont justement proportionnées aux efforts que l'autre exige pour la satisfaction des besoins essentiels.

Cependant, force est de constater que l'Europe a rompu cet équilibre ; les sociétés européennes, pour satisfaire des désirs excédant leurs besoins essentiels - pour satisfaire des «besoins artificiels » (p. 23) - ont décuplé les capacités humaines. Les machines et le travail ouvrier ont permis aux hommes d'«augmenter leur production journalière » (p. 23); l'industrie a permis aux sociétés d'augmenter rendements et revenus. « Ainsi, petit à petit, on atteint le genre de développement qui caractérise l'Europe et dont l'effet principal sur le reste du monde se résume à ceci : davantage de machines et davantage d'ouvriers spécialisés produisant chaque jour davantage » (p. 249). Or, en dépassant les limites qui lui étaient assignées par ses besoins et ses capacités, l'homme européen a rompu le pacte qui le liait à la Nature. En effet, alors que l'homme et la Nature vivaient dans une sorte d'équilibre, où le premier ne cherchait pas à tirer de l'autre plus qu'il n'était nécessaire à sa subsistance, il s'est mis à exiger d'elle davantage, s'en rendant maître, faisant d'elle son esclave : «tel Prométhée, les hommes se sont attribués tous les pouvoirs de la nature » (p. 179). Or, en comparant l'homme à Prométhée, Ella Maillart souligne non seulement le caractère aventureux de sa révolte, mais aussi sa nature sacrilège, sous-entendant que cette prise de pouvoir ne saurait être sans appeler un châtiment. En s'attribuant «tous les pouvoirs de la nature », les Européens ont rompu un pacte sacré ; contrairement aux montagnards afghans au « mode de vie patriarcal, simple et harmonieux », ils ignorent désormais «un facteur inconnu, appelé "divin" » (p. 179), qu'une punition non moins divine risque néanmoins de leur rappeler.

Et, en effet, si les hommes, grâce aux machines, ont vu dans un premier temps croître leur puissance, Ella Maillart montre aussi combien l'instrument de leur puissance s'est finalement retourné contre eux.

Lorsque l'homme est maître d'une machine, il se sent plus puissant qu'à l'ordinaire, qu'il enfourche une rapide bicyclette ou qu'il gravisse des tours de nuages dans les cieux. [...] Mais quand l'homme est l'esclave d'une machine qui lui dicte les gestes à faire, il découvre bientôt que la vie a perdu toute espèce de goût, et cela bien qu'un meilleur salaire mette à sa portée une nourriture plus abondante et plus savoureuse. (p. 248-249)

Si l'homme s'est rendu maître de la Nature, les machines se sont à leur tour rendu maîtres des hommes: tout d'abord «maître d'une machine», l'homme s'en retrouve finalement «l'esclave». Et l'industrialisation a fini par transformer «de solides paysans ou 
des bergers indépendants en des robots déracinés et amorphes » (p. 249). Il est vrai qu'un meilleur revenu met désormais «à sa portée une nourriture plus abondante et plus savoureuse »; mais au vu des conséquences, la satisfaction de ces besoins excédant les limites du strict nécessaire semble bien chèrement payée. Et même si, à l'échelle non plus des individus, mais d'une société, les revenus dégagés par l'industrie ont aussi permis de fournir «des écoles, des hôpitaux, de la quinine » (p. 249), les deux voyageuses sont néanmoins d'accord pour admettre que les bénéfices tirés de l'éducation ou de la santé ne sauraient compenser les préjudices. Elles le comprennent d'autant plus lorsque, dans la vallée du Pouli-Khumri, elle voient des paysans afghans, en lesquels Ella Maillart trouve par ailleurs un modèle de vie «équilibrée », soumis aux cadences du travail ouvrier. «La question pourrait se résumer ainsi : les avantages que procurent l'hôpital, l'école, le journal ou la radio compensent-ils, aux yeux de l'ouvrier afghan, la perte de ce sourire facile qui accompagnait sa vie dure mais bien équilibrée de paysan ?» (p. 252). Le prix à payer pour certains «avantages »- ou la peine infligée à Prométhée pour s'être emparé du pouvoir - se révèle donc exorbitant, surtout quand «l'harmonie, la joie d'être » cèdent la place à « la dépression morale qui rampe dans le sillage de notre culture matérialiste » (p. 250).

Or, ce que constatent aussi les deux voyageuses, c'est qu'il semble tout aussi impossible de revenir en arrière - «en Europe nous ne pouvons pas faire marche arrière et retourner au système patriarcal où le clan veillait aux besoins de tous ses membres » (p. 249) - que de freiner l'expansion vers l'Asie de ce mode de vie. En effet, tout en comprenant mieux l'origine du mal en le voyant à l'œuvre sur des paysans afghans, elles n'en mesurent pas moins toute l'extension géographique.

Ce n'est pas la modestie de nos savants de laboratoire, l'abnégation de nos recherches, l'honnêteté de nos artisans qui sont connus à l'étranger; mais notre manière de gagner de l'argent, nos machines à coudre, nos métiers à tisser, nos montres, fusils, movies et manuels abrégés. Pourquoi notre civilisation mine-t-elle, sape-t-elle, corrode-t-elle tout ce qu'elle touche ? Pourquoi la plupart des Arabes, Japonais, Hindous ou Chinois adoptent-ils le pire de ce que nous offrons? (p. 251)

S’identifiant ici fortement aux Européens par la répétition des possessifs «nos » et «notre », Ella Maillart craint qu'un seul modèle s'impose au reste du monde, qu'une même «civilisation», fondée sur la satisfaction de besoins artificiels, soit adoptée par «Arabes, Japonais, Hindous ou Chinois », faisant disparaître la variété de leurs cultures et les condamnant eux aussi «au cabanon» (p. 179). Ainsi retrouve-t-on la crainte qu'Ella Maillart 
exprimait à propos des routes, et de leur rôle dans l'expansion vers l'Est de l'industrialisation et des idées occidentales. Il semble bien que la malédiction de l'Europe, son "amère expérience matérialiste » (p. 250), soit appelée à s'étendre, qu'elle soit aussi contagieuse que corrosive; et que le malheur des Prométhée européens n'empêche pas, à son tour, la multiplication en Asie de «petits Prométhée » (p. 252).

\subsection{Déluges}

Devant le caractère irréversible de la civilisation mécanisée et son irrépressible expansion vers l'Est, force est de reconnaître qu'il vaudrait mieux, au lieu de chercher un remède, essayer de tout reprendre à zéro. Aussi, devant l'ampleur du mal - et surtout devant son caractère prométhéen et sacrilège - il semble qu'Ella Maillart soit tentée d'appeler les eaux ou, selon d'autres traditions, les neiges - d'un nouveau Déluge, qui permette au monde de prendre un nouveau départ. Aussi, retrouve-t-on à l'œuvre dans La Voie cruelle deux mythes opposés et complémentaires : d'une part l'Éden, qui représente l'état originel du monde - que certains peuples éloignés, croit-on, n'ont jamais eu l'imprudence de quitter ; et le Déluge qui, au contraire, en marque la fin, dans l'espoir qu'il prenne un nouveau commencement.

Le Déluge biblique est explicitement évoqué quand Ella Maillart traverse en Arménie et en Azerbaïdjan la région qui, selon la tradition, lui aurait servi de décor. Et quand, sur la route entre Erzeroum et Bayezid, apparaît à l'horizon le mont Ararat, elle rappelle que c'est l'endroit «où l'Arche est supposée s'être arrêtée après le déluge» (p. 93). Elle précise d'ailleurs que « sur son versant nord, au couvent d'Etchmiadzine, on montre une pièce de bois provenant prétendument de l'arche. On dit aussi que Noé est enseveli près de là, à Naksivan, la ville la plus vieille du monde $»\left(\right.$ p. 106) ${ }^{10}$.

Or, près de Marand, cette même région servit aussi de théâtre à la version zoroastrienne du déluge dans laquelle, comme le rappelle Ella Maillart,

\footnotetext{
${ }^{10}$ Marco Polo en faisait déjà mention dans sa Description du monde : «Sachez que c'est dans cette Grande Arménie qu'est l'arche de Noé sur une grande montagne »(POLO, 1998 : 79).
} 
le Créateur Ahura-Mazdâ dit au bon berger Yima : «Beau Yima, fils de Vivanhat! Voici que sur le monde des corps vont fondre les hivers de malheur, apportant le froid dur et destructeur. Voici que sur le monde des corps vont fondre les hivers de malheur qui feront neiger la neige à gros flocons, à l'épaisseur d'une aredvi sur les montagnes les plus hautes. (p. 107-108)

Dans cette version du déluge, Yima joue le rôle de Noé, et c'est le dieu Ahura-Mazdâ qui lui annonce que le monde sera bientôt détruit par l'hiver : ce ne sont pas les eaux qui, dans cette version, recouvrent la terre, s'élevant de quinze coudées au-dessus des plus hauts sommets, mais la neige, qui dépasse de «l'épaisseur d'une aredvi» les montagnes les plus élevées. Le dieu ordonne alors à Yima de construire non pas une arche, mais « une résidence souterraine, le Var de Yima, où tous les spécimens vivants vivraient jusqu'au moment d'ouvrir les portes du Var et de repeupler la terre »(p. 108). C'est donc à la blancheur des flocons que la tradition zoroastrienne attribue la fonction d'effacer toute corruption de la surface du monde, pendant que des spécimens de chaque être vivant attendent dans un refuge souterrain non pas le retrait des eaux mais la fonte des neiges.

Or, à voir le monde qui l'entoure, Ella Maillart ne peut que constater l'échec du déluge, qu'il s'agisse de sa version biblique ou zoroastrienne. En effet, les déluges ne sont pas seulement destructeurs : ils doivent surtout purifier ; ils ne marquent pas seulement la fin du monde, ils en inaugurent aussi la rédemption. Dans la Genèse, c'est la corruption des hommes qui conduit Dieu à répandre les eaux du déluge ; c'est parce que « la malice des hommes était extrême, et que toutes les pensées de leur cœur étaient en tout temps appliquée au mal » (Genèse, VI, 5), que Dieu décide de répandre les eaux. Aussi est-ce moins pour détruire l'homme que pour effacer toute trace de corruption que Dieu provoque le déluge, sauvant par ailleurs Noé et sa famille, ainsi qu'un couple de chaque espèce animale, pour donner une seconde chance à sa Création. Cependant, autant la guerre imminente que le «progrès » ou la «civilisation de masses» sont pour Ella Maillart les témoignages de la «malice» des hommes ; ce sont les preuves flagrantes que les aventures de Yima et de Noé ont échoué, et que, contrairement aux desseins de leurs Créateurs, les hommes du XXe siècle sont aussi pervertis qu'avant les neiges des « hivers de malheur » ou les eaux du déluge.

Pourtant, le dieu Ahura-Mazdâ avait bien recommandé à Yima de veiller à ce qu'aucun homme corrompu, tant dans son esprit que dans sa chair, n'entrât dans le Var souterrain : «ni méchant ni trompeur ni rancunier ni jaloux; ni hommes aux dents mal faites, ni lépreux qu'il faut isoler ». 
Il est dit que Yima suivit ces instructions. En voyant la perversité de notre monde, je me demande ce qui a bien pu arriver. Le Créateur a-t-il pu être trompé et Yima a-t-il commis une faute ? Étant peut-être trop gentil, le bon Yima a-t-il laissé entrer dans le Var son meilleur ami et celui-ci, ayant une dent gâtée, est-il devenu jaloux de Yima en sorte que le mal prit naissance une fois de plus? (p. 108)

Méditant par ailleurs l'échec comparable de Noé, Ella Maillart se demande s'il ne serait pas souhaitable qu'un nouveau déluge s'abatte sur le monde.

Lorsque Noé descendit ces pentes où la terre « était encore mouillée et molle du déluge », quel beau jour brillait pour l'humanité renaissante : une vie neuve commençait sur une page sans bavure! Cela se reproduira-t-il ? Devrons-nous tous disparaître à notre tour à l'exception d'un seul qui essaiera de réussir mieux que Noé ? (p. 95)

Or, il semble bien que, dès le début du récit, le nouveau déluge qu'Ella Maillart appelle de ses vœux soit imminent.

Tout d'abord, on se souvient qu'avant leur départ, par la fenêtre d'une maison paysanne, Kini et Christina observent l'Engadine enneigée : «la vallée sans ombre et comme morte », le «lac recouvert de nombreuses couches de neiges », l' « austère désolation », un paysage entièrement dominé par l'hiver ; ce qu'Ella Maillart résume en écrivant : «l'hiver était roi » (p. 21). Or l'évocation au chapitre 10 du déluge de neige, dans la version zoroastrienne du mythe, permet rétrospectivement de mesurer toute la charge dramatique contenue dans cette description initiale d'un paysage hivernal. En effet, il semble bien que ce soit l'un de ces « hivers de malheur» dont parle Ahura-Mazdâ au berger Yima qui ait établi son règne sur les Alpes, comme si Ella Maillart avait souhaité, dès le début, placer son récit sous le signe des déluges.

De plus, si l'épisode de la Genèse est omniprésent par son décor et ses vestiges, il semble bien que les nuages fassent aussi planer au-dessus des voyageuses la menace constante d'un nouveau déluge. Les premières pluies les accompagnent dès la Yougoslavie.

Nous étions à peine entrées en Yougoslavie qu'une pluie rageuse vint fouetter les verdures épaisses des collines, nous forçant a passer la nuit dans un village au bord de la Save enflée. [...] Des inondations avaient emporté la route plus au sud, à ce que l'on disait. (p. 43-44)

L'Arménie est quant à elle noyée sous des orages « hors de saison », à tel point que «les Arméniens avaient peur pour leur moissons » (p. 93). Et, tandis que les voyageuses approchent d'un poste de police, à Taslitschai, « un nouvel orage digne de l'Arche rampait le 
long de notre vallée » (p. 94). Plus loin encore, «vers le nord-est et bien détachée dans la vaste plaine, on pouvait maintenant voir la base conique de l'Ararat flanqué du Petit Ararat ; mais d'humides et denses nuages en voilaient le sommet » (p. 95).

Enfin, après d'aussi nombreux signes avant-coureurs, le déluge s'abat sur le monde quand les deux voyageuses arrivent à Makou :

Nous avions à peine atteint l'auberge que, d'un ciel qui semblait bleu, surgit l'orage le plus violent que j'aie jamais vu jusqu'ici. La nuit tomba sur la terre. L'eau n'arrêtait pas de se déverser et de tout marteler. Un réseau d'éclairs ininterrompus illuminait des nuages violets ainsi qu'une nappe d'eau blanche qui tombait en cataracte du sommet de la falaise surplombante. Je ne sais ce qui était le plus effrayant du bruit de ce nouveau déluge ou de la vue de cette cascade claire tombant d'une hauteur de six cents mètres sur le village obscur entièrement à sa merci. Notre cour était déjà transformée en un réservoir.

Le torrent rageur, les voix qui hurlaient, les coups éclatants du tonnerre, le roulement de l'avalanche, tout se fondait en un rugissement terrible. (p. 103)

Cette description de l'averse qui s'abat sur Makou prend ici le caractère d'une vision biblique, depuis l'orage qui se déclare brutalement au milieu d'un « ciel qui semblait bleu », jusqu'à cette «nappe d'eau blanche qui tombait en cataracte», en passant par «la nuit [qui] tomba sur la terre ». Le texte rassemble en effet plusieurs références à des destructions divines, empruntées au déluge, aux plaies d'Égypte ou encore à l'Apocalypse. Cependant, il est intéressant de noter que l'image de la «cataracte » rappelle non seulement le déluge biblique - où il est écrit que Dieu ouvre «les cataractes du ciel » (Genèse, VII, 11) - mais aussi la destruction du monde dans la tradition zoroastrienne : en effet, la «nappe d'eau blanche » évoque aussi la couleur de la neige, et c'est tout naturellement que, dans la suite du texte, cette «cascade d'eau claire » se transforme finalement en «avalanche ». Sous la plume d'Ella Maillart, l'évocation du déluge prend ainsi tout d'abord la blancheur puis la consistance de la neige, comme si, pour l'imaginaire alpin de la Genevoise, la destruction du monde tendait toujours à prendre le caractère éminemment «plus effrayant» d'une avalanche.

Cependant, rien ne garantit que ce nouveau déluge se montre plus efficace que les précédents. En effet, la leçon qu'Ella Maillart tire des textes religieux, on l'a vu, c'est que, quelles que soient les précautions dont ils se sont entourés, les dieux ont toujours échoué dans leurs œuvres purificatrices. Et, moins encore que les déluges des traditions, il semble que l'averse proprement diluvienne qui s'abat sur le village de Makou aboutisse au renouveau qu'on pouvait espérer. 
En effet, le lendemain, force est de constater que le déluge n'a pas opéré l'action attendue.

À la clarté du jour nouveau, les parois de rocher étaient superbement rouges contre le bleu profond du ciel. Nous escaladâmes le village où, parmi les murs effondrés et les étagères démolies, les gens sauvaient du blé et quelques ustensiles de cuisine. Le maire avait décidé de ne pas reconstruire les maisons sur leur emplacement, mais dans le fond du vallon, près de la route. Ainsi, le ciel aidait le village à s'adapter à une nouvelle époque. (p. 104)

Le jour qui suit un déluge n'est pas seulement le jour suivant, c'est avant tout un « jour nouveau » : il marque un nouveau commencement. Et les deux amies constatent tout d'abord qu'il a comme ravivé les couleurs du monde, le rouge de la pierre contrastant « superbement » contre le bleu du ciel. Cependant, la «nouvelle époque » qui débute n’est pas ce qu'on pourrait appeler un re-commencement : tout ne va pas reprendre à zéro. Il ne s'élèvera pas un nouveau village sur les ruines de l'ancien ; au contraire, le maire profite de cette destruction pour déplacer le village vers le vallon où l'appelait la route. Il semble bien que le déluge, au lieu d'arrêter la marche du progrès, en ait au contraire accélérer le processus. Et on ne peut lire sous la plume d'Ella Maillart sans en sentir toute l'ironie que « le ciel aidait le village à s'adapter à une nouvelle époque ».

Sans doute faut-il penser que, à Makou comme dans le mythe zoroastrien du déluge, un élément corrompu s'est glissé dans l'auberge où, comme Noé dans son Arche ou Yima dans le Var, Kini et Christina avaient trouvé refuge tandis que la pluie faisait rage.

Pendant ce temps, pas le moins du monde impressionné par le désastre, le fils de l'aubergiste, armé de mon stylo, écrivait sous ma dictée le français correspondant à sa longue liste de mots persans. La nouvelle route allait amener un courant constant de voyageurs à Makou : il s'agissait d'être prêt à les recevoir. Il était penché sous cette lampe à pétrole près de laquelle un Allemand fort réservé m'avait dit deux ans plus tôt que Hitler était le seul homme d'État ayant le courage de dire la vérité. (p. 108-109)

Alors que Makou semble sur le point de disparaître sous les eaux, "le fils de l'aubergiste » pense à l'avenir. Bientôt, une «nouvelle route» drainera un flot continu de voyageurs dans son auberge et, pour mieux les servir, il traduit en français « une longue liste de mots persans ». Pour écrire, il s'est installé sous la lampe qui, deux ans auparavant, au même endroit, éclairait un Allemand ouvertement hitlérien, Ella Maillart plaçant ainsi sous le même éclairage - celui du progrès ? de la guerre ? - le jeune supporter de la «nouvelle 
route » et le partisan du nazisme. Or, si le déluge qui se déchaîne au dehors est voué, d'emblée, à l'échec, c'est sans doute parce que, sous les figures du «jeune aubergiste armé de son stylo » et de l'«Allemand»-du moins, de son souvenir - les germes d'un avenir corrompu ont trouvé refuge dans l'auberge, comme dans le Var de Yima, selon la tradition, était entré par mégarde un homme à la dent pourrie. Aussi, une fois les eaux descendues, il n'est pas étonnant que «la nouvelle route » puisse croître de plus belle, détournant ainsi le déluge de sa fonction purificatrice, pour en faire au contraire un instrument du progrès.

Les déluges, qu'ils appartiennent aux traditions biblique ou zoroastrienne, sont donc voués à l'échec. Cependant, pour le voyageur, le déluge peut prendre une autre forme que celle d'une pluie diluvienne. Et, faute de pouvoir ramener le monde à ses origines, il peut encore espérer que le voyage le ramène aux origines du monde - qu'il lui permette de retrouver le chemin de l'Éden. Il ne s'agit pas, ici, de revenir sur l'espace du voyage qui, depuis l'Antiquité, place en Asie le Paradis perdu ; mais de montrer comment le déplacement géographique se dote, pour les deux voyageuses, de vertus proprement diluviennes : comme si le monde, à mesure qu'elles progressaient vers l'Est, devenait de plus en plus originel.

C'est près du mont Ararat, où l'Arche de Noé, dit-on, s'est échouée, que la collusion entre le déluge et le voyage se perçoit avec évidence.

L'Ararat était encore visible et sur son flanc sombre brillaient çà et là les paillettes d'une eau courante. Nous sentions fortement que nous étions à la fin d'un monde: un monde nouveau était sûrement proche de nous. D'ailleurs, c'est quand on a vu Makou - la réplique de Bayezid dans un défilé menant en Iran - qu'on apprécie pleinement ces deux forteresses. (p. 99)

Le mont Ararat semble encore scintiller des «paillettes » du déluge fraîchement retiré. Et il semble bien que la «fin d'un monde » soit proche, qu'un «nouveau monde » soit sur le point de commencer. Cependant, pour le voyageur, le monde qui finit c'est aussi, et avant tout, celui qu'il laisse derrière lui ; et le monde qui commence, celui sur lequel il s'apprête à mettre le pied. Pour le voyageur, l'expression « fin d'un monde » peut aussi bien signifier la destruction d'un univers que le passage d'une frontière. Et justement, la région que traversent les deux voyageuses n'est pas seulement celle du déluge, c'est aussi l'endroit où l'on passe d'une culture à une autre. Aussi les forteresses de Bayezid et de Makou témoignent-elles, quand on les visite successivement, qu'on est effectivement passé d'un monde à un autre. 
Makou est une frontière naturelle. Au-delà, plus de nuages, plus de pluie, plus de montagnes mais un pays ensoleillé, ouvert, fertile parfois, aux lentes ondulations. Finis les caractère latins adoptés par la langue turque ; voici les élégantes envolées de l'alphabet arabe. (p. 105)

Makou est « une frontière naturelle », qui marque une rupture aussi bien dans le climat que dans la végétation; cependant, elle marque aussi un changement de langue - ou plutôt d'alphabet. Et, sous ce point de vue, Makou est une «frontière non plus géographique mais culturelle » (HARTOG, $2001:$ 135) : on quitte le monde turc pour entrer dans le monde arabe. Ella Maillart marque d'ailleurs fortement ce passage en énumérant tout ce qui, à cet endroit, prend fin : «finis les caractères latins », «finis les bœufs et les carrioles », « finis les femmes en costume national, les hommes avec d'amples fonds de pantalon », «plus une seule maison de pierre », « vous pouvez dire adieu à votre vie privée » (p. 105). Pour Ella Maillart, le voyage est ainsi doté d'une fonction diluvienne : le déplacement géographique met fin au monde que l'on quitte aussi radicalement qu'une pluie de quarante jours et quarante nuits. Et le voyageur passe une frontière comme Noé franchit le seuil de son Arche : l'un comme l'autre posent le pied sur un monde nouveau.

On comprend mieux alors l'attention aiguë qu'Ella Maillart accorde aux changements du paysage. «Il importait avant tout de voyager intelligemment, en remarquant les transformations qui différencient une contrée de la suivante » (p. 49). On comprend, aussi, toute l'importance accordée au passage des frontières : aussi bien celles que marquent les postes douaniers et le rituel du passeport, que les frontières naturelles que sont les cols, tel le col du Simplon, «entre pays de plaines et hautes régions, entre l'Europe méridionale et centrale, entre le charme de la chaude latinité et la lourdeur de la réserve germanique » (p. 28).

Le renouveau, d'ailleurs, d'une frontière à l'autre, n'est pas uniquement réservé au monde extérieur. Le voyageur lui-même, en passant une frontière, peut avoir le sentiment d'une rupture plus intime. «C'est commencer une vie nouvelle que de quitter le quai de Galata à bord du vapeur Ankara. Avec notre continent, notre passé et nos erreurs semblaient disparaître derrière nous pour toujours » (p. 70). Aussi, n’est-ce pas un simple déplacement que le voyage réserve aux deux aventurières. En quittant un continent - en quittant leur continent - c'est aussi leur « passé » qu'elles abandonnent ; plus radicalement, en s'éloignant du continent et de leur vie passée, c'est l'un et l'autre qu'elles font «disparaître ». Voyager, 
c'est commencer une nouvelle vie, ou plutôt c'est re-commencer sa vie ${ }^{11}$. Cependant, pour les deux voyageuses, c'est aussi l'occasion d'effacer leurs « erreurs ». Pour elles, le voyage est une façon d'obtenir la rémission de ses fautes - de vivre une sorte de déluge intérieur qui effacerait les erreurs et accorderait la rédemption.

Ainsi Ella Maillart découvre-t-elle que si le déluge ne produit pas, à l'extérieur, le renouveau escompté, par contre le voyage peut provoquer, à l'intérieur, un renouveau limité à la personne du voyageur. Or, comme nous le verrons, c'est à ce changement du dedans qu'Ella Maillart finira par se ranger.

Par-delà la diversité des cultures, il y a une unité de civilisation entre l'Orient et l'Occident : millénaire, cette civilisation s'est répandue de l'Est vers l'Ouest, au rythme lent des caravanes et des invasions. C'est ce que révèlent, le long de la route, les comparaisons possibles entre les modes de vie occidentaux et orientaux. Cependant, comme le constate Ella Maillart en Iran, une nouvelle civilisation, issue de l'Europe, suit à son époque le même chemin, mais en sens inverse : partie de l'Ouest, elle se diffuse le long des routes de l'Est, et cette fois-ci, à la vitesse des locomotives, des voitures et des camions. Or, si, comme nous le verrons plus loin, les invasions anciennes, venues de l'Est, conservaient les cultures des peuples envahis, et même les renforçaient, «les invasions européennes modernes », venues de l'Ouest, qui « ont lieu sous le drapeau de l'humanité et du progrès » (p. 120, citation reprise par Ella Maillart de M. Boveri dans Minaret and Pipeline) ont pour effet d'uniformiser paysages et cultures en propageant une civilisation unique, fondée sur le développement industriel et des besoins artificiels. S'empressant d'aller vers 1'Est, Ella Maillart n'a cependant pas perdu tout espoir : confiante dans les vertus diluviennes du voyage, il semble que ce dernier puisse encore la conduire vers une Europe originelle, préservée des maux de l'Europe actuelle, et la ramener enfin parmi les membres d'une tribu édénique.

${ }^{11}$ C'est une sensation de ce genre que Nicolas Bouvier, au moment de prendre la route, exprime au tout début de L'Usage du monde: «Je pensais aux neuf vies proverbiales du chat; j'avais bien l'impression d'entrer dans la deuxième » (BOUVIER, $2004: 82)$. 


\section{La rencontre de l'Autre}

On peut mesurer l'héritage d'Ella Maillart à son itinéraire, à sa perception de l'espace, ou encore à la localisation de l'Éden en Asie, mais aussi à ce que Tzvetan Todorov, dans Nous et les autres, définit comme la perception exotique de l'Autre et qui, surtout depuis les grandes découvertes du XVIe siècle, caractérise les relations des voyageurs européens. Or, si Ella Maillart est séduite par l'Autre, c'est avant tout par ces signes extérieurs de l'altérité que sont les vêtements traditionnels - comme, en Afghanistan, le turban et le tchador - qui lui permettent d'évaluer la fidélité d'un peuple à sa culture, et de définir dans quelle mesure des traditions peuvent évoluer sans se perdre.

\subsection{La scène de première vue}

La scène dans laquelle, pour la première fois, le voyageur rencontre l'Autre, relève des figures obligées du récit de voyage. Comme la rencontre amoureuse dans les romans, c'est ce qu'on peut appeler une «scène-clé », dont « on ne peut s'empêcher [de] reconnaître le caractère quasi-rituel » et «à laquelle se suspend la chaîne narrative » (ROUSSET, 1984 : 7). Dans Leurs yeux se rencontrèrent, Jean Rousset baptise la «scène-clé » du roman - le moment où deux futurs amants échangent un premier regard - du nom de «scène de première vue » : or, c'est aussi le nom que Jean de Léry donne à son premier contact avec les Indiens du Brésil, dans le sixième chapitre de son Histoire ${ }^{12}$.

Conscient de l'attente de son lecteur, l'auteur d'un récit de voyage peut alors retarder ou, au contraire, anticiper cette scène. Jean de Léry, par exemple, mesurant la curiosité de ses lecteurs par l'impatience qu'il éprouva lui-même de rencontrer des «Sauvages », leur en donne un avant-goût dès le chapitre VI, même s'il se « reserve à les descrire et depeindre au long en autre lieu plus propre », au chapitre VIII (LÉRY, 1994 : 149). Dans La Voie cruelle, Ella Maillart annonce dès les premières pages une rencontre future avec des «hommes libres » (p. 23), mais l'attente du lecteur n'est satisfaite qu'au chapitre 19, à l'instant où les voyageuses voient pour la première fois deux Afghans qui, contrairement aux Iraniens trop européanisés, sont de vraies figures de l'Autre.

\footnotetext{
12 «Du descouvrement et premiere veüe que nous eusmes, tant de l'Inde Occidentale ou terre du Bresil, que des Sauvages habitans en icelle » (LÉRY, $1580: 146)$.
} 
Cette rencontre s'étend de la page 185 à la page 187. Il s'agit du moment où, ayant franchi la frontière afghane, Kini et Christina sont arrêtées par deux douaniers qui les mettent en joue. Cette scène peut être découpée en trois moments - la séduction, l'identification et, finalement, la distanciation - qui correspondent chacun à un mouvement du voyageur vers l'Autre.

La séduction. La «scène de première vue », dans le roman, et la scène de premier contact, dans le récit de voyage, sont comparables non seulement par leur rôle-clé et leur caractère de moment attendu, mais aussi en ce qu'elles mettent en jeu le désir.

Tout à coup, il fallut freiner : deux hommes - turban blanc, dents blanches, ample tunique blanche qu'un gilet ajustait à la taille, pantalon bouffant aux plis profonds - faisaient le geste de nous mettre en joue. Le moment et surtout leur attitude composaient une si parfaite introduction à l'Afghanistan qu'en riant je m'exclamai :

- Je vous l'avais bien dit, ne sont-ils pas splendides?

Nous stoppâmes à leur hauteur. Un soldat - car c'étaient des soldats gardesfrontières que personne n'avait sanglés dans des uniformes kaki, Dieu merci - s'accroupit entre le radiateur et le pare-boue. Ayant rampé comme un chat sur notre dossier et sans pour cela lâcher son arme, l'autre se laissa tomber d'un bloc entre Christina et moi... et nous repartîmes.

Deux tournants à angle droit indiquaient la frontière exacte. Dans ma triomphante allégresse, je déclamais tout ce qui me passait par la tête. [...] (p. 185)

Ce début de la scène rappelle doublement un coup de foudre : tout d'abord, interrompant le voyage dans son mouvement, elle en présente la soudaineté. D'autre part, elle en a aussi les effets euphorisants : à la vue des deux hommes qui les tiennent en joue, la réaction d'Ella Maillart n'est pas de peur, mais d'«allégresse ». Aveuglée par la splendeur des soldats, elle ne voit pas la menace des fusils. Or, pourquoi ces soldats sont-ils si « splendides »? Qu'est-ce qui en fait le caractère séduisant ? Ce n'est pas tant qu'ils soient des hommes ; c'est avant tout qu'ils composent une «parfaite introduction à l'Afghanistan ». En effet, ces hommes attestent doublement l'entrée des voyageuses dans le monde afghan : par leur fonction de «gardes-frontières », mais surtout par leur apparence. Celle-ci se caractérise, d'une part, par sa blancheur : «turban blanc, dents blanches, ample tunique blanche »; or, cette blancheur - qu'on trouve aussi sous le «poitrail blanc » des oiseaux (p. 184) - révèle la pureté du monde afghan et des hommes qui l'habitent, et montre qu'ils n'ont pas encore été entachés par l'Occident. Mais Ella Maillart éprouve une satisfaction encore plus grande à constater que «personne [ne les] avait encore sanglés dans des uniformes 
kaki ». En effet, non seulement, le blanc remplace le vert militaire, mais surtout, le « turban », l'«ample tunique », le «gilet», le «pantalon bouffant aux plis profonds » remplacent les « uniformes »: le particularisme du costume afghan remplace ici l'uniformité de la tenue militaire. Or, si même les soldats portent le vêtement traditionnel, c'est qu'on est vraiment entré dans un pays fidèle à ses traditions. Et pour Ella Maillart, c'est cette fidélité aux coutumes, cette authenticité, qui rend les hommes afghans si attrayants et «splendides ». C'est avant tout leur altérité qui la séduit, la différence de sexe venant renforcer l'attraction produite par une différence avant tout culturelle. Comme Tzvetan Todorov a pu le remarquer à propos des récits de Pierre Loti, «l'étranger est érotique », a fortiori lorsque le voyageur et l'Autre ne sont pas de même sexe et que «la différence des pays coïncide donc avec la différence des sexes » (TODOROV, 1989 : 417).

Les rencontres postérieures entre nos voyeuses européennes et des hommes afghans mêlent d'ailleurs l'altérité culturelle et l'altérité sexuelle, sans qu'il soit toujours possible de les distinguer ou d'attribuer à la première une priorité chronologique. Par exemple, le portrait de cet homme «si beau, faucille en main, yeux dorés sous le turban noir, petite moustache, lèvres pulpeuses montrant des dents saines »(p. 257) mêle des détails du visage et du vêtement, montrant que l'attrait vient à la fois des traits physiques et de différences culturelles visibles. Cependant, comme le précise aussitôt Ella Maillart, «cet Afghan était peut-être capable de voler ou tuer selon un code de l'honneur différent du nôtre, mais avant tout il était homme, un chef-d'œuvre qui rayonnait». Aussi, bien que l'Afghan puisse régler son comportement sur un autre «code», il n'en est pas moins un «homme »: au-delà des différences culturelles, la voyageuse apprécie en lui un «chef d'œuvre » universel de beauté masculine.

Le même jour, une rencontre avec «un paysan solitaire» confirme le caractère érotique de la rencontre avec l'Autre sans insister cette fois-ci sur les différences culturelles :

Non seulement cet homme était beau physiquement, mais ses yeux brillaient d'une belle manière. Cette journée fut illuminée par sa rencontre. Le même éclat vit dans le regard d'une adolescente lorsqu'elle découvre que l'amour l'habite, un amour qu'elle sent si inépuisable qu'il devrait pouvoir transformer le monde tout entier. (p. 257-258)

Tout se joue, ici, dans un échange de regards. Christina et Kini ont fixé leur attention sur un paysan «physiquement beau », et dont elles observent que les « yeux brillaient d'une belle manière ». Au regard séduit des voyageuses, répond ainsi le regard lumineux de l'Autre. Il semble bien, alors, que l'œil du paysan transmette son éclat au regard des voyageuses, 
puisque, brusquement, « la journée fut illuminée par sa rencontre ». Or, cet éclat, capable de passer d'un regard à l'autre, et dont la lumière peut «transformer le monde tout entier », Ella Maillart en trouve l'équivalent dans «le regard d'une adolescente lorsqu'elle découvre que l'amour l'habite». Aussi, la rencontre de voyage non seulement fonctionne, comme la rencontre amoureuse, par un échange de regards, mais elle produit aussi sur le monde les mêmes effets transformateurs. La rencontre de l'Autre amène le voyageur à projeter sur le monde un regard amoureux, comme si le désir éveillé par l'Autre se projetait sur tout ce que le voyageur regarde.

Aussi, il semble que, au cours des rencontres, l'attrait des Afghans, tout d'abord vestimentaire et culturel, devienne de plus en plus physique. Il ne faudrait pas en conclure, cependant, que l'attrait pour une différence apparemment culturelle cacherait, dans le fond, une attirance sexuelle - car des hommes culturellement moins éloignés, en Europe ou en Iran, n'éveillent pas le même intérêt chez les deux voyageuses ; mais plutôt, il semble que, peu à peu, les deux voyageuses prennent la mesure du caractère sexuel qui sous-tend leur attrait pour les différences culturelles. La différence est érotique ; l'Autre est désirable.

L'identification. D'ailleurs, le discours que Kini, juste après leur rencontre avec les gardesfrontières, tient à Christina sur les Afghans, insiste sur les différences culturelles, en montrant combien ces derniers, contrairement aux Iraniens, ont su préserver leurs traditions.

Prenez-y garde, quoique le désert soit ici en tout point semblable à celui de l'Iran, quoique racialement un Mechhedi soit semblable à un Herati, nous venons de franchir une vraie frontière séparant deux pays bien différents. Deux manières de vivre - réciproquement méprisées - semblent être à l'origine de ce contraste. Le reis iranien nous prenait en pitié parce que nous allions voyager parmi ces «sauvages Afghans»... et je vous parie mon appareil de cinéma que le chef afghan parlera comme il y a deux ans de « ces rampants Iraniens avec lesquels il vaut mieux n'avoir rien à faire»! (p. 185)

Ella Maillart sait bien que les vraies différences ne sont ni dans les paysages, ni dans les visages ; et que l'apparente uniformité d'un « désert », voire même d'une race, peut en fait dissimuler « une vraie frontière séparant deux pays bien différents ». Les vraies différences ne sont pas dans la nature - du paysage, des hommes - mais dans les cultures : les «manières de vivre ». La «vraie frontière », pour reprendre la distinction de François Hartog, est moins géographique que culturelle. La voyageuse sait aussi qu'entre peuples voisins, les différences culturelles peuvent être «réciproquement méprisées»: l'un parlera des «sauvages Afghans »; l'autre, de «ces rampants Iraniens». Ella Maillart prend soin, d'ailleurs, 
d'attribuer ces discours nationalistes, respectivement, au «reis iranien » et au « chef afghan », et de les rapporter entre guillemets. Cependant, elle ne prend pas les mêmes précautions lorsque, plus avant dans ce même discours, elle compare femmes iraniennes et afghanes : «Ici, pas une seule gourgandine à la mode iranienne en robe courte et portant talons trop hauts : vous êtes dans le pays sans femmes, où des hommes coiffés de neigeuses mousseline portent de gros souliers cloutés en forme de gondole» (p. 186). Qui qualifie, ici, la femme iranienne de «gourgandine »? Il semble bien que l'on soit toujours en présence du discours nationaliste afghan - et, d'ailleurs, les talons que portent ces femmes ne peuvent paraître «trop hauts » que par rapport à une norme afghane, où les femmes sont soumises à des restrictions vestimentaires. Cependant, ici, Ella Maillart n'attribue ce discours à aucun émetteur, et aucun guillemet n'en manifeste la nature de citation. Il semble, au contraire, que la voyageuse reprenne à son compte le discours des Afghans sur les Iraniennes, qu'elle en adopte le point de vue. En quelque sorte, par le discours, Ella Maillart prend la place de l'Afghan : elle s'identifie à l'Autre. Tout se passe comme si la voyageuse, en franchissant la frontière, avait aussi franchi la distance culturelle qui la sépare de l'Autre. Comme si elle était devenue autre.

La distanciation. Ainsi, Ella Maillart franchit-elle - en paroles du moins - le pas qui sépare la séduction de l'identification. L'attirance qu'elle éprouve pour l'Autre la porte tout naturellement à s'assimiler à lui. Or, ce discours assimilateur est interrompu par le silence et les gestes du douanier afghan, qui contraignent bientôt la voyageuse à reprendre sa place :

Je ne pouvais pas consacrer mon attention à mes effets d'éloquence : j'étais constamment distraite par notre nouveau voisin et par ce qui devait sans doute se passer dans sa tête. Il ne pouvait comprendre qui était ces deux personnes étrangères qui se noyaient dans un flot de paroles. L'automobile avait peut-être été volée de l'autre côté de la frontière ? Était-il possible que ces deux personnes fussent des femmes ?

La douce lumière du tableau de bord n'éclairait que la partie inférieure de nos mentons minces et féminins. Mais il y avait des cheveux courts sur nos têtes. Les tentatives de conversation n'ayant apporté aucun éclaircissement, l'Afghan décida de résoudre le mystère selon les moyens à sa disposition: très lentement, simultanément, ses mains suivaient la courbe de nos côtes ! Est-il possible d'imaginer situation plus ridicule? Nous ne pouvions pas nous permettre d'être offensées et de nous faire des ennemis de ces Afghans félins, car nous étions somme toute à leur merci. Explications ou injonctions étaient inutiles. Réprimant notre humeur folâtre, nous espérions que notre attitude gênée leur ferait comprendre nos sentiments. (p. 186-187) 
Contrastant avec le discours plein «d'éloquence » et «le flot de paroles » par lesquels Ella Maillart donne libre cours à l'enthousiasme provoqué par la rencontre, le soldat qui s'est glissé entre les deux voyageuses garde le silence. Or, Ella Maillart n'oublie pas totalement cette présence, car de même que les deux Afghans sont pour les voyageuses un spectacle exotique, de même, elles sont pour eux un objet de curiosité. Le mutisme du douanier contraint Ella Maillart à imaginer «ce qui devait se passer dans sa tête », et à se percevoir, elle et Christina, à travers le regard de l'Afghan, dont non seulement elle imagine les interrogations, mais dont elle adopte aussi le point de vue : « la douce lumière du tableau de bord n'éclairait que la partie inférieure de nos mentons minces et féminins ». Ainsi, non seulement, «les voyageurs deviennent les sujets du regard de l'autre » (FORSDICK, 2008 : 60), mais ce même regard conduit le voyageur à percevoir sa propre altérité. Or, pour un Afghan, l'altérité des étrangères tient à leur apparente androgynie : dans ce pays où les femmes ne montrent pas leur visage et ne portent pas les cheveux courts, il ne saurait déterminer par de seuls signes extérieurs le sexe de ces deux «personnes » aux mentons féminins, mais à la coupe de cheveu masculine.

D'ailleurs ce n'est pas la première fois que la méprise sur les deux voyageuses les conduit à prendre la mesure de leur propre altérité. Des enfants turcs les confondent avec des Allemandes (p. 61) ; «un écolier géorgien » prend Christina pour le « fils » de Kini (p. 99$100)$; et, plus d'une fois au long de la route, Christina est prise pour un homme. «Plus nous allions vers l'Est, et plus elle était fréquemment prise pour un garçon » (p. 71). Plus Christina avance vers l'Autre, plus sa propre altérité devient évidente. Cependant, ces regards ne sont pas seulement l'expression d'une erreur de jugement : ils sont révélateurs de l'étrangeté des femmes européennes à l'aune de critères orientaux, qu'il s'agisse de leur apparence (port du pantalon, coupe des cheveux), ou que, comme Christina, elles puissent être épouse sans être mère :

Apprenant qu'elle était mariée depuis plusieurs années, le maire demanda où étaient ses enfants. Lorsqu'il sut qu'elle n'en avait pas, il s'exclama :

- Mais alors qu'avez-vous fait pendant tout ce temps ? (p. 223)

Aussi, dans la scène de première vue, face à l'androgynie des deux Européennes, le soldat afghan doit-il substituer au simple regard un geste qui tient, tout à la fois, de l'exploration et de la caresse, et qui manifeste plus fortement encore le mélange de curiosité et de désir qu'inspire la différence : «très lentement, simultanément, ses mains suivaient la courbe de nos côtes !». À cet instant, les deux Afghans «splendides » deviennent des 
« félins », tenant les voyageuses «à leur merci », - et de simple spectacle qu'on admire et qu'on commente, ils deviennent une réalité bien plus tangible et menaçante. Or, c'est au moment où le soldat passe du regard à la caresse, et du désir à sa réalisation, que les deux femmes prennent enfin conscience du caractère érotique de la rencontre ; et que, réprimant leur propre «humeur folâtre », elles «prennent une attitude gênée ». C'est à ce moment, aussi, que le douanier afghan non seulement ramène les voyageuses à leur identité de femmes, mais qu'il leur rappelle leur identité d'Européennes.

Plus loin, d'ailleurs, un regard d'hommes afghans rappelle à Ella Maillart ce double aspect de son identité. «Nous nous éloignâmes. Nous attirions trop l'attention ; et pour ma part, j'aurais aimé que ma jupe fût plus longue » (p. 202). Le regard de l'Autre, à nouveau, portant sur une particularité vestimentaire des femmes européennes - la «jupe » - contraint non seulement Ella Maillart à prendre conscience de sa différence tout à la fois culturelle et sexuelle, c'est-à-dire de son altérité, mais aussi à s'éloigner, et donc à rétablir la distance qui la sépare de l'Autre et qu'elle avait cru possible d'abolir.

Ainsi, la scène de première vue qui relate, en même temps, le passage d'une frontière et le premier contact avec des Afghans, est entièrement fondée sur le regard et la distance. En effet, la vue de l'Autre, dont le caractère exotique se double d'un attrait érotique, incite le voyageur à réduire la distance qui l'en sépare. Ce qu'il parvient à faire, du moins par le discours, en prenant le point de vue de l'Autre, en adoptant ses valeurs - au détriment, ici, d'un tiers (les Iraniennes). Cependant, le regard de l'Autre rappelle bientôt au voyageur sa propre altérité, culturelle et (dans La Voie cruelle) sexuelle, le contraignant à rétablir la distance qu'il avait cru possible de réduire. La même altérité qui l'avait séduit, et à laquelle il s'était identifié, est finalement ce qui contraint le voyageur à reprendre sa place initiale.

Aussi, trouve-t-on en germe, dès cette scène de première vue, l'échec de la rencontre : le regard de l'Autre révèle au voyageur son irréductible altérité au monde auquel il aspire. Le franchissement d'un poste frontière ne suffit pas à relever toutes les barrières : au passage d'une frontière géographique ne correspond pas forcément le franchissement d'une frontière culturelle. 


\subsection{Le turban}

La séduction des soldats afghans, on l'a vu, provient avant tout d'un costume traditionnel qui témoigne d'une victoire du particulier sur l'uniforme: on pourrait tout aussi bien dire de l'identité sur l'informe. Pour Ella Maillart, en effet, le costume montre que l'Afghan, contrairement à l'Européen et à l'Iranien, a su rester lui-même. Or, plus encore que la différence, c'est cette fidélité à soi-même qui la séduit. Dès lors, la description des vêtements ne remplit pas seulement, comme dans tout récit de voyage, une fonction ethnographique : elle mesure un degré de fidélité à soi-même, d'authenticité.

Parmi les vêtements afghans, l'attention d'Ella Maillart se porte avec prédilection sur le turban, qu'elle compare à la «casquette» iranienne pour en faire un symbole de l'authenticité afghane :

En regard de l'Iran où le turban a été interdit, l'Afghanistan où il reste en faveur mérite une mention spéciale : étant donné qu'on est influencé par ce qu'on porte, la tête des Afghans est fièrement droite. Puisque, en entourant son crâne d'une longue cotonnade, l'homme gagne en dignité et en beauté, ne serait-il pas un idiot d'adopter la misérable casquette de l'Iranien ? Même le simple calot qui sert de base au turban est plaisant lorsqu'il est brodé de couleurs vives. En voyant le couvre-chef de l'Iranien d'aujourd'hui, on ne s'étonne plus que cet homme ait l'air honteux de lui-même! (p. 202)

Il est intéressant de voir comment Ella Maillart passe ici de l'observation ethnographique d'une coutume vestimentaire à des considérations d'ordre moral. Elle détaille en effet assez précisément la posture à laquelle « la longue cotonnade » contraint celui qui porte un turban : or, par cette posture, l'homme "gagne en beauté et en dignité ». De la tête droite à la droiture, il n'y a qu'un pas, qu'Ella Maillart franchit au nom d'une règle qu'elle formule ainsi : «on est influencé par ce qu'on porte ». Le vêtement a, pour la voyageuse, une influence directe sur la moralité d'un peuple. Ainsi, la faveur dans laquelle l'Afghan a maintenu le «turban » est-il garant d'une fierté nationale; au contraire, l'Iranien, qui l'a troqué pour la «casquette », a non seulement perdu la posture droite, mais a pris en échange un «air honteux de lui-même». Or, qui parle de cet «air honteux», ou de «la misérable casquette de l'Iranien »? Qui sous-entend que ce dernier est bien « idiot» d'avoir abandonné le turban ? Est-ce Ella Maillart ? Sont-ce les Afghans ? Il semble bien que le discours ne fasse pas seulement entendre ici la voix de l'écrivain, mais qu'au style indirect libre, il reprenne aussi la voix de nationalistes afghans. Cependant, rien dans l'énoncé, aucun guillemets, ne 
permet de vraiment dissocier la voix d'Ella Maillart de celle des Afghans, comme si, de nouveau, par le discours, elle s'identifiait à ces derniers au détriment de l'Iranien.

On retrouve cette même association d'une tenue vestimentaire et d'une posture morale dans un souvenir qu'Ella Maillart a conservé de son précédent voyage sur cette même route. Même s'il ne s'agit pas, ici, du turban, mais du «foulard de tête » que portent habituellement les Arabes, il est toujours question d'un couvre-chef traditionnel.

La plupart de mes compagnons portaient le foulard de tête arabe avec l'écheveau de laine tordue comme serre-tête. Ils s'appelaient mutuellement hadji et avaient donc été à La Mecque. Un homme au visage marqué de variole et qui parlait anglais était tailleur à Hamadhan. Son frère - l'un des mollahs de Mechhed - avait été outré de voir comme il s'était européanisé : il était rasé de près et portait un élégant pull-over bleu sur une chemise de tennis dont les pans étaient cachés dans le pantalon à la mode occidentale. (p. 163)

Il ne s'agit pas, ici, de comparer les Iraniens et les Afghans, mais, parmi les Iraniens, de distinguer ceux qui portent «le foulard de tête arabe » et ceux qui s'habillent «à la mode occidentale ». D'emblée, Ella Maillart s'associe clairement aux premiers, qu'elle appelle «mes compagnons » et dont elle comprend parfaitement les expressions en langue arabe. Or, leur foulard n'est que le signe extérieur d'une fidélité plus ample à leurs traditions : en effet, s'ils s'appellent « hadji » et sont allés en pèlerinage à la Mecque, c'est qu'ils respectent aussi les traditions religieuses. Au contraire, le «tailleur » originaire d'Hamadhan, qui a troqué la langue arabe pour «l'anglais » et porte les pans de sa chemise «dans le pantalon à la mode occidentale », marque une distance à l'égard de ses traditions, qui n'est pas, elle non plus, uniquement vestimentaire, puisqu'elle lui vaut les réprobations de son frère, «l'un des mollahs de Mechhed ». Or, la faute commise par ce tailleur, c'est de s'être « européanisé », et l'insistance d'Ella Maillart sur son « visage marqué de variole » tendrait même à rapprocher cet européanisation d'une maladie : le tailleur, en quelque sorte, a été contaminé par l'Europe.

En Afghanistan, de même, Ella Maillart distingue les Afghans qui ont préservé leurs traditions de ceux qui ont adopté la mode européenne. Après avoir franchi la frontière, un même épisode se répète deux fois : la Ford s'enlise dans les sables. La première fois, les deux voyageuses sont secourues par « quatre robustes montagnards » :

Avec leurs turbans noirs, leurs gilets brodés sur des habits de lin, le blanc de leurs yeux lourdement entourés d'antimoine, ils ressemblaient à des brigands. Lorsqu'ils eurent replacé l'automobile sur la route, Christina mit un billet de cinq afghanis dans la main du plus grand. Il regarda le billet, leva 
les yeux vers elle, rendit l'argent et partit sans avoir dit un mot. Je n'ai jamais vu yeux et contenance plus fiers que ceux-là. (p. 192)

$\mathrm{Au}$ second enlisement, les voyageuses sont aidées par des «hommes de basses extraction et peu plaisants » dont l'un « gémissait et soupirait sans interruption ».

\begin{abstract}
Imaginez-le dans une chaleur approchant 44 degrés, les jambes serrées par des jarretelles et les pieds dans des souliers de ville en cuir jaune, tandis que son corps jutait sous un complet de serge noire ! Lorsqu'il tomba la veste et les bretelles pour nous aider à sortir du sable, nous vîmes une chemise européenne à larges rayures violettes, vertes et roses. Arrogant avec les inférieurs, servile avec ses supérieurs, il était laid - et cela dans un pays où le plus pauvre des paysans est digne d'admiration. (p. 229)
\end{abstract}

Dans les deux cas, à nouveau, les tenues vestimentaires sont mises en relation avec des attitudes morales. D'un côté, ceux que les voyageuses prennent tout d'abord pour des brigands, portant «turbans noirs » et « gilets brodés », se révèlent en fait d'un profond mépris pour l'argent, affirmant ainsi leur «contenance » et leur fierté. De l'autre, au contraire, un homme vêtu d'une «chemise européenne » se montre «arrogant » et « servile », témoignant d'une laideur morale que redouble sa laideur physique.

Aussi, quand, à propos du turban, Ella Maillart prend parti pour les Afghans contre les Iraniens, elle ne marque pas une préférence pour la culture des uns contre celle des autres. Elle valorise la tendance des uns à conserver leur culture, et stigmatise la tendance des autres à adopter le mode de vie européen. Sur le plan vestimentaire, les habits européens sont des indices du même type que, au niveau du paysage, les marques du progrès industriel : les uns et les autres témoignent de la progressive expansion de la civilisation européenne vers l'Orient. Aussi trouve-t-on, d'une part, des peuples qui, par leurs vêtements, montrent qu'ils ont conservé leurs traditions et leur dignité ; et ceux dont, au contraire, les vêtements sont les signes extérieurs d'une européanisation - d'une contamination par l'Europe - et d'un avilissement moral.

Le vêtement permet ainsi de mesurer la fidélité d'un individu à sa culture, et surtout l'éloignement ou le rapprochement avec l'Européen, Ella Maillart valorisant toujours celui qui s'en éloigne le plus - auquel elle s'identifie, qu'il soit Iranien ou Afghan - au détriment de celui qui s'en rapproche. Or, cette valorisation par le voyageur du lointain géographique, 
au détriment du proche, est caractéristique de ce que Todorov, dans Nous et les autres, appelle l'« exotisme ».

La perception exotique propose, selon Todorov, une représentation de l'Autre diamétralement opposée à la perception ethnocentrique ou nationaliste. Pour mieux caractériser l'attitude nationaliste à l'égard de l'altérité, Todorov rappelle l'exemple des Perses qui «parmi les autres peuples », selon Hérodote, « estiment d'abord, après eux-mêmes toutefois, leurs voisins immédiats, puis les voisins de ceux-là, et ainsi de suite selon la distance qui les en sépare, les peuples situés le plus loin de chez eux sont à leurs yeux les moins estimables ». Ainsi, dans la perspective nationaliste, la valeur d'un peuple dépend de sa distance géographique : plus il est proche, plus il est estimable ; plus il est éloigné, plus il est méprisable.

On peut donner un autre exemple de ce que Todorov appelle la « règle d'Hérodote » (TODOROV, 1989 : 239), en rappelant comment les Grecs eux-mêmes, toujours selon l'auteur des Histoires, estimaient les peuples du nord :

[...] la sauvagerie va croissant plus on s'avance en direction du nord et du nord-est. Cette dernière proposition n'est vraie que globalement; mais le mouvement marque bien une diminution de la part d'humanité, jusqu'à ce qu'on atteigne ces êtres, seulement connus par ouï-dire, que sont les hommes aux pieds de chèvres, les Arimaspes et les Griffons. (HARTOG, 2001 : 7172)

Dans ce cas, il ne s'agit plus seulement de mesurer la valeur d'un peuple selon son éloignement, mais aussi son degré d' «humanité » et de «sauvagerie ». Les Grecs représentant une humanité civilisée, les peuples du nord correspondent à des degrés d'humanité de plus en plus faibles en fonction de leur distance géographique et, inversement, de sauvagerie de plus en plus élevés. Ainsi, à mesure que l'on s'éloigne du monde hellénique vers le nord, on rencontre des peuples de plus en plus sauvages, jusqu'à trouver, aux marges du monde connu, des êtres monstrueux, mi-humains, mi-animaux, les degrés les plus éloignés de l'humanité tendant à se confondre avec l'animalité.

Or, dans la perception non plus nationaliste mais exotique de l'Autre, cette échelle de valeurs s'inverse: l'Autre s'y trouve d'autant plus valorisé qu'il est éloigné géographiquement, une humanité idéale - et non plus monstrueuse - devant forcément se trouver aux frontières du monde connu. On trouve un exemple de cette attitude dans L'Odyssée, où «Homère évoque en effet les Abioi, alors population la plus éloignée parmi celle que connaissent les Grecs, et les déclare "les plus justes des hommes" » (TODOROV, 
1989 : 356) ; et Todorov propose de baptiser cette attitude, par opposition à la « règle d'Hérodote », du nom de « règle d'Homère ».

Les attitudes relevant de l'exotisme seraient donc le premier exemple où l'autre est systématiquement préféré au même. Mais la manière dont on se trouve amené, dans l'abstrait, à définir l'exotisme, indique qu'il s'agit ici moins d'une valorisation de l'autre que d'une critique de soi, et moins de la description d'un réel que de la formulation d'un idéal. (TODOROV, 1989 : 355)

La perception exotique de l'Autre, en effet, implique non seulement une valorisation de ce qui est lointain, mais aussi une dépréciation de ce qui est proche, l'éloge de l'Autre n'étant en fait bien souvent qu'une critique plus ou moins dissimulée de soi. Dans l'Histoire d'un voyage faict en la terre du Brésil de Jean de Léry, l'Autre lointain - l'Indien du Brésil, le Tupinamba - est d'autant plus célébré qu'il incarne une figure antithétique de l'Européen ; il est d'une santé d'autant plus robuste, que l'Européen est rongé de maladie ; il est d'autant plus pur, que l'Européen est corrompu. Et toute description élogieuse du Tupinamba se double alors d'une critique de l'Européen.

Dans La Voie cruelle, nous retrouvons cet exotisme hérité des voyageurs du XVIe siècle, dans lequel s'articulent l'éloge du lointain et la critique du proche. Alors que l'Afghan, coiffé du turban, tient sa tête «fièrement droite», Ella Maillart s'interrogeant sur la décadence européenne se demande : «Quand avons-nous cessé [...] de porter la tête droite ?» (p. 262). Ainsi, le lointain Afghan, pour Ella Maillart, incarne le degré d'humanité le plus noble, et son compatriote européen, au contraire, le plus dégradé - l'Iranien, situé à michemin, représentant alors le chaînon intermédiaire.

Cependant, si la perception exotique compare les peuples en fonction de leur éloignement géographique, l'exotisme d'Ella Maillart compare plutôt les peuples en fonction de leur éloignement culturel : de leur degré de résistance à l'européanisation et d'attachement à leur propre culture. En effet, pour Ella Maillart, il ne saurait être question de comparer les cultures européenne, iranienne ou afghane, mais de vérifier - en particulier par le vêtement en quoi chacune des sociétés concernées se montre fidèle à sa propre culture. Si l'Européen représente le plus bas degré de l'échelle, c'est moins à cause de sa proximité, que de son absence de culture : en effet l'Européen et, à son exemple, d'autres sociétés, ont renoncé à leurs cultures au profit d'un «matérialisme qui n'a pas de quoi les remplacer» (p. 262); l'Iranien occupe sur l'échelle une position intermédiaire, dans la mesure où, en phase d'européanisation, il n'a pas encore perdu sa culture, mais se voit contaminé peu à peu par un 
modèle étranger ; quant à l'Afghan, à l'abri de ses frontières naturelles et, surtout, de sa religion - «attaché d'autre part à l'Islam, il ne peut prêter qu'une oreille distraite aux raisonnements athées de ses voisins nordiques » (p. 212) - il semble qu'il soit demeurer fidèle à son identité afghane.

Ainsi, Ella Maillart ne valorise pas en l'Afghan une culture, mais un peuple plus fidèle à sa culture, un peuple résistant aux changements, et qui sait préserver l'héritage du passé.

Ici où le mode de vie n'a pas encore changé, où le fils pense comme le père, les hommes ont gardé leur dignité d'homme. Tandis qu'en Occident, où tout n'est que changements, personne ne sait que penser, personne ne sent son avenir assuré - les riches moins que quiconque - et cela même durant les périodes de paix. (p. 185-186)

La supériorité de l'Afghan, sa « dignité d'homme », ne lui vient pas de caractéristiques propres à son identité culturelle, mais de son attachement inébranlable à son passé. En effet, l'homme qui répète les gestes de son père, qui lui-même les tenait de ses ancêtres, ne connait pas l'angoisse de l'avenir : pour savoir de quoi sa vie sera faite, il lui suffit de se rappeler celle de son père ; pour prévoir le futur, il lui suffit de se tourner vers le passé. Pour qui respecte ses traditions, rien ne saurait être différent de ce qui a été. Au contraire, l'infériorité de l'Occidental lui vient d'une infidélité à sa culture, dont l'abandon le condamne à vivre non seulement sans passé - il s'en est coupé - mais surtout sans avenir : car, si pour « le fils qui pense comme le père », demain sera pareil à hier, au contraire, pour le fils qui renonce à l'héritage paternel, aucun passé ne saurait plus donner la moindre idée de l'avenir. Aussi, la crainte d'Ella Maillart, c'est que l'Afghan puisse un jour lui aussi renoncer à son passé, qu'à l'exemple de son voisin européanisé, il ne soit «contaminé par la maladie iranienne de démolir tout ce qui paraît ancien » (p. 188). Pour la voyageuse, le bien réside dans la continuité, et le malheur, dans la «rupture avec le passé » (p. 250). Conséquence de l'industrialisation, la discontinuité est le vrai malheur de l'Occident.

\subsection{Le tchador}

Le vêtement traditionnel témoigne donc de la fidélité de celui qui le porte à ses traditions, il est le signe d'un attachement à sa culture - le signe d'une fierté culturelle. Cependant, le prix qu'Ella Maillart accorde aux traditions, et le mépris qu'elle manifeste pour l'adoption du mode de vie occidental, n'impliquent pas forcément le refus de toute évolution des cultures. 
Et l'analyse du comportement d'Ella Maillart à l'égard d'un autre vêtement afghan, le tchador (voir annexe 3), peut aider à nuancer sa position à l'égard des changements culturels.

De même que le turban amène Ella Maillart à comparer les Iraniens et les Afghans, de même, le tchador la conduit tout d'abord à comparer les femmes iraniennes aux femmes afghanes. D'un côté, on l'a vu, elle critique ces « gourgandines à la mode iranienne en robe courte et portant talons trop hauts » (p. 186), reprenant à son compte un discours afghan pour mieux stigmatiser l'occidentalisation des femmes d'Iran qui se montrent trop ; d'autre part, elle souligne qu'en Afghanistan, où la femme au contraire se montre le moins possible, « vous êtes dans un pays sans femmes », sans qu'elle n'y voie, tout d'abord, rien à reprendre. Ainsi, le goût d'Ella Maillart pour la fidélité aux traditions la conduit non seulement à tenir un discours nationaliste contre les Iraniennes, mais encore à reprendre un discours masculin contre les femmes. L'exaltation des traditions amène ainsi une femme européenne à adopter un discours d'homme afghan.

Or, plus loin, elle semble apporter un premier bémol à cet éloge des traditions afghanes prononcé dans l'enthousiasme de l'arrivée.

Nous croisions parfois quelques-unes de ces femmes « dissimulées »-
silhouettes en linceul guidant leur pas grâce au petit «guichet» ou treillis
brodé devant leurs yeux. Lorsqu'on est en automobile, elles sont un danger
public car elles ne voient presque rien et entendent encore moins (la mode
demande que le «linceul » serre la tête et les oreilles de très près). Ce n'est
que lorsqu'on arrive à leur hauteur qu'elles sautent de côté, tout époulaillées.
(p. 202-203)

Ici, le voile que portent les femmes, contrairement au turban que portent les hommes, ne fait pas l'objet d'une admiration mais d'une irritation - voire d'un dénigrement. La voyageuse en automobile s'irrite de ces femmes portant le tchador qui traversent devant elle. Il y a inadéquation entre un transport moderne et un vêtement traditionnel ; cependant, ce n'est pas la voiture qui est un «danger public », mais le vêtement qui empêche de voir et d'entendre. Ce n'est pas le moyen de transport qu'il faudrait changer, mais la tenue vestimentaire. De plus, si Ella Maillart se montre capable, dans d'autres passages, d'adopter le regard de l'Autre pour critiquer la mode occidentale, ici, elle reprend au contraire un point de vue européen pour juger «la mode» de l'Autre. D'une part, elle utilise l'adjectif «époulaillées » pour assimiler les Afghanes à des volailles qui, au passage d'une voiture, se disperseraient au tout dernier moment : or, cet adjectif appartient au patois savoyard, et le 
choix d'un terme aussi régional montre combien, à cet instant, Ella Maillart revient à ses origines pour tourner l'Autre en ridicule. D'autre part, l'image du « linceul » non seulement porte un jugement sur le tchador qui rend les femmes aveugles et sourdes au monde des vivants, mais surtout, exprime elle aussi un point de vue européen sur le vêtement afghan. En effet, on retrouve cette même image, un peu plus loin, dans les paroles d'un ingénieur allemand qui marque ainsi son rejet des traditions afghanes : «Il n'était pas prêt à cacher sa femme sous un linceul chaque fois qu'elle devrait sortir de la maison » (p. 246). Or, en rapportant ici les paroles de l'ingénieur au style indirect libre, Ella Maillart semble les reprendre à son compte, et s'en montrer solidaire.

On pourrait accuser Ella Maillart de se contredire, de louer les traditions dans l'enthousiasme de l'arrivée, et de les critiquer ensuite dans un moment d'irritation. Mais ce serait ignorer que l'identité du voyageur est multiple et que, selon les circonstances, Ella Maillart peut réagir en ethnologue, en Occidentale, en Suissesse, ou en femme. Or, si l'ethnologue peut faire l'éloge d'un « pays sans femmes », la femme, elle, peut critiquer le cloisonnement des Afghanes. De plus, ce serait donner à la position d'Ella Maillart envers les traditions un caractère trop rigide. Or, qu'elle se permette de critiquer certaines coutumes nous conduit à nuancer cette position : en effet, si Ella Maillart peut se montrer nationaliste ce nationalisme à l'envers, qui prend le point de vue de l'Autre, et que Todorov appelle l'exotisme - elle n'en est pas pour autant intégriste : c'est à dire qu'elle admet, à l'intérieur des traditions, la possibilité - voire même, parfois, la nécessité - de changements.

C'est ce que montre la rencontre, un peu plus loin, des deux voyageuses avec « une femme voilée qui marchait seule, d'une manière décidée et chaussée de talons hauts »- où, curieusement, le voile afghan semble s'accommoder des mêmes «talons hauts » que portent les Iraniennes.

En arrivant à sa hauteur, nous vîmes qu'elle avait relevé le devant de son tchador gris : émacié, avec des yeux pâles et inflexibles, son visage était européen, ce visage qui, pour un instant, vivait à l'air libre ! Elle cessa de se voiler lorsqu'elle vit que nous étions des femmes. (p. 283)

Ici, la «femme voilée » n'est plus perçue avec ce regard éloigné qui sépare le voyageur d'une coutume étrangère : deux éléments permettent au contraire d'abolir la distance. D'une part, les voyageuses reconnaissent dans les traits de l'inconnue un visage « européen »; d'autre part, l'inconnue perçoit que les deux voyageuses sont des «femmes». S'il est surprenant pour Kini et Christina de trouver une Allemande sous un tchador, il ne doit 
pas l'être moins pour cette dernière de trouver deux femmes au volant d'une voiture. Or, l'étonnement passé, le «voile»-dans tous les sens du terme - tombe : la communauté culturelle et sexuelle opère un rapprochement immédiat qui s'exprime par l'exclamation : «ce visage qui, pour un instant, vivait à l'air libre !», dans laquelle deux voix se mêlent : le soulagement de l'Allemande et la compassion d'Ella Maillart ${ }^{13}$.

La suite du texte continue de mêler les deux voix, la narratrice rapportant au style indirect libre les explications de l'inconnue: «Son autocar était devant elle, mais elle préférait aller à pied, car la route était dangereuse. Son mari était afghan et travaillait à Mazari-Sherif. Elle retournait à Kaboul après une très mauvaise crise de malaria » (p. 283). Cependant, le style indirect marquant la solidarité des deux voix s'interrompt, et la distance narrative se rétablit quand, à la fin de la rencontre, le tchador rabattu s'interpose à nouveau entre les voyageuses et l'Allemande : elle n'est plus alors ni européenne, ni même femme, mais redevient l'objet d'un regard extérieur, « une anonyme pyramide de coton avec de petits carrés brodés devant les yeux ». C'est alors la seule voix de la narratrice qui reprend la parole sous la forme exclamative :

Douze ans d'attente patiente avant de pouvoir être libérée de ce domino, avant qu'elle puisse redevenir elle-même et que le vent et le soleil à nouveau caresse sa joue ! Je me félicitais d'avoir mes bras nus offerts au soleil. Nous nous félicitions de notre liberté, si difficile à endurer mais plus nécessaire que la vie. (p. 283)

Exprimés de telle sorte, les «douze ans » tombent comme une sentence de prison : c'est le temps que l'Allemande devra patienter avant d'être « libérée ». De plus, le voile qui l'empêche « de redevenir elle-même », la prive non seulement de sa liberté, mais aussi de son identité. Ella Maillart ne voit plus, dans le vêtement traditionnel, ce qui garantit à l'homme son identité, mais au contraire ce qui l'en prive. Cependant, ce n'est plus l'ethnologue qui s'exprime ici, mais le «je» de la femme, aussitôt amplifié par le «nous » dans lequel se manifeste la solidarité féminine des deux voyageuses. Cette rencontre leur permet alors, par contraste, de mesurer la liberté qu'elles ont acquise par le voyage, en opposant le tchador, qui isole du monde, aux «bras nus » et aux visages amoureusement offerts aux caresses du

\footnotetext{
${ }^{13}$ Ella Maillart peut d'autant mieux compatir que, dans un précédent voyage, elle a déjà porté ce voile : «Après la leçon, les femmes veulent me faire mettre un de leurs voiles et elles rient de tout leur cœur en me voyant suffoquer sous cet objet malpropre » (MAILLART, 2001b : 110).
} 
« vent » et du « soleil » (répété deux fois); un vêtement qui représente la prison et la mort, à la « liberté $[\ldots]$ plus nécessaire que la vie ».

D’évidence, ici, Ella Maillart appelle de ses vœux la fin du voile pour les femmes afghanes, laissant entendre que, dans certaines conditions, les traditions doivent être modifiées. Or, la façon dont l'Allemande voilée rapporte les tentatives entreprises pour supprimer le tchador permet de mieux comprendre dans quelles conditions un tel changement peut être acceptable :

Elle pensait que l'époque approchait où l'on pourrait abandonner le tchador mais ce n'était pas aux quelques femmes européennes mariées à des Afghans d'en donner l'exemple, à moins qu'elles ne tiennent à se rendre la vie impossible ; c'était aux dames alliées au roi Zaher de donner ce signal. En 1929, la femme du roi Amanullah avait commencé une campagne pour la suppression du voile des femmes. Mais cela ne fut pas populaire et n'eut pas de succès, en partie parce que la reine était une Syrienne catholique. (p. 283)

Le tchador peut être supprimé, mais ce n'est pas à des «femmes européennes » d'en prendre l'initiative ; et si la tentative de 1929 a échoué, c'est parce que la promotrice du changement était «une Syrienne catholique », c'est-à-dire doublement étrangère dans un Afghanistan musulman.

Ainsi Ella Maillart nous laisse-t-elle entendre que les traditions peuvent souffrir des changements : mais ce ne peut être, comme en Iran, de ces bouleversements radicaux imposés de l'extérieur, sous l'influence de l'Occident ou la pression de l'Union soviétique ; les traditions ne doivent changer que sous la conduite de ceux qui les vivent et les respectent, comme si, mues par une poussée propre et progressive, elles évoluaient d'elles-mêmes, de l'intérieur.

À propos des changements d'origine extérieure qu'une société peut subir sous l'influence d'une autre, Ella Maillart adopte une attitude que Todorov appelle le relativisme, et qui milite en faveur du maintien des différences et, pour cela, du minimum de contacts entre les peuples. On l'a vu, quoique voyageuse, elle se méfie des moyens de transports qui diffusent les techniques et les idées ; elle rêve de routes qui, comme à l'époque de Marco Polo, séparent plus qu'elles ne relient entre eux des pays éloignés. Des routes qui ne soient pas des lignes de communication. En effet, pour le relativiste, les échanges entre peuples risquent de modifier leur identité d'origine : l'authenticité, la fidélité à ses traditions, sont d'autant plus menacées chez un peuple qu'il multiplie ses contacts avec d'autres. Aux 
contacts, le relativiste préfère l'isolement; au mélange, il préfère l'endémie. En effet, pour le relativiste,

si la communication s'accélère, alors les différences s'estompent et l'on s'avance vers l'universalisation de la culture - c'est-à-dire d'une culture au détriment des autres. [...] Passé un certain seuil, la communication est donc néfaste, car elle conduit à l'homogénéisation, laquelle équivaut à son tour à un arrêt de mort pour l'humanité. (TODOROV, 1989: 107)

Or c'est bien cette « universalisation de la culture » que redoute Ella Maillart, et donc cette «communication » entre cultures qui conduit à la disparition des différences au profit d'une seule civilisation. Ainsi, en Afghanistan, se montre-t-elle opposée à l'introduction d'« innovations surgies de-ci, de-là, non seulement aux fabriques et entreprises industrielles hâtivement mûries, mais aussi aux maisons modernes à larges fenêtres et à toits minces impossible à chauffer au cours du glacial hiver, aux complets étriqués, aux souliers pointus en similicuir, aux bicyclettes » (p. 250) - c'est-à-dire à des produits qui, sous couvert du progrès et de la modernité, sont en fait étrangers. Par contre, elle sera favorable à tous les «produits afghans nécessitant des activités afghanes » qui «ne suscitent pas de rupture avec le passé, pas de développement imposé et forcé » (p. 250). Toute solution intérieure lui semblera toujours préférable à celles qui viennent de l'extérieur.

Aussi, elle adhère aux méthodes réformistes du gouvernement afghan. En Afghanistan, en effet, deux tendances s'opposent : il y a, d'une part, les tenants de la tradition et, de l'autre, ceux de la réforme. «L'Afghanistan se partageait entre Anciens et Modernes. Groupés autour des mollahs, les Anciens s'opposaient à toute innovation. [...] Quant aux audacieux Modernes, qui ont presque tous été formés à l'étranger, ils sont le clan gouvernemental » (p. 295). Or, contrairement à toute attente, Ella Maillart ne prend pas le parti des traditionnalistes, mais bien celui des réformateurs, même si ces derniers, « formés à l'étranger », risqueraient, du point de vue d'un relativiste, de contaminer leur culture en y introduisant les ferments de l'occidentalisation. Mais ici, comme à propos du tchador, la femme parle plus haut que l'ethnologue, en particulier sur la question de l'éducation des filles, à laquelle sa condition féminine la rend particulièrement sensible.

Si les mollahs exigent «que l'on ferme l'école des filles, car une éducation moderne pouvait seulement causer du trouble dans des cervelles féminines » (p. 295), il est d'autant plus clair, ici, qu'Ella Maillart rapporte les propos des religieux musulmans sans y adhérer que, précédemment, en visitant une de ces écoles de jeunes filles, elle avait émis le souhait « qu'on leur apprît à penser, sentir et vivre justement » (p. 294). Et la fierté, la tête droite, qui 
étaient, semble-t-il, le propre des hommes dont les turbans symbolisaient le respect des coutumes, sont au contraire ici le propre des jeunes écolières qui ont abandonné le tchador et, montrant «toutes sortes de visages et d'habillements différents», semblent s'exclamer : «C'est brave ce que nous faisons en étant ici, nous sommes fières d'être le nouvel Afghanistan !» (p. 294). Le nouveau l'emporte, ici, sur l'ancien : il semble qu'Ella Maillart opère une inversion des valeurs.

Mais, c'est qu'ici les « Modernes » n'entrent pas en conflit avec les « Anciens ». Au contraire, le gouvernement afghan a retenu une leçon du passé : c’est que « les réformes trop précipitées d'Amanullah avaient rendu toute la population hostile aux changements ». Aussi savent-ils que «les mollahs doivent être convertis aux nouvelles idées afin d'être renvoyés dans leurs villages comme agents de propagande pour le régime actuel ; en ce moment, il n'y a qu'eux qui puissent fournir les cadres nécessaires pour construire un nouveau pays » (p. 295). La réforme doit passer par les tenants de la tradition ; il ne doit pas y avoir opposition entre les deux, ni rupture : il faut que la réforme soit issue de la tradition, qu'elle la travaille, qu'elle l'infléchisse de l'intérieur. «Les réformes gouvernementales devront se faire en accord avec l'esprit de l'Islam si elles veulent être durables » (p. 280)

Ella Maillart trouve l'incarnation de ce pragmatisme dans une infirmière de Kaboul.

Elle avait appris le Coran afin d'avoir le dernier mot avec ses patients. Même lorsqu'ils souffraient d'une forte fièvre, ils voulaient parfois à tout prix s'agenouiller cinq fois par jour sur les dalles froides au moment de la prière; mais dans le Livre, elle avait trouvé le passage autorisant ceux qui sont couchés à faire leurs prosternations symboliquement en abaissant les paupières. (p. 296)

Plutôt que de renier les enseignements du Coran, l'infirmière les incorpore pour les utiliser au service de la santé de ses patients musulmans. Elle entre ainsi dans leurs croyances pour permettre certains changements d'attitudes tout en demeurant à l'intérieur des traditions.

Cependant, la frontière est souvent ténue entre réforme (intérieure) et bouleversement (extérieur), la première risquant toujours de finalement verser dans le second. Au point que, devant certaines mesures prises par ces gouvernants modernes dont Ella Maillart semble apprécier le tact, elle éprouve malgré tout des inquiétudes : «Hashim Khan, l'intelligent Premier ministre d'Afghanistan, verra-t-il qu'en introduisant trop de méthodes occidentales parmi ses tribus, il va les bouleverser?»(p. 250). Or, force est de constater que, 
contrairement à ce qu'elle pensait, et malgré les précautions des gouvernants, l'Afghanistan n'est pas, lui non plus, épargné par les maux de l'Occident.

En effet, contrastant avec la description d'une longue scène afghane, où Ella Maillart louait « un monde stable qui ne sait rien de la semaine de quarante heures [...], ni rien des rotatives inondant le monde de journaux innombrables »(p. 243), les deux voyageuses découvrent brusquement, au détour de la route, un spectacle diamétralement opposé :

La scène rappelait certaines descriptions du Far West : baraquements, tentes, échoppes, ateliers dans le grand vent de poussière ; partout du bruit et de l'activité, des wagonnets Decauville se pourchassant, des rivets qu'on martelait, des bétonneuses mâchant leur ciment - tout cela remuant, lançant des éclairs, transportant ceci ou cela... Nous étions à Poul-i-Khoumri. (p. 244)

Alors que les deux voyageuses abordent le point le plus à l'Est de leur voyage, il leur semble au contraire être arrivées en plein «Far West». Comme si l'Occident, à force de repousser ses marges toujours plus loin vers l'Ouest, avait débordé l'Amérique et, faisant le tour de la terre, avait finalement abordé l'Asie par l'Est. Comme si, où que l'on soit, à l'Est comme à l'Ouest, l'industrie avait recouvert le monde d'un même paysage et répandu «partout du bruit et de l'activité ».

C'est que les ingénieurs qu'elles pensaient avoir laissés sur des chantiers de l'Iran les ont en fait précédées dans la figure de «Herr Khun »; et qu'avec lui, il a non seulement apporté « le Progrès, notre dieu émacié qui profite des guerres » (p. 248), mais aussi, accroché au mur de leur pavillon « un portrait d'Hitler » qui «dominait la table à café, le chien favori, les coussins et la maîtresse de maison. Étions-nous toujours en Asie ?» (p. 246). Avec le progrès, les ingénieurs introduisent aussi en Afghanistan un mode de vie qui, autant que le paysage industriel, menace de retirer tout caractère asiatique à l'Asie.

Cependant, pour Ella Maillart, le signe le plus frappant du déclin de la culture afghane se lit, une fois de plus, sur les vêtements (voir annexe 4). Et si, en franchissant la frontière, elle avait été séduite par la blancheur des turbans, elle constate que, dans la vallée du Poul-iKhoumri, «il y avait là une grande variété d'hommes, mais tous portaient le même chiffon sale en guise de turban » (p. 244). Non seulement, le monde industriel a souillé le couvre-chef que les Afghans portaient si fièrement, faisant passer le «turban» au rang de simple « chiffon », mais surtout, ce turban qui manifestait leur différence culturelle est désormais ce qui, au contraire, efface les différences parmi la «grande variété des hommes ». Or, c'est dans cette image matérielle d'un turban devenu chiffon sale, qu'on peut mesurer le caractère tout à 
la fois homogénéisant et dégradant de l'occidentalisation ; et que se manifeste clairement chez Ella Maillart l'horreur d'une relativiste devant la disparition des particularités culturelles sous la triste bannière d'une seule et même civilisation. Au point que Poul-i-Khoumri peut devenir «le symbole de notre âge mécanique oppressant le cœur de l'homme, de notre civilisation mécanisée copiée servilement par les pays de l’Asie » (p. 249).

\subsection{Les nomades}

Aussi, même protégé par ses montagnes, l'Afghanistan n'est pas l'Éden attendu : les germes de l'occidentalisation y sont déjà plantés. Et, à propos du barrage que l'on construit à Poul-iKhoumri, Ella Maillart est bien obligée de constater qu' «ici, au cœur de l'Asie », ce cœur de l'Asie vers lequel, depuis ses premiers voyages, elle a toujours orienté ses pas, «ce grand monstre avait pris racine » (p. 244). Cependant, Ella Maillart conserve un dernier espoir de trouver, parmi les nomades qu'elle croise plusieurs fois dans son voyage, un dernier échantillon d'humanité encore indemne. Faute d'Éden, elle espère encore pouvoir rejoindre une «tribu édénique ».

D'une part, il semble que les tribus nomades conservent mieux leurs traditions que les peuples sédentaires, et puissent ainsi mieux résister à l'occidentalisation. En effet, le nomadisme implique que les tribus compensent leur mobilité par la fixité de leurs usages, sous peine de perdre leur identité : aussi sont-ils «fortement eux-mêmes » (p. 276). Les Tziganes, par exemple, qu'Ella Maillart croise à deux reprises ${ }^{14}$, ont une identité si forte que, de l'Europe à l'Asie, ils restent les mêmes et que «le spectacle » qu'ils offrent aux deux voyageuses en Afghanistan leur rappelle « de semblables rencontres sur un autre continent »: «Nous avions vu le frère de leur ours poussiéreux et mangé par les mites deux mois auparavant, en Yougoslavie » (p. 219). Aussi retrouve-t-on chez eux la «dignité » (p. 46), « le caractère indomptable » et ces airs «nobles» (p. 219) qui, pour Ella Maillart, sont l'expression d'une fidélité aux traditions.

\footnotetext{
${ }^{14}$ Une première fois au tout début du voyage, en Yougoslavie : «Au crépuscule, nous nous arrêtâmes près d'un camp de tziganes où la fumée traînait sur les véhicules » (p. 46) ; une seconde fois, à l'autre extrémité du périple, en Afghanistan : «Soudain, je devins alerte, excitée même : une caravane aux couleurs gaies venait tout en désordre vers nous. [...] C'étaient des Jats ou Tziganes allant aux Indes par Kandahar » (p. 218 ).
} 
D'autre part, même si les Tziganes ont une forte identité, ils ne sombrent pas pour autant dans le nationalisme :

\begin{abstract}
Je me demande pourquoi ils m'attirent toujours aussi fortement, même au Turkestan où l'on ne peut dire qu'ils évoquent de lointaines contrées. Est-ce parce qu'ils sont le symbole de ce que j'essayais d'être, dépourvus de biens, partout chez eux, intensément vivants, sans maîtres, sans les limites qu'impose une nationalité ? (Je ne crois pas que des Tziganes allemands et russes pourraient se battre dans les armées nationales de ces pays ; ou bien s'ils le font, c'est qu'ils ont abandonné leurs traditions de vraie indépendance.) Les Français les appellent bohémiens ou romanichels, les Hongrois, tziganes; mais eux-mêmes se nomment simplement Romani, « hommes ». (p. 46)
\end{abstract}

Les Tziganes réalisent cet idéal d'être à la fois «intensément vivants », mais sans connaître «les limites qu'impose une nationalité »; ils peuvent être « partout chez eux », sans pour autant appartenir à aucun pays. Ils ne sont ni «allemands », ni « russes », mais tout simplement «Romani »: autrement dit, des «hommes ». Ella Maillart trouve en eux une sorte d'humanité originelle, une humanité d'avant que les hommes ne se fixent en un lieu, n'établissent des frontières et ne délimitent des pays - une humanité d'avant les nations. Aussi, la distance qui sépare la voyageuse des nomades s'inscrit-elle moins dans l'espace que dans le temps : ils évoquent moins «de lointaines contrées », qu'ils n'incarnent un Paradis perdu. Et l'attirance qu'éprouve Ella Maillart pour cette humanité édénique, relève moins de la curiosité ethnographique que de la nostalgie d'une vie antérieure : «je dois avoir vécu parmi eux jadis... » (p. 45).

Ainsi Ella Maillart voit-elle dans les Tziganes un peuple préservé tout à la fois de l'occidentalisation et du nationalisme, un peuple ayant une culture forte, mais sans pour autant qu'il s'identifie avec une nation - alors qu'en Europe, à la veille de la Seconde Guerre Mondiale, Ella Maillart ne trouve plus au contraire que des nations fortes sans culture. Mais les nomades ne sont pas seulement épargnés des maux de l'Europe ; ils représentent aussi, aux yeux d'Ella Maillart, un remède capable de guérir l'Occident - et les pays occidentalisés, comme l'Iran - de ses maux.

Car les nomades sont le levain qui pourrait régénérer les Syriens abâtardis, les Iraniens épuisés, les Chinois décimés. Dans le passé, après une courte période de dévastation, les nomades infusèrent l'audace de leurs conceptions aux peuples qu'ils avaient conquis. Greffés sur de vieilles souches, ils provoquèrent une nouvelle floraison en Chine, en Iran, en Turquie. Depuis les Aryens d'il y a très longtemps jusqu'aux Arabes, Seldjoukides et 
Mongols, combien plus pauvre serait notre monde sans l'impulsion donnée par ceux qui, comme le roi David, étaient des nomades ? (p. 277)

Aux métaphores de la maladie qui caractérisent souvent l'occidentalisation, Ella Maillart oppose ici celles du «levain » et du greffon. Les deux familles d'images ont en commun l'idée d'une insémination: mais si l'une épuise, décime, l'autre au contraire revigore, rajeunit, refleurit. Alors que l'expansion moderne de l'Europe est perçue comme une épidémie, les invasions nomades du passé sont considérées, malgré la « dévastation » qu'elles ont pu engendrer, comme une «nouvelle floraison ». C'est que la civilisation européenne détruit les cultures sans les remplacer, et que les nomades transmettent « l'audace de leurs conceptions »: ils ne détruisent pas les cultures qu'ils traversent, ils leur donnent une nouvelle «impulsion ». Les nomades agissent comme des catalyseurs de cultures.

Cependant, Ella Maillart constate en Iran que « les nomades et leur manière de vivre avaient disparu» (p.150) et, de la Turquie à la Mongolie, que «la vie nomade est condamnée » (p. 276). Elle montre alors par quel processus les Kurdes, les Turkmènes et les Mongoles disparaissent progressivement. D'une part, «les frontières d'aujourd'hui sont nettement tracées », empêchant les nomades de circuler librement, et les retenant à l'intérieur d'un même pays. D'autre part, « désirant devenir fort, le pouvoir central de chaque pays à besoin de soldats obéissants et de contribuables à domicile fixe »; aussi les gouvernants mettent-ils tout en œuvre pour fixer les nomades enfermés dans leurs frontières. Et finalement, même si certains «ont de la peine à devenir paysans ou artisans », et renâclent devant la vie sédentaire, «leurs enfants seront sans doute plus adaptables puisqu'ils n'auront pas connu l'ancienne vie ». En-cloisonnement, fixation puis sédentarisation : tels sont les trois moments qui ponctuent l'absorption des cultures nomades à l'intérieur d'une nation. Le rapport de force s'est inversé : si autrefois les nomades revigoraient les peuples en s'y greffant, ils sont aujourd'hui détruits par ceux qui les absorbent.

Ainsi, avec la disparition progressive des nomades, il semble que le monde perde ses dernières tribus édéniques, et sa dernière chance de survie. Néanmoins, renonçant à la dimension collective de son voyage, Ella Maillart pourrait chercher son salut individuel, en allant vivre parmi ces derniers nomades auxquels elle se sent si profondément apparentée. Cependant, il semble que tous ses efforts pour les approcher débouchent sur un échec.

Quand elle croise pour la deuxième fois, à Chibargane, une tribu tzigane, Ella Maillart saisit son appareil photo : «Cavaliers, piétons, ours, singes et enfants furent reçus à coups de 
déclics d'appareils»(p. 219). Dans La Voie cruelle, les deux voyageuses ont en effet des appareils photos qu'elles ont toujours peur de perdre, que ce soit à l'occasion d'un contrôle policier (p. 62) ou d'un vol (p. 270). Ella Maillart emporte aussi dans son bagage une caméra dans le but de réaliser un film documentaire sur les tribus nomades ${ }^{15}$. Cependant, ces appareils n'ont pas pour seule fonction d'enregistrer les étapes du voyage et les peuples observés. Comme le rappelle Charles Forsdick, «l'appareil sert souvent de lien entre la voyageuse et ceux qu'elle rencontre » : «Nouvelle technologie légère et portable, l'appareil photo Leica sert souvent de filtre entre la voyageuse et le terrain qu'elle traverse, permettant ainsi un contact avec ses habitants qui serait autrement plus difficile » (FORSDICK, 2008 : 39). Ignorant souvent la langue des pays qu'elle traverse, Ella Maillart utilise l'appareil photo comme un substitut de la parole, un truchement, un instrument non seulement d'enregistrement, mais de communication.

Cependant, lors de sa dernière rencontre avec des nomades, il semble bien que même la caméra ne permette plus à Ella Maillart d'établir un contact :

Je traversai un gué du Ghorband pour filmer des femmes turkmènes en train de planter les souples gaules qui soutenaient leur tente ronde en feutre. Mais elles ne me trouvèrent pas à leur goût. Je cédai. Fermant mon appareil, je les regardai humblement, incapable de leur expliquer que je les aimais et que la vie sous une yourte comme la leur avait été pendant des années le symbole de mes désirs les plus chers. (p. 284)

Le franchissement de la rivière qui sépare la voyageuse des Turkmènes exprime sa volonté d'un rapprochement. Il semble qu'Ella Maillart veuille ainsi franchir une dernière frontière. Cependant, son approche bute contre un refus, auquel Ella Maillart, en cédant, répond à son tour par un renoncement. En «fermant [son] appareil», elle rompt le lien qui l'unissaient aux nomades, et cette rupture de communication se manifeste dans l'incapacité « de leur expliquer»ses sentiments. Au regard médiateur de la caméra succède le regard nu de la voyageuse : les Afghans, on l'a vu, regardent fièrement, mais l'Européenne, ici, regarde « humblement ». Car si les femmes afghanes sont inaccessibles, ce n'est plus une question d'éloignement spatial mais, plus radicalement, de distance ontologique : l'Européenne n'est pas «à leur goût ». Alors la «yourte», au début si matérielle (c'est au film qu'on doit sans doute la description de la tente dont Ella Maillart précise, en quelques mots, la structure, la

\footnotetext{
${ }^{15}$ C'est à Bombay, en septembre 1940, qu'Ella Maillart montera et projettera le film Nomades afghans.
} 
forme et la matière), ne peut plus être qu'un «symbole de [ses] désirs les plus chers »- désirs de vie nomade voués à l'inassouvissement. Et cette dernière tentative de se joindre aux tribus nomades, soldée par un échec, précède immédiatement le retour des deux Européennes à la « civilisation » (p. 287).

Ainsi, l'échec de la rencontre dont la scène de première vue contenait déjà les prémices, devient définitif dans cet ultime essai de rapprochement avec des nomades. Et la guerre qui éclate quand Ella Maillart et Christina sont à Kaboul leur interdit toute autre tentative.

Puis la guerre éclata dans la lointaine Europe.

L'incertitude s'empara de nos vies. [...] Les étrangers n'eurent plus la permission de quitter Kaboul pour l'intérieur du pays. Mon Kafiristan était donc hors d'atteinte. (p. 293)

Ainsi, ce que montre l'Autre, au terme du voyage, c'est qu'il ne suffit pas de quitter l'Europe pour retrouver l'Asie, de laisser derrière soi un monde corrompu pour regagner l'Éden, une humanité déclinante pour revenir vers une humanité originelle. D'une part, l'Asie, même la mieux protégée par ses murailles, ou sa religion, n'est pas épargnée par la civilisation industrielle; d'autre part, l'Autre nous montre qu'on est toujours soi-même : et Ella Maillart n'est jamais plus européenne que sous le regard des nomades afghans auxquels elle aurait tant voulu s'identifier. Ce sont deux raisons qui conduisent alors la voyageuse à rejeter le voyage extérieur, au bénéfice du voyage intérieur, le seul qui puisse peut-être ramener vers cet Éden tant recherché et qu'on ne saurait peut-être trouver ailleurs qu'en soimême. 


\section{Le dehors et le dedans}

L'échec du voyage, l'impossibilité de retrouver l'Éden perdu, ou de se fondre dans une tribu édénique, ramènent l'attention d'Ella Maillart du monde extérieur vers le monde intérieur ou, pour reprendre ses propres termes, $\mathrm{du}$ «monde objectif » vers le «monde subjectif » : vers les tourments de Christina, aux prises avec la drogue, mais aussi vers ses propres interrogations. Cette dimension subjective du récit de voyage, qui n'est plus simple exploration mais se double d'une introspection, amène Ella Maillart à modifier les règles du genre, et à se demander si une autre écriture ne serait pas plus adaptée à saisir ce voyage intérieur.

\subsection{Monde objectif/Monde subjectif}

Au début du récit, Ella Maillart fixe un «double but» à son voyage : «aider [son] amie et arriver à Kaboul » (p. 32). De Kaboul, elle espère pouvoir atteindre la vallée de l'Amou-Daria et rejoindre une tribu nomade dont l'étude lui permettrait de comprendre les maux de l'Europe. Tel est l'objectif extérieur qu'Ella Maillart attribue à son voyage. Mais il est aussi un objectif intérieur: en effet, Christina souffre d'une dépression dont seule la morphine parvient à la soulager, et Kini s'est engagée à guérir sa compagne de route.

Aussi, Ella Maillart voyage en quête de deux remèdes : un premier, qui pourrait sortir l'Europe de la guerre ; un second, qui pourrait sortir Christina de la drogue - l'Europe et Christina pouvant d'ailleurs s'assimiler l'une à l'autre, pour ne faire qu'un, lorsque Kini compare sa compagne de voyage à «ces nations qui veulent la paix et préparent des conférences de désarmement tout en sachant qu'elles ne sont pas prêtes à rendre la guerre impossible» (p. 69). Et le voyage se poursuit sur deux plans : à la «surface de la terre » et « intérieurement », le déplacement géographique se doublant d'un parcours intérieur.

Sur la surface de la terre, là où j'ai déjà voyagé, je retrouverai le chemin qu'il nous faut suivre; et intérieurement, où dès longtemps je me pose des questions semblables aux vôtres, que le peu que j'ai trouvé puisse nous aider à vivre jusqu'à ce que vous trouviez ce qu'il faut trouver par soi-même. (p. 27)

Cependant, ces deux plans ne sont pas indifférents l'un à l'autre ; au contraire, ils sont susceptibles d'interférer : d'une part, le monde extérieur peut influer sur le cours des pensées ; 
d'autre part, et c'est ce qu'Ella Maillart comprend en observant Christina, la vie intérieure peut modifier la perception du monde alentour.

Mentalement, nous étions bien différentes. Elle était un poète se mouvant parmi les idées qui naissaient et vivaient de son imagination, son humeur transformant le monde, tandis que je croyais encore à la réalité des faits en eux-mêmes, persuadée que le monde extérieur conditionnait ma vie subjective. (p. 130)

Le type de rapport qui s'instaure entre le monde et les êtres permet ainsi de déterminer ce qui distingue Christina et Kini : la première, en «poète», vit au milieu de son « imagination »; l'autre, en voyageuse, vit au contraire dans «la réalité des faits ». Mais ce n'est pas tout. En effet, non seulement Christina accorde la priorité à la vie intérieure, mais elle est aussi capable d'agir sur le monde extérieur : son « humeur » transforme «le monde». Elle établit avec le dehors une relation qu'on pourrait qualifier de centrifuge. Au contraire, Kini accorde non seulement la priorité au monde réel, mais elle en fait aussi dépendre sa vie intérieure - le «monde extérieur » conditionne «sa vie subjective »-établissant ainsi avec le dehors une relation centripète. Or, il est intéressant de noter qu'Ella Maillart modalise son propos : «je croyais encore », écrit-elle, «à la réalité des faits », comme si, des deux voyageuses, des deux rapports possibles entre le dedans et le dehors, c'était Christina qui était dans le vrai et Kini dans l'erreur.

L'influence du monde extérieur sur Ella Maillart apparaît tout d'abord, comme nous l'avons déjà vu, quand elle se met à pleurer au milieu de Paris : l'approche palpable de la guerre ne laisse pas la voyageuse indifférente. L'état du monde agit directement sur son état d'esprit. Quitter le continent européen revient alors à vouloir se soustraire à son influence néfaste.

J'étais trop faible ou trop bête pour échapper à la contagion des révoltes, des paniques, des militarismes et de la manie de faire des plans qui enfiévrait l'Europe. La distance me serait une aide, sûrement. En Occident, tout le monde semblait aussi égaré que moi-même; pourquoi ne pas aller vers l'Orient ? (p. 59).

D'ailleurs, on l'a vu, le franchissement des frontières, et tout particulièrement de ces frontières naturelles - à la fois géographiques et culturelles - que sont les cols, produit en Ella Maillart un changement intérieur, comme si «avec notre continent, notre passé et nos erreurs semblaient disparaître pour toujours » (p. 70). Aussi, qu'Ella Maillart soit en Europe ou en voyage, il semble bien que le dehors continue de conditionner le dedans, à la différence que, 
si Europe exerce sur la voyageuse une oppression, l'Asie au contraire apporte une libération, un allègement, une rédemption.

Or, chez Christina, le rapport entre dehors et dedans s'inverse : ce n'est pas, comme pour Ella Maillart, l'état du monde qui fait jaillir les pleurs, mais au contraire son état dépressif qui transforme le monde en «vallée de larmes ».

Comme Christina parlait de sa nuit gâchée par une douleur imaginée, je compris brusquement que le ciel ou l'enfer sont, à chaque instant, la conséquence des pensées que notre manque de connaissance nous suggère. Et quels êtres pitoyables nous sommes de laisser ces projections de notre intellect composer une vallée de larmes ! (p. 125)

Une «douleur imaginée» ne se cantonnent pas aux seules limites de «notre intellect»; au contraire, projetées sur le monde extérieur, nos «pensées » peuvent le transformer en « ciel » ou en « enfer ».

Aussi, dans un premier temps, Ella Maillart essaie d'inverser chez sa compagne de voyage le rapport entre le dehors et le dedans et, loin de l'Europe, d'exposer Christina à l'influence bénéfique de l'Asie. Dans l'espoir de modifier l'humeur dépressive de sa compagne de voyage, « de la distraire d'elle-même» (p. 130), elle lui montre alors la beauté du monde extérieur : «Je soulignais le "cri" d'une fleur rouge contre le bleu du ciel, la joie de chacun de nos pas choisi » (p. 128). Cependant, tous ses efforts se soldent par un échec.

Une fois de plus je m'efforçais de changer le cours des pensées de Christina. Je savais mon effort puéril car je me rendais compte chaque jour davantage combien la nature intérieure conditionne à chaque pas ce qui nous vient de l'extérieur. (p. 128)

Ainsi, l'incapacité d'Ella Maillart à modifier par le spectacle du monde extérieur le cours intérieur des pensées de sa compagne la conduit à revoir ses idées sur l'importance du monde objectif et son impact sur le monde subjectif. Ses difficultés - et finalement, son échec - à guérir Christina de sa dépression conduisent Ella Maillart à revoir ses premières convictions: et si, avant le départ, elle pensait encore que «le monde extérieur conditionnait [sa] vie subjective », le drame de sa compagne lui prouve au contraire que «la nature intérieure conditionne à chaque pas ce qui nous vient de l'extérieur ».

Aussi, pas plus la joie qu'éprouve Ella Maillart, que la dépression dont souffre Christina, ne proviennent du monde extérieur. Si Christina et Kini n'ont pas la même 
perception du monde qui les entoure, c'est parce qu'elles y projettent des humeurs différentes. Ella Maillart comprend que «beauté, tristesse, joie ne sont pas partie intégrante d'un objet, d'un évènement : elles n'existent nulle part ailleurs qu'en moi » (p. 125). Et ce qui vaut pour des sentiments, tels que la «tristesse » et la «joie», vaut aussi pour des sensations, «car le goût est en nous-mêmes et non dans ce que nous mangeons »(p. 249). Même le chaos européen, d'ailleurs, auquel Ella Maillart s'était montrée si sensible, n'est peut-être en fait que le produit d'un désordre intérieur tant « le chaos qui nous entoure est lié au chaos qui est en nous » (p. 26).

Aussi, même si Ella Maillart échoue à guérir Christina par le voyage, cet échec n'est pas inutile, puisqu'il lui permet de prendre pleinement conscience du caractère primordial de la vie intérieure. Non seulement, cette dernière « colore et conditionne la vie extérieure » mais elle « est plus proche de nous et d'une réalité plus essentielle» (MAILLART, 2001b : 221). Et l'on peut voir dans La Voie cruelle un parcours qui, au lieu de convertir Christina aux vues d'Ella Maillart, amène au contraire cette dernière à adopter la position de sa compagne : les humeurs du voyageur ne sont pas une projection du monde ; mais c'est le monde qui est une projection de ses humeurs.

Sans doute, fallait-il un voyage à deux pour qu'Ella Maillart aboutisse à cette conclusion. En effet, en voyageant seul, on peut toujours penser que les sentiments qu'on éprouve proviennent du monde environnant, et que toute personne, au même endroit, au même moment, éprouverait la même tristesse ou la même joie ; cependant, quand deux voyageurs qui suivent, en même temps, le même itinéraire, ne perçoivent pas le monde alentour de la même manière, alors force est d'admettre que la vie intérieure de chacun colore le monde extérieur différemment, et que le dedans prévaut sur le dehors.

C'est d'ailleurs en voyageant pour la première fois en tandem, en 1935, avec Peter Fleming, qu'Ella Maillart pressent le caractère irréductiblement personnel du voyage. En effet, quoique Peter Fleming et Ella Maillart aient suivi, en même temps, sans jamais se séparer, la même route, les récits que chacun écrit à son retour - Peter Fleming publie Courrier de Tartarie en 1936, et Ella Maillart, Oasis interdites, en 1937 - sont cependant très différents.

Le récit de Maillart complète Courrier de Tartarie, et donne la possibilité au lecteur des deux récits de considérer la façon dont les deux voyageurs - l'un masculin, l'autre féminin; l'un britannique, l'autre suisse; l'un pressé, 
l'autre privilégiant la lenteur - traduisent chacun à sa façon leur expérience sur le terrain en texte littéraire. (FORSDICK, $2008: 85$ )

En effet, bien qu'ils aient suivi ensemble le même itinéraire, les différences sont telles entre Peter Fleming et Ella Maillart, qu'ils n'ont pas vécu le même voyage. Aussi Ella Maillart comprend-elle, après la publication des deux textes, qu'un récit de voyage rend moins compte des empreintes que le monde peut laisser en nous - sinon, un même endroit, au même instant, laisserait la même trace - que des impressions qu'on a projetées sur lui.

Lors de ma traversée d'Asie avec mon camarade Peter Fleming, pendant des mois nous n'avons pu parler qu'ensemble. Bien que nous ayons suivi le même itinéraire, mon voyage diffère complètement du sien. La pensée individuelle colore le voyage comme si chacun portait des lunettes teintées différemment. En fait c'est notre pensée que nous projetons au dehors pour la déchiffrer ensuite lorsque nous rencontrons le prétendu monde objectif. (BOUVIER, MAILLART, SCHWARZENBACH, $2003: 189-190$ )

Or, c'est bien ce caractère subjectif du voyage, cette projection de la pensée sur le «prétendu monde objectif », pressentie en comparant Courrier de Tartarie et Oasis interdite, que le voyage aux côtés d'Annemarie Schwarzenbach lui permet d'établir de façon définitive dans La Voie cruelle.

\subsection{Les dialogues}

Ce n'est pas la première fois qu'Ella Maillart voyage à deux. Cependant, même si, comme le note Ella Maillart, «pendant des mois», Peter Fleming et elle n'ont pu «parler qu'ensemble », les dialogues occupent moins de place dans Oasis interdites que dans La Voie cruelle. C'est que, dans le premier, jamais la présence du voyageur Anglais à ses côtés n'oblige Ella Maillart à s'extraire du monde extérieur. Aussi, Oasis interdites présente-t-il les caractéristiques d'un récit de voyage traditionnel, où la narration des péripéties du voyage alterne avec la description de ce qui est vu - où l'aventure alterne avec l'inventaire, «ces deux composantes fondamentales de tout récit d'itinéraire »(LESTRIGANT, 1999 : 73).

Par contre, dans La Voie cruelle, la présence de Christina oblige sans cesse Kini à revenir du spectacle extérieur à l'habitacle de la voiture, des paysages à sa compagne - de la narration au dialogue : «Je m'efforçais de composer des articles, ou bien je me tourmentais au sujet de mon amie - proie de cette alternative qui, pendant tout le voyage, me faisait aller 
et venir du monde objectif au monde subjectif » (p. 137). Interrompue dans son observation du monde extérieur, la voyageuse l'est aussi dans l'écriture de son voyage. Aussi, l'irruption aux côtés des passages narratifs et descriptifs, qui forment la trame de tout récit de voyage, de longs passages dialogués, témoigne-t-elle de ce va-et-vient entre «monde objectif» et «monde subjectif», et traduit-elle textuellement ces interférences entre itinéraire (géographique) et interrogation (intérieure).

Ces dialogues entre Kini et Christina, qu'Ella Maillart n'a pas enregistrés et qu'elle écrit « dix ans plus tard» (p. 74), il se peut qu'ils soient en partie retranscrits à partir des notes voyage, en partie réécrits d'après mémoire. Il faudrait, pour cela, avoir accès aux carnets de routes de l'auteur.

Néanmoins, un passage du récit permet de supposer qu'Ella Maillart, plutôt que de retranscrire des conversations qu'elle aurait vraiment eues avec Annemarie Schwarzenbach pendant leur voyage, aurait plutôt entrepris dans La Voie cruelle de poursuivre avec son amie disparue un dialogue post mortem. En effet, d'une part, c'est le décès d'Annemarie Schwarzenbach, «morte paisiblement » après un accident de bicyclette (p. 312), qui incite Ella Maillart à écrire le récit de leur aventure. D'autre part, même après sa mort, Ella Maillart continue de s'adresser directement à son amie : «Aujourd'hui - dix ans plus tard - tandis que j'écris ces lignes, je pense que si le but de la naissance est de vivre longtemps, alors je gagne, Christina, puisque vous n'êtes plus parmi nous !» (p. 74). Ce discours, destiné à un « vous » qui n'est plus «parmi nous », et qu'Ella Maillart reprend dans l'«ENVOI » qui clôt le récit (p. 313), l'auteur l'adresse à la Christina qui n'est plus - autrement dit, non pas à la vraie Christina, mais à la Christina qu'elle porte en elle.

Or, on peut supposer que non seulement ce passage, mais tous les dialogues du récit sont, en quelque sorte, moins le souvenir d'une conversation passée, que la reprise, au-delà de la mort, d'une conversation interrompue. Comme si le moment d'énonciation des dialogues ne renvoyait pas au moment du voyage, mais bien au moment de l'écriture : autrement dit, comme si les dialogues entre Kini et Christina étaient prononcés dans l'instant de leur écriture. N'est-ce pas d'ailleurs, ce qu'Ella Maillart suggère en écrivant, à la fin du récit, que sa compagne de voyage était «présente» dans chacune de ses pages (p. 313) ? Aussi, les conversations qu'Ella Maillart développe au long du récit avec une interlocutrice moins externe qu'interne - avec une voix intérieure - relèvent-elles moins du dialogue entre deux personnes, que d'un dialogue entre deux facettes d'une même personne. 
Cette impression se trouve renforcée par l'usage d'un pseudonyme que des révélations sur la vie privée d'Annemarie Schwarzenbach - son divorce, sa dépression, sa morphinomanie, son homosexualité - ont rendu nécessaires, mais qui en même temps isole Christina de son référent, la coupe de sa réalité : le pseudonyme fait, en quelque sorte, d'une personne réelle, un personnage fictif. Contrairement au Peter Fleming d'Oasis interdites, la Christina de La Voie cruelle, plus malléable qu'un être de chair et d'os, se prête davantage au travail de l'imagination. Elle est moins un souvenir qu'une invention, moins un être réel qu'une projection de l'auteur - moins un avatar d'Annemarie Schwarzenbach qu'un double d'Ella Maillart. En effet, alors que Peter Fleming était trop différent d'Ella Maillart - par la nationalité, le sexe, la façon de voyager - pour en être le double, Christina, passée la première différence déjà relevée dans les rapports entre le dehors et le dedans - différence qui, d'ailleurs, s'abolit au cours du récit, Ella Maillart finissant elle aussi par accorder la primauté au monde subjectif - se révèle en fait semblable à sa compagne de voyage, et pour des raisons qui ne tiennent pas qu'à leur sexe ou à leur origine helvétique. Ainsi Christina permet-elle à Kini de découvrir une face ignorée de sa propre personnalité, comme dans ce cauchemar qu'elle fait lors du séjour à Istanbul.

Christina était sur le sol, repliée sur elle-même, exsangue, évoquant un chien mourant plutôt qu'un être humain. Debout près d'elle, je lui donnais des coups de pied tout en criant furieusement :

- Parlerez-vous, maintenant? Pourquoi ne me répondez-vous pas?

Je savais que si je parvenais à lui faire suffisamment mal, elle parlerait enfin, et alors tout serait arrangé. Essayant de lui causer le plus de douleur possible, je m'acharnais sur sa tête sans vie. Sans résultat. Mais il fallait qu'elle parle ! Enragée, je décochai un tel coup dans la masse souple de son ventre que je me tordis la cheville... et me réveillai ! Je tremblai encore d'une passion qui avait possédé tout mon corps de rêve. (p. 66)

D'une part, ce cauchemar révèle à Kini des aspects de sa propre personne qu'elle ignorait : «Je suis bouleversée de voir que j'ai en moi de telles possibilités de cruauté » (p. 67) ; mais d'autre part, il montre aussi qu'en essayant de contraindre Christina à parler, elle trouve un intérêt personnel aux révélations que peut lui faire son amie : «Ce cauchemar semble indiquer qu'il y a entre nous des correspondances plus profondes que nous pouvions le supposer» (p. 67). Car, malgré les différences de caractères, l'une et l'autre ont des préoccupations communes ; or, comme le montre ce cauchemar, il semble bien que Christina dispose de réponses que Kini veuille connaître à tout prix, la violence du rêve en révélant l'importance. Comme l'écrit Ella Maillart, 
[...] nous étions semblables dans notre dévotion envers quelque chose que nous ne pouvions pas nommer. Toutes deux nous avions voulu rester libres afin de mieux remplir en temps voulu une obligation suprême envers nousmêmes, épreuve et obligation dont la nature nous était encore cachée. (p. 130)

Par delà leur itinéraire vers l'Afghanistan, Kini et Christina poursuivent un but commun, dont elles sentent le caractère impérieux d' «obligation », mais dont elles ignorent cependant le nom et la «nature », comme une «dévotion » qui ne saurait encore à quel dieu elle est adressée. Or, ce but se trouve en elles-mêmes, et c'est moins par le voyage que par le dialogue qu'elles peuvent espérer atteindre une réponse commune. Aussi, Christina joue-t-elle dans le récit le rôle d'un double qui permet à Ella Maillart d'entretenir un dialogue avec ellemême et, par un jeu de questions-réponses, de chercher des solutions à ses propres interrogations.

Ainsi, non seulement, d'Oasis interdite à La Voie cruelle, la part du dialogue augmente, montrant l'importance que gagne l'aspect réflexif du voyage sur ses aspects narratif et descriptif, mais la nature du dialogue aussi se transforme. Les passages dialogués dans La Voie cruelle sont moins la reproduction de conversations authentiques entre Ella Maillart et Annemarie Schwarzenbach, que la poursuite d'un dialogue post mortem entre Kini et son double : le dialogue relève ici d'un procédé littéraire ; c'est la forme commode que l'auteur choisit de donner à une réflexion personnelle, qui tient moins, finalement, du dialogue que d'un monologue intérieur à deux voix.

\subsection{Les descriptions}

Le caractère introspectif du voyage amène Ella Maillart à introduire de longs dialogues - ou monologues - dans le récit de voyage. Cependant, même la description qui, avec la narration, est un des éléments constitutifs du genre, témoigne d'un va-et-vient entre le monde objectif et le monde subjectif. En effet, le récit d'Ella Maillart propose deux types de descriptions, dont l'une relève de la perception du monde extérieur, et l'autre manifeste au contraire la projection d'un monde intérieur - la description oscillant ainsi entre l'observation (photographique) et l'impression (picturale). 
Observation. Depuis son voyage dans le Kirghizistan, en 1932, Ella Maillart a fait de la photographie son «devoir de voyageuse » (MAILLART, 2001b: 96). À la manière d'un carnet de route, l'appareil photo permet d'enregistrer le voyage ; les prises de vues sont en quelque sorte des prises de notes visuelles qui, au retour, serviront de support à l'écriture du récit. Ainsi, dans La Voie cruelle, comme dans Oasis interdites, «le texte adopte même des stratégies photographiques, esquissant des scènes ou des personnages dont l'image se trouvait sans doute devant l'écrivain pendant qu'elle rédigeait son manuscrit » (FORSDICK, 2008 : 40). Ce sont ces images qui permettent, par exemple, à Ella Maillart de décrire avec précisions les nomades rencontrés :

Les femmes dans leur costume rouge et noir avaient des traits romains encadrés de petites tresses serrées et austères, comme une chaîne métallique relevée pour laisser le front découvert. Les fillettes, une pièce d'argent fixée au bout d'une mèche courte, portaient de longues robes ornées d'amulettes... (p. 275)

La richesse des détails - les costumes, les coiffures, les ornements - témoigne ici d'une précision tout aussi ethnographique que photographique.

Cependant, les images photographiques ne sont pas seulement destinées à devenir des images écrites : elles peuvent aussi intégrer le récit sous forme d'illustrations. «Bien que les actuelles éditions de poche des ouvrages de Maillart soient dépourvues d'images, les originales se vendaient en partie grâce à leur riche iconographie » (FORSDICK, $2008: 39$ ). L'édition originale de La Voie cruelle ne comptait pas moins de trente-trois photographies prises par l'auteur. Or, l'image photographique mise en regard de sa transcription n'a pas pour seule fonction de confirmer les informations déjà contenues dans le texte. En effet, si la photographie permet, d'une part, d'illustrer le récit, elle permet aussi d'en attester l'authenticité : elle ajoute au voyage relaté la dimension du vécu. Elle atteste non seulement que les paysages, les monuments et les gens sont bien tels que l'auteur les a décrits, mais aussi que c'est bien lui qui les a vus. La photographie sert au voyageur de certificat d'autopsie. Elle remplit une fonction qui, dans les récits de l'époque classique, est dévolue aux «formules de véridiction» (PERRONE-MOISES, 1997 : 86) par lesquelles le voyageur atteste la réalité de son voyage en assurant régulièrement avoir vu ce qu'il évoque par luimême. Aussi, la photographie épargne-t-elle au voyageur moderne le déploiement de toute une rhétorique autrefois destinée à convaincre les lecteurs de sa bonne foi. 
Toutefois, qu'elles soient sous leurs formes écrites ou visuelles, les images photographiques visent à reproduire fidèlement ce qui a été vu, à rendre compte du monde extérieur.

Impression. Cependant, Ella Maillart reconnaît aussi les limites de la photographie - et pas seulement de la photographie en noir et blanc. En effet, à la mosquée de Mechhed, dont les minarets sont d'une « splendeur inégalée », tandis qu'elle cherche «l'angle d'où un cliché en couleurs rendrait justice à ces joyeux contrastes », force lui est d'admettre que « la photo ne peut pas plus reproduire les tons veloutés de ces émaux qu'elle ne peut capter le lustre d'un riche tapis » (p. 195). Et c'est alors au texte de jouer un rôle non pas redondant mais complémentaire, et de suppléer aux insuffisances de l'image photographique, en ajoutant « la densité », «l'éclat», «les contrastes » ou encore les «tons », que cette dernière ne peut capter.

Les exemples sont innombrables qui témoignent du soucis d'Ella Maillart de rendre dans le texte toutes les nuances des couleurs : «pas un arbre en vue, rien qu'un monde de terre jaune où tout était jaune, y compris les murs en pisé, mais où la blancheur des turbans et le rouge amarante des caftans vibraient joyeusement comme dans un tableau de maître » (p. 219) ; « je vis une scène que j'aurais voulu peindre : intense bleu du ciel, paille dorée empilée sur les toits, brun des murs en pisé, robes rouges des femmes tissant à même le sol » (p. 283). Comme le reconnaît Ella Maillart, devant ces paysages dont les couleurs semblent imiter « un tableau de maître », la description exige moins une précision de photographe qu'une sensibilité de peintre. Elle relève moins de l'image photographique que de l'image picturale.

Or, pour Ella Maillart, les qualités picturales d'un paysage ou d'une personne dépendent moins de l'objet regardé que du sujet qui observe. En effet, d'une part, la perception des couleurs dépend de la capacité des organes sensoriels à les percevoir. Un animal peut fort bien ne pas percevoir les mêmes couleurs qu'un être humain ; mais aussi, la perception des couleurs peut varier d'un homme à l'autre. Il est alors essentiel de connaître les caractéristiques de ces organes pour ne pas attribuer des particularités qui ne tiennent qu'à eux à la réalité qu'on observe : «Si je ne sais pas que mes lunettes sont vertes, comment pourraisje imaginer que la route est plus verte pour moi que pour les autres ?» (MAILLART, 2001b : 222)

D'autre part, entre une photographie - du moins la photographie de voyage telle que la pratique Ella Maillart - et un tableau, la différence ne tient pas seulement aux qualités des couleurs. De la perception des caractéristiques d'un paysage - ou d'une personne - à 
l'appréciation de leur beauté, l'image quitte une visée strictement documentaire pour adopter une visée artistique, - l'ethnographie pour l'esthétique. Or, la beauté d'un lieu, d'un visage, comme aussi la beauté d'une musique, dépend moins des qualités de l'objet que de la sensibilité du sujet: «la beauté d'un mur, la tristesse d'une symphonie ne peuvent probablement pas être ressenties par mon garçon laitier » (p. 125). Il ne s'agit plus, ici, des seules propriétés de l'organe sensoriel, mais du niveau d'éducation du voyageur, dont les sens, selon le degré d'éducation, auront pu rester grossiers ou au contraire s'affiner.

Enfin - et surtout - la beauté du monde dépend de l'état d'esprit de celui qui regarde : « Notre état d'âme toujours changeant conditionne, transforme même, les paysages et les gens que nous rencontrons » (p. 42). Aussi, la beauté picturale qu'Ella Maillart croit découvrir dans certains paysages n'est en fait que la projection d'un état d'âme sur le monde ; et ces «joyeux contrastes », cette «note gaie» (p. 195), ce blanc et ce rouge qui «vibraient joyeusement» (p. 219), toute cette jubilation des couleurs n'est en fait que la projection sur le monde extérieur d'une gaieté toute intérieure.

Les premières heures de la journée me donnèrent de la joie. Les couleurs étaient vives mais fondues, comme bordées de nacre - l'émeraude de l'herbe courte, l'orange et le violet des falaises, le jaune des champs, les brillantes feuilles des peupliers. Et là-haut régnait le bleu du ciel, un bleu riche et profond, semblable à celui qui domine les champs de neige en Suisse. (p. 264)

Si l'on en croit Ella Maillart, entre la première phrase de ce passage et les suivantes, la relation sous-entendues par l'asyndète n'est pas, ici, d'un effet (une émotion au réveil) vers ses causes (les couleurs du paysage), mais, au contraire, d'une cause vers ses effets : c'est bien la «joie » qu'éprouve Ella Maillart au lever du jour qui orne le monde de couleurs joyeuses. Au contraire si Christina peut demeurer indifférente au «"cri" d'une fleur rouge contre le bleu du ciel» (p. 128), ce n'est pas faute de percevoir les couleurs, mais parce qu'elle n'en projette aucune sur le monde.

Le lac du Band-i-Amir, qui repose au cœur d'une vallée afghane, et dont la surface tantôt reflète le monde avec la précision d'une image photographique, et tantôt ajoute au monde d'autres reflets comme des touches de couleurs sur une toile, résume assez bien les différences entre observation et impression.

Tandis que le lac devenait une masse frémissante de métal en fusion, le soleil couchant couvrait les falaises de couleurs amarantes, lilas, et jacinthe. Plus tard, au moment d'aller nous coucher, il y eut deux voies lactées, celle 
qui était à nos pieds paraissant aussi mystérieuse que celle qui nous dominait.

Mais c'est l'aube sans vent qui nous offrit le plus beau spectacle. [...] Nous vîmes à nos pieds une vaste surface de bronze poli, gigantesque miroir arrondi qui reproduisait chaque teinte, chaque déclivité, chaque ravin et chaque variation du monde supérieur.

Ce miroir enchanté n'avait pas plutôt ouvert nos yeux à la beauté de la terre qu'il disparut ; une brise vivante le transforma en une arène de lapis-lazuli suspendue à mi-chemin entre de basses terres pâles et de hautes falaises rougissantes. (p. 279)

Bien que ce passage décrive un paysage lacustre, il n'est pas placé sous le signe de l'eau mais plutôt sous celui de la terre : les feux du soleil couchant transforment en effet l'eau du lac en «masse frémissante de métal en fusion » qui, bientôt refroidie, se durcit en «vaste surface de bronze poli ». Sous l'action alchimique du feu, l'eau s'est ainsi transformée en un «gigantesque miroir arrondi ». S'y reflètent alors aussi bien la voie lactée, de nuit, que «chaque teinte, chaque déclivité, chaque ravin », à l'aube. Et le miroir reproduit si bien «chaque variation du monde supérieur » que non seulement la voie lactée réfléchie paraît «aussi mystérieuse » que l'autre, mais que le reflet semble même révéler « la beauté de la terre » mieux que le paysage original. Cependant, une «brise » trouble la perfection du reflet, la faisant même disparaître ; et, sous l'action de l'air, et non plus du feu, l'eau connaît alors une seconde métamorphose : la surface polie du bronze se fragmente en milliers de «lapislazuli ». Le métal se transforme en pierres précieuses. Cependant, si l'on reste toujours dans le règne des matières terrestres, cette nouvelle transformation apporte un changement important : alors que le lac, sous forme de miroir, reproduisait paisiblement les couleurs du monde, fragmenté en milliers de lapis-lazuli, il ajoute ses propres éclats bleutés à la blancheur des « basses terre » et la rougeur des « falaises ». Or le lac est un peu à l'image du voyageur : en paix, il reproduit comme un miroir - ou comme l'image photographique - le monde alentour dans ses moindres détails ; troublé par une émotion - la joie, la tristesse - il y projette au contraire comme un pinceau sur la toile ses propres couleurs.

En passant d'une description photographique à une description picturale, Ella Maillart passe ainsi de la reproduction du monde extérieur à la projection d'un monde intérieur - de l'observation à l'impression. Aussi, les deux types de descriptions renvoient donc aux deux dimensions - objective et subjective - du voyage, tout en montrant combien la seconde l'emporte sur la première : en effet, si la beauté du monde objectif dépend de dispositions subjectives - capacités sensorielles, niveau d'éducation, émotions - si la beauté extérieure est avant tout la projection d'une beauté intérieure, c'est donc au monde subjectif qu'il convient avant tout de s'intéresser. 
Il est clair, cependant, que l'on ne saurait confondre la description écrite avec une photographie ou une peinture, le pouvoir du langage étant bien différent du pouvoir de l'image photographique ou picturale. Et d'ailleurs, quand Ella Maillart évoque ses difficultés à rendre certaines couleurs, il faut bien admettre qu'elle y arrive tout de même, et avec le seul recours du langage. Il en va ainsi, par exemple, du bleu turquoise.

La turquoise des vieux monuments turcs ou persans est incomparable. Contre le ciel, elle paraît verte ; contre le feuillage de Samarkand, elle est bleue. Elle est à la pierre turquoise ordinaire ce que les plumes de martinpêcheur sont à l'azur du ciel. J'aimerais l'appeler « bleu d'Asie centrale » en souvenir d'un lac glaciaire des Tian Shan qui déployait cette radieuse saturation bleu ciel à reflets verts. (p. 132)

Aussi, ce que montre Ella Maillart, sans peut-être en saisir toute la portée, c'est que, même si l'écriture semble vouloir imiter la photographie ou la peinture, elle relève néanmoins d'un autre langage, doté de ses propres ressources. Et que si la pellicule ou le pinceau échouent à saisir ou produire «cette radieuse saturation bleu ciel à reflets verts », il est toujours possible avec des mots de créer un «bleu d'Asie centrale » d'un turquoise plus chatoyant que «la pierre turquoise ordinaire», de même que, si l'on en croit Fernando Pessoa, « os campos são mais verdes no dizer-se do que no seu verdor » (PESSOA, 1999 : 63).

Contemplation. Il semble, cependant, qu'on trouve dans La Voie cruelle un dernier type de description, correspondant à un troisième type de relation entre le dedans et le dehors : dans l'observation, le monde laisse une empreinte sur la rétine de l'observateur comme sur une émulsion photographique; dans l'impression, au contraire, l'observateur projette sur le monde ses humeurs, comme un peintre couvre sa toile de couleurs. Dans un cas, le monde vient à l'observateur, dans l'autre, celui-ci vient au monde.

Or, si l'on en croit Ella Maillart, il est des lieux où la distance entre le monde intérieur et le monde extérieur est parcourue d'une troisième manière : où il arrive que l'observateur, au lieu de se projeter sur le monde, se sente attiré par lui. C'est une propriété, semble-t-il, des lieux de cultes, cathédrales et mosquées. «Quand on a l'habitude d'être happé vers l'avant par la nef de nos cathédrales, c'est une curieuse expérience que d'entrer sous certains dômes, où l'on se sent comme aspiré vers le haut» (p. 64). Et c'est une attraction de ce type qu'Ella Maillart éprouve en entrant dans « le vieux tombeau d'Oldjaytu »: 
Une fois à l'intérieur de ce monument profané, son plan très simple devenant symbolique, nous étions immobiles, remplies de respect envers quelque chose de grand. Lentement mon esprit s'éleva vers la paix et la plénitude de l'intérieur du dôme qui nous dominait de quelque quarante-cinq mètres. Seules les formes comptaient, car peintures et inscriptions n'étaient plus que des ombres à peines visibles. La douce lumière qui pénétrait par les hautes baies était comme la promesse d'une riche et large contemplation - si jamais on parvenait à s'élever jusqu'à elles ! (p. 132)

Le lieu impose tout d'abord l'immobilité : mais ce n'est pas parce qu'il écrase sous sa monumentalité ; au contraire, c'est parce qu'il aspire vers « quelque chose de grand ». Ainsi, on trouve non seulement dans ce passage la même élévation qui caractérise le mouvement ascensionnel du voyage - bien qu'elle se produise ici non plus à l'échelle géographique, mais au niveau intime - mais aussi l'expérience d'une dilatation. Ella Maillart se sent non seulement aspirée hors d'elle-même, invitée par la voûte du dôme à franchir ses propres limites, mais aussi à s'élargir vers la «promesse d'une riche et large contemplation ». Dans ce lieu, il semble que toute couleur ait disparu, que «peintures et inscriptions » ne soient plus que « des ombres à peines visibles »; privé de couleur, ce lieu n'est plus que « formes », dont «le plan très simple » tend même à devenir «symbolique » : or, si les couleurs du paysage sont les reflets d'une impression, les formes, elles, invitent à la contemplation.

Cette sensation d'être aspiré hors de soi, comme si le dedans se dilatait aux dimensions du dehors, n'est pas réservée aux seuls lieux de culte. Un paysage peut s'y prêter, à condition que, comme le tombeau d'Oldjaytu, il soit moins composé de couleurs que de formes simples. «Lorsqu'un arbre ou un roc se dresse isolé dans le vide d'un désert, il participe à la grandeur environnante en centrant sur lui-même les radiations de l'immensité ; c'est là le charme du Tibet, ce vide autour du plus simple accident de terrain » (p. 131). Il est ainsi des paysages dont la simplicité aspire vers le seul accident de terrain notable, et prédispose ceux qui le contemplent à la méditation; et peut-être faut-il chercher dans la pauvreté du paysage tibétain la grande spiritualité de ses moines. C'est en tout cas un paysage de ce type, en Afghanistan, qui provoque en Ella Maillart une expérience contemplative.

Dans ce grand espace vide, il y avait à ce moment moi-même et la terre, et nous formions une paire d'amis s'entendant bien. Réduit à l'essentiel (un squelette de collines revêtues d'une chair maigre), le monde n'avait pas grand-chose à m'offrir, mais il me plaisait ainsi. Rien de trop, et presque rien du tout. Parfois, au milieu d'une succession d'épaules ocrées, un carré de luzerne verte dans une aisselle maigre; arêtes avec leur ligne fuyante qui s'estompe et vous attire; autant de pistes suivies par mes idées bondissantes, utilisant ces rudiments de paysages pour les associer à d'autres étapes parcourues autrefois près de l'Issyk Koul ou dans les Kunlun. Fascination 
d'un horizon qu'on voudrait atteindre mais que chaque pas repousse plus loin.

Être acceptée par la terre. Comprendre sa signification. Puis sentir combien elle est un tout, enfin libérée de l'aveuglement inhérent à un amour partiel. (p. 273)

L'espace, ici, offre peu de prises à la description : il s'agit moins d'un paysage que de «rudiments de paysage ». On dirait moins un paysage fini qu'une esquisse. Presque sans « chair », il est réduit à son « squelette »; presque sans matière, il se résume à quelques lignes et quelques touches de couleurs : des collines « ocrées », « un carré de luzerne verte ». Rien qui justifie qu'on en prenne une photographie, ni qu'on en fasse un tableau. Rien de notable ni de pittoresque. Pourtant, ce paysage non seulement plaît à Ella Maillart, mais il l'« attire », il exerce sur elle une «fascination»: il lui parle. À eux deux, ils forment « une paire d'amis s'entendant bien ». Alors, tandis que le paysage s'humanise, que ses courbes dessinent des «épaules », qu'un de ses plis forme une «aisselle», il semble inversement que l'humain s'élargisse aux dimensions du paysage, qu'il confonde ses limites avec celles d'un horizon «que chaque pas repousse plus loin»: tandis que l'espace se rapproche de l'humain, l'humain se dilate aux dimensions de l'espace. Le dehors et dedans se confondent; la conscience s'élargit aux dimensions de l'espace, où les « idées bondissantes » peuvent aller à leur gré. L'aspiration de la voyageuse par l'espace aboutit à leur union. C'est un moment contemplatif où, brusquement, Ella Maillart éprouve la sensation de ne faire qu'un avec le monde, où monde extérieur et monde intérieur ne s'imposent plus l'un à l'autre, mais se fondent l'un dans l'autre. Il s'agit d'une contemplation, comme celles dont parlent les mystiques chrétiens ou les moines zen : un moment de communion avec le monde où Ella Maillart, «acceptée par la terre », cesse d'en être une simple partie pour se fondre dans le « tout ».

Or, ce qui se libère dans ces instants de contemplation, ce qui est ainsi absorbé - on pourrait même dire gobé - par le monde, c'est un moi profond brusquement débarrassé du moi superficiel qui lui servait de coquille. Pour Ella Maillart, en effet, chaque personne est composée de deux moi bien distincts. Il y a d'une part, l'« ego » - ce qu'Ella Maillart appelle aussi le «moi fatigant» (p. 180) - et, d'autre part, le «Centre», «notre "note" la plus profonde »-qu'elle nomme aussi « notre personnalité » (p. 310).

Aussitôt dépassés notre angoisse, notre lâcheté, notre vanité, notre patience, notre courage ou notre amour pour un but limité ou pour un seul être, alors 
nous atteignons notre «note» la plus profonde, notre Centre, le même en chacun de nous, ce son silencieux auquel toutes les diversités se réfèrent, toutes les différences que nous croyions divergentes et isolantes se réduisent réellement. (p. 75)

L'ego est le moi superficiel, autrement dit le moi situé en superficie et le moins authentique ; c'est lui qui fait de chaque personne un individu distinct des autres ; c'est dans l'ego qu'on trouve «lâcheté », « vanité », « patience », « courage », « amour », toutes les particularités, «toutes les différences », qui font d'une personne un être singulier, défini par une combinaison de désirs, de tourments et d'humeurs. Or, cet ego, qui détermine le caractère unique de chaque personne, qui la différencie de toutes les autres, est aussi ce qui l'isole : ce qui distingue est aussi ce qui sépare. Par contre, le «Centre » est notre moi profond : à la fois le plus en profondeur et le plus authentique. Quelque soit le nom qu'elle lui donne, Ella Maillart lui attribue généralement le déterminant «notre », pour en montrer tout à la fois le caractère singulier et général, car ce moi contient la part commune à tous les êtres humains : si, en superficie, nous sommes tous différents, en profondeur, nous sommes tous identiques. S'il y a autant d'egos qu'il y a de personnes, il n'y a par contre en chaque personne qu'une seule «note fondamentale». Cette note, d'ailleurs, n'est pas seulement commune à tous les êtres humains : elle est la même pour tous les êtres vivants. L'ego est la marque distinctive de l'homme, ce qui le singularise non seulement parmi les siens, mais aussi parmi les êtres vivants ; la «note fondamentale » est au contraire la part commune à toutes les créatures, aux hommes, aux animaux et même aux plantes.

Le lis ou le reptile, le chat ou l'ortie savent être splendidement, totalement eux-mêmes. Créatures tourmentées par nos contradictions innées, nous devons dénouer l'écheveau qui est en nous, devenir assez simples pour pouvoir libérer la «note» fondamentale de notre Centre. (p. 74-75)

L'ego et le Centre articulent donc multiplicité et unicité de manière inverse : dans l'ego, une multitude de «contradictions innées » font de chaque homme un individu unique ; dans «notre Centre », au contraire, une «note» unique apparente la multitude infinie des êtres. Or, l'un enveloppe l'autre, l'enferme, l'étouffe. L'ego (superficiel) enveloppe le Centre (profond) comme une peau isolante. Aussi, doit-on se débarrasser de l'ego, afin de «libérer » la «note fondamentale $»$.

Ainsi, alors que l'industrie a rompu le lien qui unissait l'homme à son milieu, et qu'elle détruit les cultures qui vivaient harmonieusement avec la Nature, Ella Maillart cherche à rétablir un lien intime entre son être et le monde - parvenant même, dans certains moments 
contemplatifs, à reformer cette unité perdue. Là, sans doute, se trouve le remède. Pour renouer le pacte que, dans son aventure prométhéenne, l'Européen à rompu avec la Nature, il faudrait que chacun, à l'imitation d'Ella Maillart, atteigne le Centre qui se trouve en lui comme en chaque être vivant. Mais comment se défaire de l'ego ? Comment libérer notre note fondamentale ? La contemplation de paysages désolés, dont la simplicité fait écho à la simplicité du Centre, ramène Ella Maillart dans le sein de la Nature. Mais «les moments de plénitude totale, où la vie apparaît telle qu'elle pourrait être, sont trop rares » (p. 127-128). Comment rendre cette plénitude permanente? Comment abolir le «moi » pour mieux se fondre dans le tout?

Et qu'aurait dit Nicole si je lui avais avoué que mon vœu suprême était de me débarrasser de mon moi fatigant, de mes désirs toujours changeants et presque toujours dénués de sagesse ? Je ne voulais pas le faire en me tuant, ou en me jetant à corps perdu d'une contrée à l'autre comme je l'avais fait jusqu'ici [...]. Il doit y avoir un procédé moins sentimental qui atteigne le même but, un moyen rationnel de percer cet ego ou encore de le transmuer. (p. 180)

Comme Ella Maillart le reconnaît elle-même, si le suicide n'est pas une solution, le voyage non plus ne saurait venir à bout de son «moi fatigant». Car si, tant qu'elle cherchait un Éden géographique, se «jeter d'une contrée à l'autre» pouvait avoir un sens, cela ne saurait être d'un grand secours quand il s'agit d'atteindre le Centre en chacun de nous. C'est en Inde, en suivant les enseignements de deux maîtres spirituels, qu'Ella Maillart trouvera finalement le «moyen rationnel» permettant de «percer» ou de «transmuer» l'ego. Elle fera le récit de cette expérience dans Ti-Puss. Dans La Voie cruelle, elle laisse simplement entendre que les voyages ne sauraient lui permettre d'atteindre son but; aussi paradoxal que cela puisse paraître, elle écrit un récit de voyage sur l'inutilité de voyager. Cependant, elle suggère aussi qu'il est une «voie », celle qui donne son titre au récit, la «voie cruelle » qu'emprunte Christina, une voie qui n'est pas destinée aux voyageurs, mais réservée aux écrivains. Et si Ella Maillart passe de l'espace extérieur à l'espace intérieur, son amie lui montre comment passer de ce dernier à l'espace du langage. 


\subsection{La voyageuse et l'écrivain}

La primauté du monde subjectif sur le monde objectif, qui conduit Ella Maillart à laisser l'exploration pour l'introspection, se traduit donc, textuellement, par la place accordée au dialogue et par des descriptions qui relèvent davantage de l'impression - ou de la contemplation - que de l'observation. Cependant, Ella Maillart ne se contente pas d'apporter quelques ajouts ou retouches à un genre qu'elle a déjà plusieurs fois pratiqué : elle en remet aussi en question l'utilité. Si le voyage ne saurait conduire au Centre, à quoi bon continuer de voyager et d'écrire des récits de voyage ? Une autre voie, une autre écriture, ne se prêteraientelles pas davantage à l'exploration du monde intérieur ?

Pour Ella Maillart, le voyage - l'expérience de l'Ailleurs, la rencontre de l'Autre - a précédé l'écriture. Avant son premier départ, en effet, elle n'avait jamais eu l'idée d'écrire. Et même après, Ella Maillart aurait pu continuer de se passer d'écrire ; pour elle, en effet, contrairement à Nicolas Bouvier, le voyage n'a pas imposé l'écriture comme une nécessité. $\mathrm{Si}$, malgré tout, elle s'est mise à écrire, c'est que le récit d'un premier voyage lui a fourni l'argent nécessaire au financement d'un deuxième. En effet, en 1930, les droits versés pour son premier livre, Parmi la jeunesse russe. De Moscou au Caucase (1932), lui permirent de repartir en Russie.

Le jour où le livre sortit, la maison Fasquelle me versa six mille francs ; c'était plus d'argent que je n'en avait jamais eu, et vu le prix très bas des voyages en troisième classe en Russie, c'était assez pour me transporter bien loin à l'est de Moscou. (MAILLART, 2001b : 74)

Cette fonction économique de l'écrit, Ella Maillart la rappelle plusieurs fois dans $L a$ Voie cruelle, qu'il s'agisse de «l'argent » versé, avant le départ, pour son «dernier livre » (c'est-à-dire Oasis interdites), de l' «avance sur l'histoire de [sa] randonnée afghane » (p. 26), qui devrait permettre de subvenir aux frais du voyage, ou encore, à la fin du livre, quand elle s'apprête à écrire le récit de ses aventures maritimes de jeunesse : «Afin de me procurer de quoi vivre, je commençai à écrire Gypsy Afloat avec l'espoir que suffisamment de gens l'achèteraient » (p. 305). Aux livres, il faut aussi ajouter les articles écrits par Kini et Christina pour des revues, comme le Geographical Magazine (p. 74), dont le caractère alimentaire est d'autant plus évident que, comme le rappelle Ella Maillart, «notre paie dépendait non de la longueur de la route mais de la surface de papier que nous noircirions !» (p. 86). Dans le 
rapport économique que l'écriture et le voyage entretiennent, c'est bien la « longueur de la route » qui dépend de «la surface de papier» noircie (Ella Maillart ne dit pas écrite), et non pas l'inverse.

Aussi, dès ses premiers essais d'écriture, Ella Maillart n'a-t-elle jamais prétendu au statut d'écrivain. Au retour de son premier voyage en Russie, un article écrit en anglais sur le Haut-Caucase est refusé par une agence américaine : "Ma déception ne fut pas grande », avoue Ella Maillart, « car je ne m’étais jamais crue écrivain » (MAILLART, 2001b : 71). «Je ne suis pas écrivain » (Id. : 74) est d'ailleurs la réponse qu'elle donne, quelques temps après, à l'éditeur Charles Fasquelle qui lui demandait d'écrire sur ce même voyage. Plus tard, bien qu'elle ait publié trois récits de voyage, dont le dernier - Oasis interdites - lui a même apporté une renommée internationale, c'est encore la même dénégation que l'on retrouve dans une lettre adressée le 2 mars 1941 à sa mère depuis l'Inde où elle a trouvé refuge : «tu sais que je ne suis pas écrivain dans l'âme ; et avant de continuer à écrire des livres imparfaits autant qu'inutiles, cela vaut la peine de réfléchir» (MAILLART, $2003: 152$ ).

Quand dans Croisières et caravanes, Ella Maillart revient sur l'écriture des trois récits publiés entre 1931 et 1936, c'est en général pour souligner les difficultés de cette activité. Avec Parmi la jeunesse russe, elle découvre tout d'abord le caractère laborieux de l'écriture : «que de pages furent maintes fois recommencées, et que de soupirs elles m'arrachèrent !» (MAILLART, 2001b: 74). Avec Oasis interdites, son troisième récit, non seulement l'écriture ne cesse d'être un « effort épuisant», mais elle en éprouve aussi plus fortement les limites : «On croit que c'est amusant d'écrire un livre ; je ne suis pas du tout de cet avis, on se rend trop bien compte que l'on n'arrive pas à exprimer les choses les plus importantes et qui demeurent insaisissables »(Id. : 208). Avec Des Monts célestes au sables rouges, qui s'inscrit entre les deux autres livres, les peines de l'écriture effacent même les «joies » du voyage :

Les joies de cette randonnée, qui avaient été brèves mais intenses, furent chèrement payées par les cinq mois d'été que je passai rivée à ma table à écrire: les conseils d'un écrivain expérimenté me manquaient considérablement, et j'avais le sentiment attristant de gaspiller un été qu'il eût mieux valu passer en plein air. (Id. : 155)

Ella Maillart met ici le doigt sur l'une des contradictions entre l'écriture et le voyage qui, pour le voyageur, rend d'autant plus pénible les travaux d'écriture. Car si le voyage est une activité nomade, l'écriture est au contraire une activité sédentaire ; si l'un est associé au mouvement et au «plein air », l'autre est « rivée » à l'immobilité et à l'enfermement. Par 
cette «alternance entre le voyage et l'écriture qui sera celle de son existence au cours des années trente », Ella Maillart fait ainsi la dure expérience du «rythme de vie d'un écrivainvoyageur, dont les périples se parsèment nécessairement de périodes sédentaires pendant lesquelles les mémoires du voyage se transforment en texte » (FORSDICK, $2008:$ 10). Or si certains écrivains-voyageurs trouvent un équilibre dans cette alternance sans laquelle le voyage ne saurait se transformer en récit, Ella Maillart ne parviendra jamais à faire vraiment de cette contradiction entre deux activités si opposées une nécessaire complémentarité. Pour elle, le voyage pourrait fort bien se satisfaire à lui-même.

Aussi les difficultés qu'elle éprouve à écrire l'amènent-elles à chercher ailleurs le financement de ses voyages. Et, au début de La Voie cruelle, elle attend de son séjour en Afghanistan, dont l'un des objectifs est d'étudier les tribus nomades de l'Asie centrale, qu'il lui permette enfin d'abandonner l'écriture :

Lorsque j'aurai collectionné des faits nouveaux concernant ces tribus, je serai enfin admise dans la confrérie des ethnographes. Alors tout sera parfait: j'appartiendrai à une organisation, ce sera mon métier de vagabonder, et je n'aurai plus la tentation d'écrire des livres pour vivre. (p. 23)

C'est bien «vagabonder», et non pas «écrire » qui, aux yeux d'Ella Maillart, constitue son vrai «métier». Ella Maillart aurait pu voyager sans écrire, si elle n'avait trouvé dans l'écriture sa principale source de revenu. Elle ne voyage pas pour écrire, mais écrit pour voyager. Le voyage est la véritable fin ; l'écriture, n'est qu'un moyen.

Dans La Voie cruelle, d'ailleurs, les deux figures de Kini et de Christina permettent à Ella Maillart de bien poser la différence entre la voyageuse et l'écrivain. Pour Ella Maillart, cette différence se perçoit à deux niveaux : la qualité du texte et la finalité de l'écriture.

Les deux amies, au long de la route, écrivent des «lettres de voyage promises à une agence de presse » $\left(\right.$ p. 73) ${ }^{16}$. Ella Maillart profite de cette tâche commune pour comparer leurs pratiques de l'écriture. Toutes deux écrivent «lentement»; toutes deux sont «mécontentes » de leurs productions. Cependant, cette difficulté commune recouvre, pour Ella Maillart, de profondes différences. D'une part, bien que cela ne change en rien son rendement, Christina

\footnotetext{
${ }^{16}$ Les lettres écrites par Annemarie Schwarzenbach ont été réunies dans un recueil intitulé Où est la terre des promesses? (Payot, 2000)
} 
bénéficie d'un «entraînement supérieur »; d'autre part, au vu du résultat, l'écriture de Christina est «plus coulante». Aussi, pour Ella Maillart, la différence de qualité entre ses textes et ceux de Christina ne fait aucun doute, bien que cette dernière essaie de la convaincre du contraire :

- Mais, Kini, vous vous trompez. Mes livres sont loin d'avoir le même tirage que les vôtres. Et vous savez que je n'ai pas encore été traduite !

- Votre remarque est aussi enfantine que votre désir de célébrité. Les informations de Reuter sont traduites dans toutes les langues, elles ont davantage de lecteurs que le meilleur poème du siècle. En ce moment vous écrivez des articles sans valeur afin de subvenir fièrement à vos besoins ; laissez cela à ceux qui doivent le faire ! (p. 73)

Aux critères quantitatifs que présente Christina - le tirage et le nombre de traductions - Kini oppose aussitôt un critère qualitatif : la différence entre des «articles sans valeur » et « le meilleur des poèmes », entre « un médiocre "papier" » (p. 73) et de «bonnes pages » (p. 74). Autrement dit, les textes d'Ella Maillart relèvent du journalisme, et ceux de Christina, de la littérature.

Aussi, bien que les deux rencontrent des difficultés pour écrire, ces difficultés ne sont pas du même ordre. Ella Maillart souffre parce qu'elle se sent peu d'affinités pour l'écriture ; Christina, parce qu'elle porte l'écriture à un degré élevé d'exigence. L'une peine à trouver les mots exacts, ceux qui disent précisément son voyage ; l'autre, à trouver les mots justes, ceux qui sonnent d'une façon satisfaisante. Et le travail qu'opère Christina sur les mots est bien différent :

Les mains étaient celles d'un artisan patient qui sait ciseler une ligne pure : je l'ai vue placer successivement sept feuilles blanches dans la machine à écrire avant qu'un paragraphe ait atteint le dessin aisé et parfait qui seul pouvait la satisfaire. (p. 25)

Cependant, quelles que soient les difficultés rencontrées par Christina, Ella Maillart ne doute pas que «le moment viendra où [Christina sera] si fortement inspirée [qu'elle sera] comme emportée par [son] travail » (p. 262). Ce qui se produit à la fin du récit lorsque, «inondée par le courant d'une riche inspiration dont elle s'émerveillait, elle commença un livre, Das Wunder des Baumes (« Le Miracle de l'arbre »)» (p. 310).

Par ailleurs, à cette différence de qualité, s'ajoute une différence de finalité. Pour Ella Maillart, on l'a vu, l'écriture de ses récits lui permet de financer ses voyages. Par contre, pour Christina, l'écriture ne saurait être un moyen. «Écrire était le seul rite de sa vie : elle y 
subordonnait tout » (p. 25). Il est vrai qu'elle s'intéresse à l'archéologie, et si Ella Maillart se rend en Afghanistan pour étudier des tribus nomades, Christina s'y rend pour participer à des fouilles. Cependant, l'intérêt commun qu'éprouve les deux amies pour les sciences humaines recouvre lui aussi une différence essentielle : si Ella Maillart rêve de devenir ethnologue pour cesser d'écrire, "Christina savait qu'elle ne deviendrait pas une archéologue. Sa seule ambition était d'écrire »(p. 261). Pour Christina, aucune activité ne saurait se substituer à l'écriture : «Christina ne vivait que pour écrire » (p. 39) ; et même si les résultats ne sont pas toujours à la hauteur de ses attentes, elle n'en demeure pas moins « un écrivain-né » (p. 262). Si Kini écrit pour vivre, Christina, elle, vit pour écrire.

Ainsi le récit place-t-il très clairement côte à côte une voyageuse et un écrivain pour lesquelles l'écriture ne représente pas du tout le même enjeu. Pour Ella Maillart, l'écriture est un moyen parmi d'autres - un moyen à défaut d'autres - lui permettant de poursuivre une vie vagabonde ; pour Christina, l'écriture est une fin en soi : non seulement sa vie tout entière se destine à l'écriture, mais elle dépend même entièrement de la réussite de son projet d'écrire.

Pour mieux comprendre ce caractère vital de l'écriture, il est nécessaire de percevoir son ambivalence : qu'elle puisse, tout à la fois, augmenter les souffrances de Christina et l'en guérir.

Christina vit dans la souffrance, une souffrance moins subie que recherchée. «Une chose », en effet, «était certaine : elle croyait à la souffrance. Elle la vénérait comme la source de toute grandeur $»($ p. 127). Christina est héritière du romantisme : pour elle, la souffrance est le signe d'une grande âme, et l'expression de la souffrance est la clé des grandes œuvres. Cependant, il est des moments où cette souffrance volontaire est si grande qu'elle en devient insupportable. Aussi, pour la calmer Christina a-t-elle parfois recours aux drogues - en particulier la morphine.

Or, l'écriture semble augmenter la souffrance de Christina et, corrélativement, sa dépendance à différentes formes de drogues. «Je l'avais vue à Neuchâtel pendant sa convalescence, la proie de son inspiration, cachant aux yeux des docteurs ses cigarettes et sa cafetière fidèle » (p. 41). Le portrait qu'Ella Maillart trace de Christina, au premier chapitre, commence et finit sur les mains de la jeune femme, comme si cette partie du corps, mieux que son visage, pouvait exprimer sa personnalité : or, si le passage se termine sur les mains de l'«artisan patient qui sait ciseler une ligne pure », il débute par la main qui tient une cigarette : "Maigre et longue, sa main aux articulations jaunes sous une peau en papier de 
soie tenait une cigarette américaine » (p. 24). C'est la même main qui tape à la machine et qui porte la cigarette aux lèvres : comme si l'écriture étaient étroitement liée au tabac.

Aussi, les médecins qui prescrivent à Christina une cure de repos se méfient-ils autant de l'écriture que de la cigarette ou du café. Or, ce qu'ils ne perçoivent pas, quand ils ne voient dans l'écriture qu'une menace, c'est qu'elle est un poison qui, pour Christina, contient aussi un remède - et qu'inversement, le remède qu'ils prescrivent à d'autres patients peut se révéler pour elle un poison : «ils ne voulaient pas comprendre qu'écrire était pour elle sa vie et sa nourriture, que la cure régénératrice de repos forcé imposée aux dyspeptiques ou aux hystériques ne pouvait pas lui convenir » (p. 57).

En effet, si l'écriture peut détériorer la santé de Christina, elle est aussi capable de la revigorer. D’une part, elle permet à Christina d'échapper à ses angoisses.

Mais voyons, lorsque vous écrivez un livre, qu'avez-vous de commun avec cet être plein de crainte que vous venez de décrire ?

- C'est pourquoi écrire a une telle importance pour moi. (p. 68)

Ainsi, d'une part, l'écriture permet à Christina d'oublier ses craintes en l'obligeant à se concentrer sur l'œuvre en cours. Cependant, d'autre part, elle peut aussi lui permettre de résoudre ses problèmes. Ella Maillart prend pour exemple la crise dans laquelle son mariage avec un diplomate nommé « chargé d'affaires » à Téhéran avait fini par plonger Christina :

Trois ans plus tard, elle se libéra enfin de son cauchemar persan en écrivant un long poème en prose. [...] Ce poème sensible et fiévreux l'épuisait tout en la renouvelant. «On se débarrasse de ses maladies dans ses livres, répétant et reproduisant à nouveau ses émotions afin de les maitriser », avait écrit D.H. Lawrence. Cela était vrai pour Christina. (p. 41)

Si l'écriture est associée à la souffrance, c'est que, dans un premier temps, elle en répète et reproduit les symptômes. Mais si l'écriture approfondit tout d'abord les tourments de Christina, elle lui permet aussi de les «maîtriser». Et c'est par l'écriture d'un « long poème en prose » qui lui fait tout d'abord revivre son «cauchemar persan », que Christina finit tout de même par s'en libérer.

L'écriture, si elle redouble tout d'abord la souffrance, permet finalement de s'en délivrer. Mais la souffrance est elle-même nécessaire à l'écriture - une souffrance d'autant plus grande, d'ailleurs, que Christina vise une écriture plus haute. Écriture et souffrance forment donc un cycle : pour écrire, il est nécessaire de souffrir et, pour cesser de souffrir, il est nécessaire d'écrire. C'est ce qu'Ella Maillart appelle la «voie cruelle de l'enfer » où 
Christina risque toujours, cependant, de ne pas arriver «à parfaire la courbe commencée, transformant ainsi l'enfer en paradis » (p. 127). En effet, si grande soit-elle, la souffrance ne garantit pas que le texte produit en soit à la hauteur, et qu'il parvienne à sublimer la souffrance en poésie.

Cependant, à lire les dernières lettres de Christina avant sa mort, Ella Maillart obtient la certitude que cette voie lui permit finalement d'atteindre son objectif littéraire. En effet, il semble bien que Christina parvienne à dissocier l'écriture de toute forme de dépendance : «Maintenant, je le sais, le tabac ou la drogue sont inutiles. Sans eux je me tourne vers une autre concentration, plus proche de ce dont j'ai besoin pour écrire » (p. 311). La main de l'écrivain cesse alors d'être celle qui tient la cigarette, pour n'être plus qu'une main d'artisan glissant des feuilles dans une machine à écrire.

Mais, selon Ella Maillart, la réussite de Christina n'a pas qu'un aspect littéraire : «Non seulement vous êtes parvenue à vous exprimer, Christina, mais vous avez encore éprouvé le plus profond de vous-même, le Centre, ayant enfin réussi à dépasser le tourment qui vous ravageait » (p. 75). Aussi, la voie cruelle de Christina ne lui permet pas seulement d'écrire; elle lui permet aussi d'atteindre ce Centre - cette personnalité, cette note fondamentale - que recherche aussi Ella Maillart.

Mais en recherchant la souffrance, elle accomplissait beaucoup; elle amoindrissait sans cesse cet ego qui, réclamant toujours des jouissances, empêche chez chacun de nous l'épanouissement de notre personnalité. Désirer la douleur est contraire aux exigences habituelles de l'ego. [...] Elle vécut le plus profond en elle, ce qu'il y a de plus précieux, l'inaltérable vérité ; pour finir, cette transcendance l'illumina, mais je ne sais pas si sa vie journalière eut le temps d'en être transformée. (p. 310)

Les souffrances que s'imposent Christina ont pour effet d'user l'ego qui se complaît plutôt dans les plaisirs. Et, à force de souffrances, Christina parvient même à s'en débarrasser, permettant alors à «notre personnalité » de s'épanouir. Ainsi, la voie cruelle ne conduit pas seulement Christina à l'écriture : elle lui permet de vivre «le plus profond en elle ». Sans peut-être le savoir, Christina poursuit donc le même but que Kini, même si les deux amies divergent sur les moyens - ou plutôt sur les voies - pour l'atteindre : « je fais le siège de cette plénitude dans la joie », écrit Ella Maillart, « et non pas dans un gouffre de peine » (p. 128).

Ella Maillart se rappelle d'ailleurs que, lors de leur première rencontre, on pouvait déjà deviner à certains détails ce que Christina cherchait vraiment. 
C'était par une claire journée d'été. Sans chapeau, chic dans un tailleur gris, si mince qu'elle en était presque éthérée, assise, elle demeurait silencieuse et penchée. Et soudain un sourire confiant s'esquissait. D'abord il me sembla qu'elle écoutait une musique intérieure. Ensuite je sentis qu'elle cherchait. Elle écoutait au-delà des mots prononcés, dans l'espoir d'y saisir comme une résonance d'un monde doté de plus de sens que le nôtre : elle attendait la note fondamentale. (p. 126)

Il s'agit d'un portrait assez inattendu : en effet, dans tout le récit, Christina est décrite comme un être de souffrance; or, lors de cette première rencontre, elle apparaît non seulement calme, mais souriante. «Presque éthérée », elle n’est pas tout à fait dénuée de poids, et fait donc encore partie de ce monde ; cependant, son «sourire confiant » montre que, même si elle ne perçoit pas encore, derrière le silence, la « résonance d'un monde doté de plus de sens que le nôtre ", elle éprouve la tranquille certitude d'y parvenir un jour. Elle est attentive : elle prête attention et attend. Aussi, sa posture, «assise », «silencieuse », évoque celle de la méditation; et l'esquisse d'un sourire semble annoncer la béatitude à laquelle Christina aboutit finalement. La souffrance n'est donc pas seulement une voie qui mène à l'écriture, elle est aussi ce qui permet de libérer « une musique intérieure ». Mais n'est-ce pas, finalement, une seule et même chose : et vouloir écrire, n'est-ce pas vouloir capter par le langage l'expression de notre «note fondamentale »?

Ainsi, l'échec du voyage à guérir Christina de sa dépression confirme Ella Maillart dans ce qu'elle pressentait déjà : «le monde est moins réel que ce qui active notre vie intérieure » (p. 42). Et, de même que le remède aux maux de l'Europe n'est pas dans un Éden asiatique, ni dans une tribu édénique, de même, le remède aux maux de Christina n'est pas dans l'admiration du monde extérieur : l'un et l'autre ne sauraient être trouvés que dans la « vie intérieure ». Par conséquent, si Ella Maillart a quitté 1'Europe, ce n'est pas vraiment pour se rendre en Asie. «Loin d'une tremblante et fiévreuse Europe, je voulais simplement tourner mes regards en moi-même » (p. 59). Ce qu'elle cherchait dans l'espace du dehors, se trouve en fait dans l'espace du dedans. L'Éden qu'elle situait dans un lointain géographique, est en fait localisé dans un lointain intérieur. Comme l'écrit Ella Maillart dans une lettre adressée à sa mère en 1941 : «C'est en soi que chacun doit trouver son équilibre et son âge d'or » (MAILLART, 2003 : 152). À la région centrale de l'Asie, vers laquelle tendaient tous les voyages, Ella Maillart substitue un «Centre» qui se situe «en chacun de nous ». Aussi, 
peut-elle pleinement donner à son récit un titre qui relève moins d'un itinéraire - comme pouvait l'être, par exemple, Des Monts célestes aux sables rouges - que d'un parcours intérieur. Et affirmer, dès la couverture, la victoire de Christina sur Kini - de l'écrivain sur la voyageuse : en effet, alors qu'Ella Maillart prétendait guérir Christina de sa souffrance par le voyage, c'est cette dernière qui, finalement, guérit Kini des voyages, en lui montrant que, pour atteindre le Centre, la souffrance et l'écriture tracent une «voie » peut-être «cruelle», mais néanmoins capable de conduire à destination plus sûrement que n'importe quel voyage. 


\section{CONCLUSION : La Voie littéraire}

Ainsi, La Voie cruelle peut être lu comme un récit de la fin des voyages, aussi bien au niveau de l'œuvre d'Ella Maillart que, plus largement, dans l'histoire du genre.

D’une part, en effet, le récit annonce la fin de la diversité culturelle face à l'expansion d'une civilisation industrielle dont les moyens de transports modernes sont les propagateurs. L'Ailleurs devient de plus en plus semblable à l'Ici, et l'Autre, au voyageur. Ella Maillart se trouve en quelque sorte à un tournant des voyages où, à l'émerveillement devant la diversité des paysages et la variété des cultures, qui depuis Marco Polo fonde le rapport du voyageur au monde, vient peu à peu se substituer le triste constat d'une uniformité sordide.

Or, c'est dans une humanité préservée de l'influence européenne qu'Ella Maillart espérait trouver, en cette veille de Seconde Guerre Mondiale, le remède aux maux dont souffre l'Occident. Car voyager, ce n'est pas seulement aller au loin, c'est aussi remonter dans le temps : c'est trouver dans une culture lointaine le reflet préservé de sa propre culture. Cependant, contrairement à l'Orient du Moyen-Âge et de l'époque classique, il n'est plus possible d'espérer trouver, dans l'Orient du XXe siècle, un jardin d'Éden abritant une humanité originelle, ni même une tribu édénique errant parmi les nomades : comme Ella Maillart le découvre en Afghanistan, les ingénieurs ont envahi le Paradis, et les derniers nomades sont en voie d'extinction. L'Éden demeure à jamais inaccessible : non pas qu'il soit défendu par des murailles infranchissables, mais parce qu'il semble bien que rien, sur terre, ne puisse vraiment échapper à la civilisation européenne.

Aussi, La Voie cruelle est une sorte d'adieu au vieux monde des voyages, dont le récit parvient encore, dans certains paysages et certaines peuplades, à capter les derniers reflets, avant qu'ils ne disparaissent définitivement. Et, l'on pourrait élargir à ce monde en déclin cette remarque d'Ella Maillart à propos des ruines d'Herat, en Iran :

Préférons-nous ce qui est sur le point de disparaitre ? Le Parthénon serait-il aussi émouvant avec ses peintures et dorures encore intactes ? Temps, guerre et secousses sismiques ont abîmé Herat, mais le peu qui reste - une tombe et des minarets dans un champ de blé - me touche comme le dernier sourire d'un ami. (p. 174) 
Or, c'est bien un « dernier sourire » que le monde des voyages, lui aussi « sur le point de disparaître », adresse à Ella Maillart au long de sa route, conférant aux pages de La Voie cruelle un ton nostalgique. Aussi, Ella Maillart n'est pas seulement l'héritière d'une tradition des voyages : elle en est aussi l'une des dernières représentantes, et La Voie cruelle qui, sous bien des aspects, est son dernier récit de voyage ${ }^{17}$, témoigne d'une sorte de « désenchantement » du monde.

La perte du Paradis, que les voyageurs plaçaient au cœur de l'Asie ou parmi les îles du Pacifique, conduit alors Ella Maillart à remettre en question l'utilité des voyages.

C'était peut-être parce que j'étais libérée de ma fièvre de voir «le pays caché par l'horizon ». Lhassa ou Papeete auraient pu surgir devant moi sans que mon cœur se serre d'émotion. Je n'étais plus fière d'avoir réussi par mes seuls efforts à transformer le monde en un terrain de jeu à ma merci. (p. 288)

Tous ces précédents voyages, que ce soit sur mer ou en Asie, avaient tendu vers un Paradis : Papeete ou Lhassa. Or, ce voyage en Afghanistan lui fait perdre non seulement tout espoir de trouver «le pays caché par l'horizon », mais aussi tout intérêt pour ce dernier : il pourrait surgir devant elle, qu'elle n'en serait pas autrement émue. Car ce voyage lui enseigne que le vrai Paradis n'est pas situé dans une géographie extérieure : il faut le chercher dans la géographie intérieure.

Je commençais alors à découvrir ce que l'avenir ne ferait que confirmer : pour la première fois, le voyage dans le monde objectif ne parvenait plus à me captiver entièrement. Car le monde est moins réel que ce qui active notre vie intérieure. (p. 42)

La disparition de la diversité des cultures sous une seule et même civilisation, non seulement détourne Ella Maillart du monde extérieur - le «monde objectif »-, mais oriente ses recherches vers «la vie intérieure ». Ce qu'elle avait découvert en voyageant avec Peter Fleming, et que confirme son voyage avec Annemarie Schwarzenbach, c'est que le voyage dépend moins des lieux qu'on traverse que du regard du voyageur: en effet, selon la sensibilité et l'humeur de ce dernier, le monde qui l'entoure apparaîtra de manière très

\footnotetext{
${ }^{17}$ Publié en anglais en 1947, et traduit par l'auteur elle-même en 1951, c'est le dernier livre écrit - ou du moins ré-écrit - par Ella Maillart en français.
} 
différente ; si bien que deux personnes peuvent se trouver ensemble au même endroit, elle ne feront pas pour autant le même voyage.

Aussi, à quoi bon continuer d'écrire sur le monde extérieur, si « le monde est moins réel que ce qui active notre vie intérieure »? À quoi bon continuer d'écrire des récits de voyage ? À quoi bon vouloir donner aux lecteurs «le goût du monde »?

Pourquoi ne pas écrire des livres qui donnent le goût du monde à ceux qui sont enchaînés?

- Si mes livres ont atteint un tel objectif, cela ne me satisfait plus, répondisje. À quoi cela sert-il d'envoyer des gens de par le monde? Je sais, d'expérience, que courir le monde ne sert qu'à tuer le temps. On revient aussi insatisfait qu'on est parti. (p. 262)

La remise en question de la réalité du monde et de l'utilité des voyages conduit ainsi Ella Maillart à interroger sa propre pratique de l'écriture. Si l'important est en chacun de nous, s'il s'agit de trouver un Centre qui ne soit plus géographique mais intime, il n'y a plus aucune raison d'écrire des récit de voyages. Il faudrait écrire non plus des récits d'exploration, mais des récits d'introspection, qui engagent les lecteurs non pas à «courir le monde », mais à entrer en eux-mêmes.

Ainsi, dans La Voie cruelle, la fin des voyages - et, corrélativement, celle des récits des voyageurs - est-elle la conséquence, d'une part, de l'expansion d'une civilisation unique et, d'autre part, d'un renversement qui accorde au monde subjectif une réalité plus grande qu'au monde objectif. Cependant, Ella Maillart ne met pas seulement un terme aux récits de voyage : elle en montre aussi une voie possible.

D’une part, Ella Maillart préconise un récit qui accorde plus de place au sujet qu'à l'objet - au voyageur qu'au pays visité. Et d'ailleurs, au XXe siècle où le monde n'est plus à découvrir - surtout s'il tend à devenir partout le même - l'important n'est plus tellement ce que regarde le voyageur que le regard qu'il porte. Et le lecteur de récits de voyage s'inquiétera moins, aujourd'hui, du pays de destination que de son compagnon de voyage. En effet, lit-on La Voie cruelle pour l'Afghanistan ou pour Ella Maillart ? Et qui lit, par exemple, Chronique japonaise ou L'Empire des signes, cherche moins des informations sur le Japon forcément partielles, forcément datées - qu'à partir en compagnie de Nicolas Bouvier ou de Roland Barthes. Car s'ils prennent le même pays pour destination, à la même époque, l'un et l'autre n'invitent pas le lecteur au même voyage.

D'autre part, Ella Maillart montre que s'il s'agit d'atteindre ce qu'elle appelle le Centre, de faire entendre la note intérieure, alors il est nécessaire, à l'exemple d'Annemarie 
Schwarzenbach, d'adopter une écriture littéraire : une écriture qui s'inquiète moins de dire le vrai sur le monde, que de faire sonner les mots de la manière la plus juste. Il est une voie cruelle, qui est la voie littéraire, où grâce au dépassement de la souffrance par l'écriture, on peut faire sauter l'ego individuel et libérer notre personnalité commune. Cependant, cette voie littéraire est loin d'être évidente pour le récit de voyage : en effet, le genre s'est construit en marge de la littérature - sinon contre. Et désigner aux récits de voyage la littérature comme voie possible de survie risque peut-être au contraire d'en sonner l'arrêt de mort.

Voyageurs qui écrivent. En effet, contrairement à ce que laisse entendre l'expression «littérature de voyage » - par laquelle on désigne tout type de relations de voyage, qu'elles prennent la forme de récits, de carnets de routes, ou encore de lettres - littérature et voyage ne font pas forcément bon ménage. Il a même fallu du temps pour que la littérature de voyage soit reconnue comme genre littéraire. Comme le fait remarquer Fernando Cristovão, « foi tardio e é relativamente recente o reconhecimento do subgênero Literatura de Viagens devido, sobretudo, à sua natureza compósita e interdisciplinar. »(CRISTOVÃO, 199 : 16). Et l'on peut en effet attribuer cette reconnaissance tardive à des œuvres qui relèvent autant (ou parfois, moins) de la littérature, que de la géographie, de l'histoire, ou de l'anthropologie.

Cependant, on peut aussi trouver des raisons à ce retard non plus seulement dans la nature («composite ») des œuvres, mais aussi dans celle de leurs auteurs. En effet, la plupart des auteurs de relations de voyage, du XVIe au XVIIIe siècles, ne font pas partie de l'institution littéraire. Ce ne sont pas des écrivains, ni des philosophes : comme le regrette Jean-Jacques Rousseau, dans la Note X du Discours sur l'origine et les fondements de l'inégalité parmi les hommes, il n'y a «guères que quatre sortes d'hommes qui fassent des voyages de long cours; les Marins, les Marchands, les Soldats et les Missionnaires» (ROUSSEAU, 1969 : 142). Aussi, en relatant leurs voyages, ces derniers n'ont-ils pas pour objectif de faire une œuvre littéraire, mais de transmettre un savoir sur les régions qu'ils ont visitées. Ils sont d'ailleurs les premiers, très souvent, à dénier toute aspiration au métier d'écrivain, et à reconnaître leur manque de talent pour la littérature. Ainsi, Pero Vaz de Caminha, en 1500, commence-t-il la Lettre qu'il adresse au roi de Portugal pour lui rendre compte de la découverte du Brésil en s'excusant de son peu de talent pour écrire un tel récit :

Posto que o Capitão desta Vossa frota e assim igualmente os outros capitães escrevam a Vossa Alteza dando notícias do achamento desta Vossa terra nova, que agora nesta navegação se achou, não deixarei de também eu dar minha conta disso a Vossa Alteza, fazendo como melhor me for possível, 
ainda que - para o bem contar e falar - o saiba pior que todos. Queira porém Vossa Alteza tomar minha ignorância por boa vontade, e creia que certamente nada porei aqui, para embelezar nem para enfeiar, mais do que vi e me pareceu. (CAMINHA, $1985: 88$ )

Quoique qu'il promette de faire de son mieux, Pero Vaz de Caminha s'avoue non seulement peu doué pour «bien raconter et dire »son voyage, mais encore, à l'en croire, de tous ceux qui ont accompagné Cabral, il serait le moins doué pour le faire. Il est évident que pareil aveu tient avant tout des convenances, et que pour des raisons hiérarchiques, Caminha ne saurait se considérer du même niveau que ses «capitaines », et encore moins digne de l'autorité suprême à laquelle il s'adresse. Cependant, il tourne l'aveu de ses limites à l'avantage de son récit, car son « ignorance » témoigne, selon lui, de sa «bonne volonté », et surtout offre un gage de meilleure adéquation de son texte au vécu : en effet, faute d'un vrai talent d'écrivain, il ne saurait rien «embellir» ni «enlaidir», se trouvant donc limité à ne parler que de ce qu'il a effectivement « vu »- sans en rajouter - ou, du moins, de ce qu'il lui a «semblé » voir. Aussi Caminha tire-t-il parti de ses (soi-disant) piètres talents d'écrivain, en les présentant comme autant de gages de sincérité.

Montaigne, au début de son essai «Des Cannibales », résume fort bien les attentes du XVIe siècle à l'égard des récits de voyageurs : en premier lieu, qu'ils ne tirent pas d'un voyage effectué dans une partie précise du monde le droit abusif de parler du monde entier.

Il nous faudrait des topographes, qui nous fissent narration particulière des endroits où ils ont été. Mais pour avoir cet avantage sur nous, d'avoir vu la Palestine, ils veulent jouir du privilège de nous conter nouvelles de tout le demeurant du monde. (MONTAIGNE, 2007 : 211)

Derrière le voyageur qui, parce qu'il a «vu la Palestine », s'autorise à «conter nouvelles du reste du monde », Montaigne désigne sans aucun doute André Thevet. En effet, au retour d'un voyage en Palestine, en 1554, ce dernier avait publié une Cosmographie de Levant qui, comme Les Singularités de la France Antarctique, sera finalement absorbée dans sa volumineuse Cosmographie universelle. De la cosmographie du Levant à celle de l'univers, on comprend que Montaigne ait pu juger que son contemporain tirait de ses voyages un «privilège » disproportionné. En cela, Jean de Léry est certainement plus proche de ce que Montaigne appelle un «topographe »: un voyageur qui se propose de rendre compte d'un espace strictement délimité par sa propre expérience. Léry s'en tiendra à ce qu'il a «pratiqué, veu, ouy et observé » (LÉRY, 1994: 105), et son Histoire correspond certainement davantage à cette « narration particulière » que Montaigne appelle de ses vœux. 
Cependant, les exigences de ce dernier à l'égard des voyageurs ne portent pas seulement sur le contenu de leurs récits : s'il attend d'eux qu'ils s'en tiennent strictement à ce qu'ils savent par expérience, il souhaite plus encore qu'ils usent d'un langage simple, voire même fruste. Aussi prêtera-t-il plus attention aux récits d'un «homme simple et grossier » qu'à celui de «fines gens ».

Cet homme que j'avais était homme simple et grossier, qui est une condition propre à rendre véritable témoignage : car les fines gens remarquent bien plus curieusement, et plus de choses, mais ils les glosent : et pour faire valoir leur interprétation, et la persuader, ils ne se peuvent garder d'altérer un peu l'histoire : ils ne vous représentent jamais les choses pures; ils les inclinent et masquent selon le visage qu'ils leur ont vu : et pour donner crédit à leur jugement, et vous y attirer, prêtent volontiers de ce côté-là à la matière, l'allongent et l'amplifient. Ou il faut un homme très fidèle, ou si simple, qu'il n'ait pas de quoi bâtir et donner de la vraisemblance à des inventions fausses [...]. (MONTAIGNE, $2007: 210-211$ )

Pour Montaigne, la simplicité est un gage d'authenticité, et trop de raffinement, au contraire, pourrait prêter au doute. Bien qu'il reconnaisse aux hommes cultivés, «fines gens », un meilleur sens de l'observation, Montaigne se méfie néanmoins de leur tendance à «gloser»: soit qu'ils veuillent imposer leur vision des choses (au lieu de s'en tenir aux choses vues), qu'ils veuillent les transformer, ou pire encore, en inventer de toutes pièces, ceux qui maîtrisent l'art de la parole sauront toujours trouver des mots capables d'appuyer leur point vue, et de donner de la «vraisemblance » à leurs inventions, sans qu'on puisse démêler le vrai du faux. Au contraire, un homme «simple et grossier», sans éducation, ne saurait s'en tenir qu'aux faits bruts, aux « choses pures », faute de talent pour les amplifier ou en imaginer d'autres; et si jamais il lui prenait de vouloir mentir, il se dénoncerait par la grossièreté de ses mensonges, et l'on pourrait toujours séparer dans ses propos la vérité crue de l'invention maladroite.

La méfiance de Montaigne porte donc moins, finalement, sur les inventions auxquelles un voyageur peut se prêter, que sur son aptitude à leur donner un tour convainquant. L'auteur des Essais craint moins le mensonge que la rhétorique, le menteur que le sophiste. Aussi, un lettré qui relate un voyage est-il sujet à caution : il risquerait d'en profiter pour faire de la littérature. Quant il s'agit de voyage, Montaigne préfère un témoignage, aussi maladroit soitil, à une œuvre littéraire.

Et c'est probablement une des raisons pour lesquelles, de la Renaissance au Siècle des Lumières - et encore même au XXe siècle - les auteurs de relations de voyage se tiennent volontairement à distance de la littérature, affirmant leur peu d'aptitude pour les Belles- 
Lettres : c'est qu'ainsi, ils donnent plus de crédit à leurs témoignages, toute prétention littéraire pouvant être regardée comme une tentation d'«embellir» le récit, de déformer les faits. La littérature n'est jamais loin de la fiction, ni l'écrivain du conteur - pour ne pas dire du menteur. S'en démarquer, cela permet d'éloigner les soupçons de mensonge, et de créditer ses écrits d'une plus grande authenticité. C'est bien d'une relation qu'il s'agit, et non pas d'une fiction. Nous sommes dans la relation d'un voyage réel, non pas dans celle d'un voyage imaginaire. Ainsi, de Bougainville, s'excusant auprès de ses lecteurs que «l'habitude d'écrire » n'ait pu lui « apprendre à sauver par la forme une partie de la sécheresse du fond » (BOUGAINVILLE, 1997 : 18-19) », jusqu’à Ella Maillart, affirmant à son éditeur : «je ne suis pas écrivain » (MAILLART, 2001b : 74), la plupart des voyageurs qui écrivent, non seulement ne se considèrent pas des écrivains professionnels, mais refusent même ouvertement toute prétention littéraire.

Écrivains qui voyagent. À partir du XIXe siècle, cependant, les écrivains se mettent à voyager eux aussi. En effet, les voyages ne sont plus réservés, comme ils pouvaient l'être encore au XVIIIe, aux marins, aux marchands ou aux aventuriers ; ils s'ouvrent à un plus large public, dans lequel on compte de plus en plus d'auteurs confirmés. Aussi ces derniers ne se contentent plus, comme le regrettait Rousseau, de gloser à partir du voyage des autres : ils se mettent à relater leurs propres voyages. La relation de voyage entre dans les pratiques littéraires.

Cependant, il semble que l'écrivain qui entreprend de relater un voyage se trouve placé devant un dilemme : ou bien il continue de faire de la littérature, mais dans ce cas, il n'écrit pas une véritable relation de voyage ; ou bien il se met à écrire une vraie relation, mais il lui faut alors abandonner toute prétention littéraire. L'exemple de deux écrivains du XXe siècle, Paul Claudel et André Gide, illustre bien chacune des deux alternatives.

Comme le remarque Claude-Pierre Perez «Claudel a été sa vie durant le client assidu des lignes transocéaniques »; néanmoins, «il n'a pas laissé de volume qu'on puisse inscrire sans hésiter à la rubrique des récits de voyage » (HAMBURSIN, 2005 : 19).

Claudel ne se soucie pas d'être un chroniqueur, ni de faire du « reportage », comme disait avec dédain Mallarmé, il n'a conclu aucun «pacte autobiographique », aucun « contrat de véridicité » ou autre. Le réel, le vu, le vécu, servent ici de tremplin, de planche d'appel, de pont d'envol à ce qu'il faut bien appeler une fiction. (HAMBURSIN, $2005: 22$ ) 
Connaissance de l'Est est un des « deux livres où l'on peut trouver quelque chose qui rappelle d'un peu près ce que l'on trouve d'habitude sous l'étiquette de travel writing ». Et, prenant un exemple parmi les poèmes de ce recueil, Claude-Pierre Perez montre alors comment dans «La Maison suspendue », où Claudel évoque un ermitage bouddhiste du nom de Yong-Fou (un temple construit dans une vaste grotte à flanc de montagne), le poète passe rapidement du récit de l'excursion à la rêverie, s'identifiant aux bonzes, s'imaginant une vie recluse, anticipant l'enfermement monastique auquel, par vocation religieuse, il s'était luimême destiné : « il s'agit de devenir moine, il s'agit, sautant sur cette occasion que procure la caverne chinoise, de faire une expérience imaginaire de la claustration » (HAMBURSIN, 2005 : 22). Le temple bouddhique est donc moins l'objet d'une description, comme dans un récit de voyage, qu'il ne sert de support à une rêverie poétique, de «tremplin » à une «fiction ».

L'exemple de Claudel confirme ainsi ce qu'écrit Henri Michaux dans Passages : «les poètes voyagent, mais l'aventure du voyage ne les possède pas » (MICHAUX, 2001 : 307). Et, de même qu'il semble que les poètes soient peut doués pour les voyages, de même, il semble que la «passion des voyages n'aime pas les poèmes ». Michaux affirme que la prose lui serait plus adaptée. Cependant, ce n'est pas dans la prose la plus littéraire que, selon lui, on trouve «la poésie du voyage » : en effet, elle préfère «le style moyen et le mauvais, et même s'y exalte ».

S'il lui arrive d'avoir grande allure dans Chateaubriand ou dans quelque autre seigneur de la littérature, elle trouve plus souvent sa note juste, et qui vous frappe, dans un marchand, un aventurier, un embrouilleur aux cent métiers, qui la transpire et révèle en quelque naïf propos qu'Elle le tient souverainement.

[...] En somme. Elle préfère la mauvaise compagnie. (MICHAUX, 2001 : 307)

Aussi, guère plus que le poète, le grand écrivain - le «seigneur de littérature » - ne serait indiqué pour écrire un récit où l'on retrouve la «note juste » du voyage. Au contraire, toute autre personne moins rompue aux techniques de la littérature et aux beautés du style, un «marchand» ou un «aventurier», la rendra mieux dans son propos «naïf ». Aussi bien Montaigne qu'Henri Michaux s'accordent donc sur ce point : le langage littéraire n'est pas le plus approprié pour exprimer le voyage. Et si les écrivains qui ont relaté leur voyage ont pu produire de la bonne littérature, ce ne serait pas pour autant de la bonne littérature de voyage. 
D'ailleurs, au cours de son voyage au Congo, dont il tient scrupuleusement le journal, André Gide constate que les conditions matérielles du voyage ne se prêtent guère à l'écriture littéraire. Relatant un dîner auquel il vient de participer, il regrette de ne pouvoir qu'en « rapporter à peu près les propos », et surtout de ne pouvoir en rendre mieux «l'atmosphère inquiétante, fantastique »: «on ne pourrait y arriver », écrit-il, «qu'avec beaucoup d'art; et j'écris au courant de la plume »(GIDE, 1928 : 139-140). La tenue d'un journal de voyage implique en effet qu'on écrive dans une sorte d'urgence : en effet, d'une part, le voyage ne s'arrête pas, apportant chaque jour son lot d'impressions nouvelles, de rencontres, de paysages, qu'il s'agit de retranscrire à mesure, pour ne rien en perdre ; d'autre part, le voyage occupe l'essentiel du temps, et limite celui qu'on peut accorder à l'écriture. Alors que l'écriture littéraire exige qu'on puisse s'attarder plusieurs jours sur une page - qu'on prenne beaucoup de temps pour écrire peu -, l'écriture du voyage (on pourrait dire l'écriture « viatique ») exige au contraire qu'on écrive beaucoup en peu de temps.

Au début, le voyage d'André Gide était pourtant éminemment littéraire. En dédiant son journal à Joseph Conrad, il montre assez combien l'auteur anglais, dont Gide fut aussi le traducteur, est a l'origine de son départ. Et, autant et plus que le Congo véritable, c'est celui d'Au Cour des ténèbres que Gide semble chercher à chaque détour de la forêt africaine : «le spectacle se rapproche de ce que je croyais qu'il serait», écrit-il dans son journal, le 7 septembre, «il devient ressemblant »(GIDE, 1928 : 43). Cependant, à mesure qu'il découvre le Congo véritable et, en particulier, les injustices de la colonisation française, Gide donne à son voyage un tour moins littéraire et plus engagé.

Désormais, une immense plainte m'habite ; je sais des choses dont je ne puis pas prendre mon parti. Quel démon m'a poussé en Afrique ? Qu'allais-je donc chercher dans ce pays? J'étais tranquille. À présent je sais; je dois parler.

Mais comment se faire écouter? Jusqu'à présent, j'ai toujours parlé sans aucun souci qu'on m'entende ; toujours écrit pour ceux de demain, avec le seul désir de durer. J'envie ces journalistes dont la voix porte aussitôt, quitte à s'éteindre sitôt ensuite. (GIDE, 1928 : 113)

Gide est habité d'une « plainte », qu'il lui faut absolument crier. Cependant, il lui faut non seulement «parler », mais encore « se faire écouter ». Or, l'écriture littéraire à laquelle des années de pratique l'ont rompu, Gide reconnaît qu'elle n'est pas destinée à ses contemporains, mais «à ceux de demain». Dans son «désir de durer», peu importe à l'écrivain d'être lu. Cependant, le voyage le place devant une réalité qu'il lui faut impérativement révéler à ses contemporains. Il faut qu'il soit lu, quitte à ce qu'on l'oublie 
«sitôt ensuite». Aussi éprouve-t-il la nécessité d'abandonner l'écriture littéraire, pour se rapprocher d'une écriture plus journalistique.

Par conséquent si Gide admet d'une part que le voyage n'est pas adapté à l'écriture littéraire, d'autre part, à mesure qu'il découvre le Congo et la société coloniale, il reconnaît que l'écriture littéraire n'est pas non plus la mieux adaptée pour dire le voyage. Le voyage semble conduire à un autre type d'écriture.

Ainsi, il y aurait d'une part l'écriture littéraire, que certains écrivains en voyage comme Claudel - continueraient de pratiquer, au risque de perdre le voyage ; et d'autre part, une écriture viatique, pratiquée par des voyageurs sans talent littéraire - ainsi que par des écrivains se résignant à pratiquer une écriture différente que celle qu'ils pratiquent habituellement -, et qui seule parviendrait à dire le voyage. Aussi, l'expression littérature de voyage semble-t-elle contradictoire, puisqu'il faudrait choisir entre une littérature impropre au voyage et une écriture du voyage impropre à la littérature. Il semble bien qu'écriture littéraire et écriture viatique ne puissent coïncider.

Écrivants et écrivains voyageurs. Dans sa préface à la réédition d'Oasis interdites, intitulée «Ella Maillart et la Chine du Nord», Nicolas Bouvier distingue bien les deux types d'auteurs de relations de voyage, affirmant lui aussi - après Montaigne et Michaux -

qu'on a souvent plus de profit à lire les voyageurs qui écrivent que les écrivains qui voyagent. La véritable «connaissance de l'Est» n'est pas toujours du côté que l'on croit et s'il s'agit de faire un bout de route en Asie, je préfèrerai de beaucoup la compagnie d'Ella Maillart à celle de Paul Claudel. (BOUVIER, $2004:$ 1065)

Reprenant l'opposition entre le poète (cet écrivain superlatif) et le voyageur, entre poésie et relation de voyage, Bouvier conteste donc à Paul Claudel le titre choisi pour son recueil, et place « la véritable connaissance de l'Est » du côté d'Ella Maillart et de ses récits. Il vaut mieux lire les voyageurs, semble-t-il, si l'on veut connaître un pays, ses habitants, ses routes : si l'on cherche un savoir.

La distinction proposée par Nicolas Bouvier entre «voyageurs qui écrivent»et « écrivains qui voyagent »n'est pas sans rappeler celle que proposait Roland Barthes, dans les Essais critiques, entre écrivants et écrivains.

Les écrivants, selon Barthes, «sont des hommes transitifs; ils posent une fin (témoigner, expliquer, enseigner) dont la parole n'est qu'un moyen ; pour eux, la parole supporte un faire, elle ne le constitue pas » (BARTHES, 1964 : 156). Si l'écrivant «apporte 
quelque attention à l'écriture, ce soin n'est jamais ontologique : il n'est pas souci ». Si l'écrivant manifeste un « soin » dans le choix des mots, c'est pour qu'ils ne trahissent pas sa pensée, pour qu'il n'y ait pas d'ambiguïté ; ce n'est pas dans un but esthétique, ni par «souci » du style.

Par contre, pour l'écrivain, le style l'emporte sur l'information à transmettre. Pour lui, le travail sur le langage importe plus que le sujet dont il traite. Selon Barthes, l'écrivain « est un homme qui absorbe radicalement le pourquoi du monde dans un comment écrire » (BARTHES, 1964 : 154). Pour l'écrivain, « la parole est une matière (infiniment) travaillée ; elle est un peu comme une sur-parole, le réel ne lui est jamais qu'un prétexte (pour l'écrivain, écrire est un verbe intransitif); il s'ensuit qu'elle ne peut jamais expliquer le monde ». Pour l'écrivain, écrire est une activité en soi, refermée sur elle-même, narcissique : peu lui importe d'écrire sur le monde (encore moins de l'expliquer), il écrit tout court. Si l'écrivain malgré tout, retrouve le monde, c'est en bout de course, après lui avoir nier tout intérêt et préféré le langage.

Et le miracle, si l'on peut dire, c'est que cette activité narcissique ne cesse de
provoquer, au long d'une littérature séculaire, une interrogation au monde :
en s'enfermant dans le comment écrire, l'écrivain finit par retrouver la
question ouverte par excellence : pourquoi le monde ? Quel est le sens des
choses? En somme, c'est au moment même où le travail de l'écrivain
devient sa propre fin, qu'il retrouve un caractère médiateur : l'écrivain
conçoit la littérature comme fin, le monde la lui renvoie comme moyen : et
c'est dans cette déception infinie, que l'écrivain retrouve le monde, un
monde étrange d'ailleurs, puisque la littérature le représente comme une
question, jamais en définitive, comme une réponse. (BARTHES, 1964:154)

Alors que l'écrivant se sert du langage pour expliquer le monde, l'écrivain finit par interroger le monde par le langage. Si le premier donne des réponses, le deuxième pose des questions. Si le premier lève les ambiguïtés, l'autre les multiplient.

Aussi l'on comprend mieux que la connaissance ne soit pas du côté de l' «écrivain qui voyage ». Même si, au départ, sont projet était d'écrire une relation de voyage qui rendît compte du monde, non seulement son projet d'écriture le ramène au langage, l'arrête aux mots, mais l'œuvre qu'il produit, bien loin d'expliquer le monde, finit en fait par le questionner. Sous la plume de l'écrivain, le monde ne devient pas plus connu; au contraire, il devient plus « étrange ».

Par contre, les « voyageurs qui écrivent » sont plutôt des écrivants, au sens où l'entend Roland Barthes, dans la mesure où leur préoccupation porte avant tout sur les informations qu'ils souhaitent transmettre. Aussi, la langue qu'ils utilisent n'est pas une fin, mais juste un 
moyen. L'important, en effet, est d'élargir la connaissance du monde : pour eux, le réel ne saurait être un prétexte, mais bien ce qu'il s'agit de décrire et d'expliquer.

Ainsi, en reprenant d'un point de vue barthésien la distinction proposée par Nicolas Bouvier, nous trouverions aux côtés d'écrivants voyageurs, pour qui l'écriture est au service d'un savoir sur le monde, des écrivains voyageurs, pour qui le monde ne serait qu'un prétexte et l'écriture une fin. C'est cette même distinction que propose aussi Ella Maillart dans La Voie cruelle, en plaçant aux côtés de Kini-l'écrivant, pour qui l'écriture est un moyen - dont elle tire d'ailleurs l'argent nécessaire à ses voyages - Christina-l'écrivain, pour qui l'écriture, au contraire, est une fin, l'objectif ultime vers lequel tendent tous ses efforts.

Écrivain-voyageur. Si, comme on le notait en introduction, à partir des années soixante-dix, la littérature de voyage connaît un second souffle - remettant à l'honneur l'œuvre d'Ella Maillart -, il semble bien qu'elle se soit de nouveau affirmée contre la littérature tout court. En effet, certains de ses défenseurs brandissent la littérature de voyage contre de soi-disant excès de la littérature.

$\mathrm{Au}$ nombre des griefs que Michel Le Bris, chef de file de ces nouveaux écrivainsvoyageurs, énonce contre la littérature, il y a tout d'abord l'absence du monde qui, selon lui, aurait été ignoré des écrivains français de l'après-guerre - en particulier ceux du Nouveau Roman - au nom d'une esthétique trop éprise de forme et de linguistique.

Nous savons, aujourd'hui un peu mieux qu'hier, de quoi meurt la littérature : de s'être faite la servante des idéologies, sous le prétexte de l'engagement, de se noyer dans le trop-plein de soi, sous le prétexte de psychologie, ou, à l'inverse, de se satisfaire de n'être plus que « littérature »: jeux de mots. Lui reste peut-être, pour retrouver son sens, ses énergies, après des décennies d'asservissement au Signe-Roi, à retrouver le monde. (COLLECTIF, 1992 : 140)

Le monde d'un côté ; une littérature narcissique, de l'autre, qui ne serait plus que «littérature », «jeux de mots ». N'est-ce pas, finalement, le même vieux débat entre une écriture explorant le monde, et une écriture s'arrêtant aux mots ? Le renouvellement de la littérature de voyage ne serait-elle, en fait, que la réaffirmation d'une écriture moins éprise de littérature, et davantage de voyage ?

Cependant, certains écrivains contemporains, comme Olivier Rolin, refusent une opposition tranchée entre littérature de voyage et littérature tout court. 
Il n'y a pas plus de littérature de voyage, me semble-t-il, qu'il n'y a d'agences de littérature. Et puis, il ne me plait pas que ce retour au vert paradis des émerveillements enfantins ait été prôné, en France, pour tenter de prendre une très explicite revanche sur le supposé «terrorisme» d'une tradition qui prétendait ne pas laisser cette bonne vieille chose romanesque à l'écart de la philosophie, des sciences humaines, de la pensée critique. (ROLIN, $1997: 9$ )

Pour Olivier Rolin, il n’y a pas opposition entre littérature et voyage. Il n’y a que littérature. Il souligne même, dans la suite de son texte, toutes les similitudes qu'il est possible de relever entre le voyage et le travail de l'écrivain.

Parmi ces dernières, il en est une qui, plus particulièrement, touche à l'écriture. Le voyage met en contact avec des choses nouvelles. Cela était vrai au XVIe siècle quand, débarquant au Brésil, Jean de Léry se trouvait confronté à une faune, une flore, une humanité encore inconnues. Mais, contrairement à ce que peut affirmer Lévi-Strauss, ça l'est toujours au XXe siècle : en effet, pour Olivier Rolin, voyager, c'est encore « se trouver sans cesse confronté à des situations, des mœurs, des choses nouvelles » (ROLIN, 1997 : 15). Or, cet confrontation avec le nouveau met le voyageur au défi de nommer ce qui n'a pas de nom, de trouver le mot juste pour évoquer des situations qui, auparavant, n’ont jamais été évoquée dans sa langue. Olivier Rolin va même plus loin : le voyageur ne saurait voir tant qu'il n'a pas trouver le mot juste pour décrire ce qu'il voit.

On ne voit vraiment une chose que lorsqu'on a trouvé les mots pour la dire. Ce sont les mots, les mots difficiles, qu'il faut aller chercher très loin, parfois, dans les profondeurs de la langue, qui suscitent et durcissent l'image d'une chose, comme le cuivre martelé, repoussé, fixe une figure. L'œil écrit, ou plutôt : la plume voit. Je ne vois, littéralement, le ciel que lorsque j'ai trouvé, pour le décrire, autre chose que : il est bleu, ou gris. Homère nous fait voir la mère bachique, sanglante, sombre, nauséeuse, la mer vivier de monstres, reflétant des soleils bas etc., lorsqu'il la dit oinops, couleur de vin. (ROLIN, $1997: 15$ )

Le voyageur, pour voir, doit partir en quête du mot juste, chercher dans les lointains de sa langue ce qui lui permettra de rendre compte des lointains géographiques. Sans le mot, il ne saurait voir la chose. Sans la «plume », le voyageur n'est qu'un « œil » aveugle. Voir, c'est écrire. Le voyage est une incitation à l'écriture. Or, l'usage commun de certains mots ne saurait montrer une réalité nouvelle. «Pour voir ce qu'on n'a pas vu, fixer l'image de choses, de mœurs, de situations nouvelles, il faut inventer, sinon des mots nouveaux [...], au moins un nouvel usage des mots, un nouvel arrangement des mots et des phrases » (ROLIN, 1997 : 15). Mais alors, par cette recherche du mot exact, de l'adjectif le plus juste, d'un assemblage 
nouveau des mots, le voyageur opère sur le langage le même travail que le poète. Quand il lui faut, comme Homère, trouver une épithète aussi inattendue que «vineuse » pour décrire la mer sombre ou, comme Francis Ponge (autre exemple que donne Olivier Rolin), aussi surprenant que «sacripant » pour décrire le rose du Sahel (PONGE, 1967 : 21), l'explorateur de l'espace devient explorateur de la langue, le voyageur se fait poète. C'est d'ailleurs, quoiqu'elle en dise, un travail de ce genre sur la langue qu'opère Ella Maillart quand elle cherche les mots qui lui permettraient de donner une idée juste du bleu turquoise, ce bleu qu'elle finit «appeler "bleu d'Asie centrale" en souvenir d'un lac glaciaire des Tian Shan qui déployait cette radieuse saturation bleu ciel à reflets verts » (p. 132).

Aussi, la « langue extrêmement précise » dont parle Nicolas Bouvier à propos d'Ella Maillart n'est-elle pas si loin qu'on pourrait le penser, au premier abord, de la langue du poète. Peut-être, d'ailleurs, n'est-elle jamais aussi poétique que lorsqu'elle se fait plus « précise ». Par des voies indirectes, et selon des exigences propres au voyage, même sans en avoir l'intention ni la «prétention », le voyageur fidèle à ses exigences de précisions finit tout de même par faire œuvre littéraire. Ainsi, bien que la Lettre de Caminha ne soit pas écrite dans un but littéraire, et que son auteur, comme nous l'avons vu, se dénie toute qualité d'écrivain, son texte, comme le rappelle Leyla Perrone-Moisés, «é um texto de autor, assinado por um talentoso escrivão de bordo, cujas qualidades têm sido justamente reconhecidas como as de um verdadeiro escritor »(PERRONE-MOISÉS, 1992 : 89). Aussi, sans que son récit ne perde sa valeur de témoignage, Caminha a malgré tout réussi à faire de la littérature et, de talentueux « escrivão », à se hisser au niveau d'« escritor ».

Pourquoi alors les auteurs de récits de voyage refusent-ils d'être pris pour des écrivains ? C'est, on l'a vu, pour qu'on ne doute pas de leur sincérité : qu'on ne doute pas que ce qu'ils disent est bien réel. Or, les études linguistiques ont montré que le langage ne peut pas représenter le réel. Cependant, comme le souligne Roland Barthes, « de ce qu'il n’y a point de parallélisme entre le réel et le langage, les hommes ne prennent pas leur parti, et c'est ce refus, peut-être aussi vieux que le langage lui-même, qui produit, dans un affairement incessant, la littérature » (BARTHES, 1978 : 22). Aussi la question du réalisme en littérature n'est-elle plus, comme le note Philippe Hamon, «comment la littérature copie-t-elle la réalité ?, [...] question devenue sans intérêt », mais «comment la littérature nous fait-elle croire qu'elle copie la réalité ?»(Collectif, 1982: 130) : autrement dit, par quels moyens les écrivains parviennent-ils à convaincre qu'il leur est, malgré les limites propres aux langage, possible de représenter le réel ? Et l'on pourrait imaginer avec Barthes d'écrire une histoire de 
la littérature à partir de ces moyens mis en œuvre, « des expédients verbaux, souvent très fous, dont les hommes ont usé pour réduire, apprivoiser, nier, ou au contraire assumer ce qui est toujours un délire, à savoir l'inadéquation fondamentale du langage et du réel » (BARTHES, $1970: 22)$.

Or, la littérature de voyage se trouve, plus que toute autre, confrontée à cette «inadéquation fondamentale », avec d'ailleurs cette difficulté supplémentaire qu'il lui faut rendre compte d'une réalité étrangère à laquelle les mots dont elle dispose ne sont pas forcément adaptés. C'est peut-être en cela que la littérature de voyage, bien que longtemps en marge de l'institution littéraire, se trouve pourtant au cœur de la littérature : à la fois, l'étrangeté de ce qu'il voit pousse le voyageur à rendre compte du réel par le langage ; mais c'est alors qu'il se trouve confronté à l'impossibilité propre au langage de rendre compte du réel. Comme tout écrivain, ayant « le réel pour objet de désir », force lui est de constater que c'est là «le désir de l'impossible » (BARTHES, 1970:23) - sans pour autant qu'il ne se résigne au silence.

Aussi, l'absence de prétention littéraire n'est-elle qu'une posture: le moyen, l' «expédient verbal», par lequel les auteurs de récits de voyage ont cherché, au long des siècles, à convaincre leur lecteur qu'il leur était possible de représenter le réel par l'écriture. Mais il ne faudrait pas confondre ce qui, d'une part, est une pétition de principe - qui relève de la captatio benevolentia - avec ce qui se produit réellement dans un récit de voyage, dont les auteurs, du moins les plus talentueux d'entre eux, n'ont sans doute jamais rien fait d'autre que de la littérature.

À la fin du XXe siècle, le retour de la littérature de voyage ne se fait donc pas forcément contre la littérature. Au contraire, il se doit peut-être - grâce aux recherches de la linguistique - à une prise de conscience du caractère hautement littéraire de l'écriture viatique qui relève, comme le suggère Olivier Rolin, depuis toujours, de la littérature tout court. La littérature de voyage, après avoir rejeté toute prétention littéraire a peut-être bien fini par se fondre complètement dans la littérature qui n'est pas «un corps ou une suite d'œuvres, ni même un secteur de commerce ou d'enseignement, mais le graphe complexe des traces d'une pratique : la pratique d'écrire »(BARTHES, 1978 : 16). 


\section{ANNEXES}

Annexe 1a : Les itinéraires d'Ella Maillart, 1930-1940.

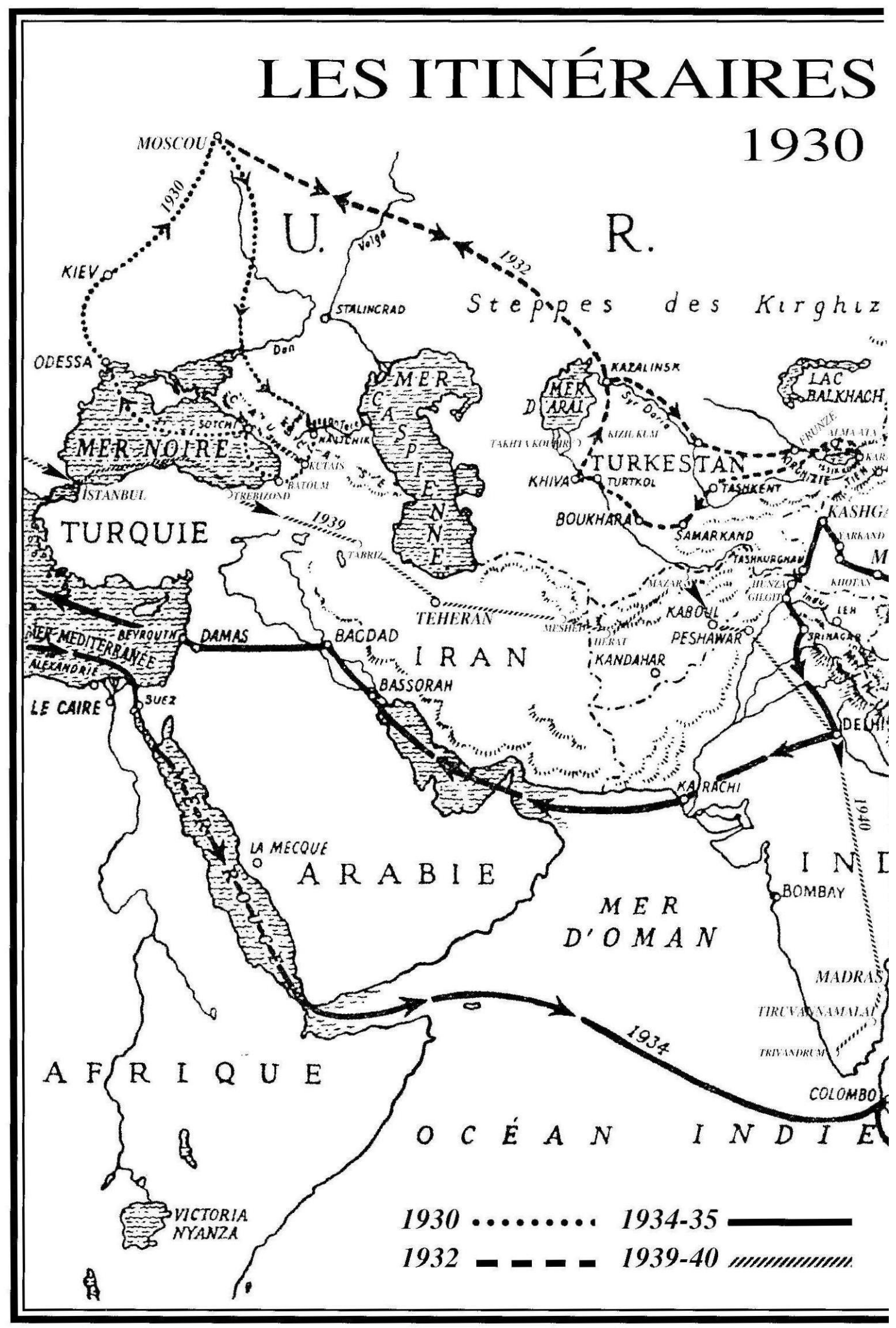




\section{D'ELLA MAILLART \\ 1940}

S., 5, S. 7 $\mathrm{A}$ MONOOLILERCA

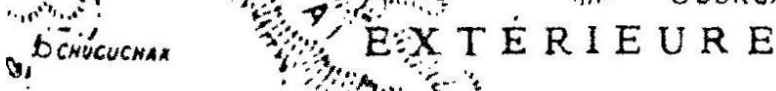
8 son

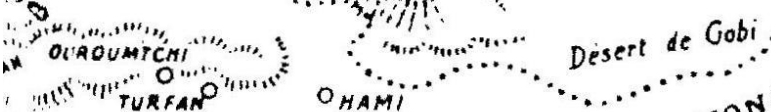

INKIANG

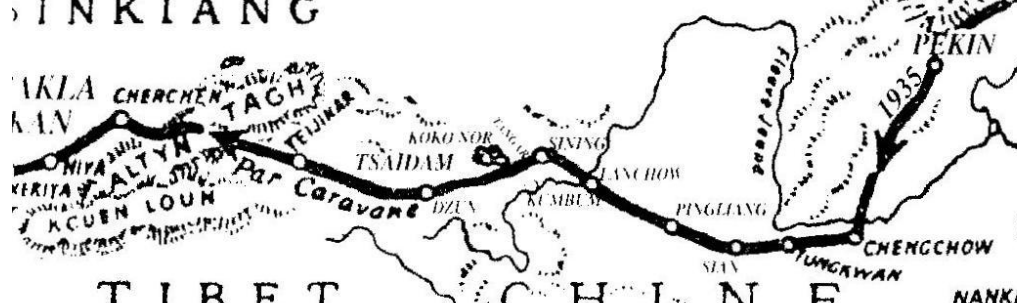

T I B E T C $\mathrm{H} I \mathrm{~N} \mathrm{E}$

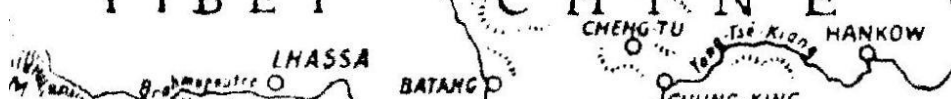

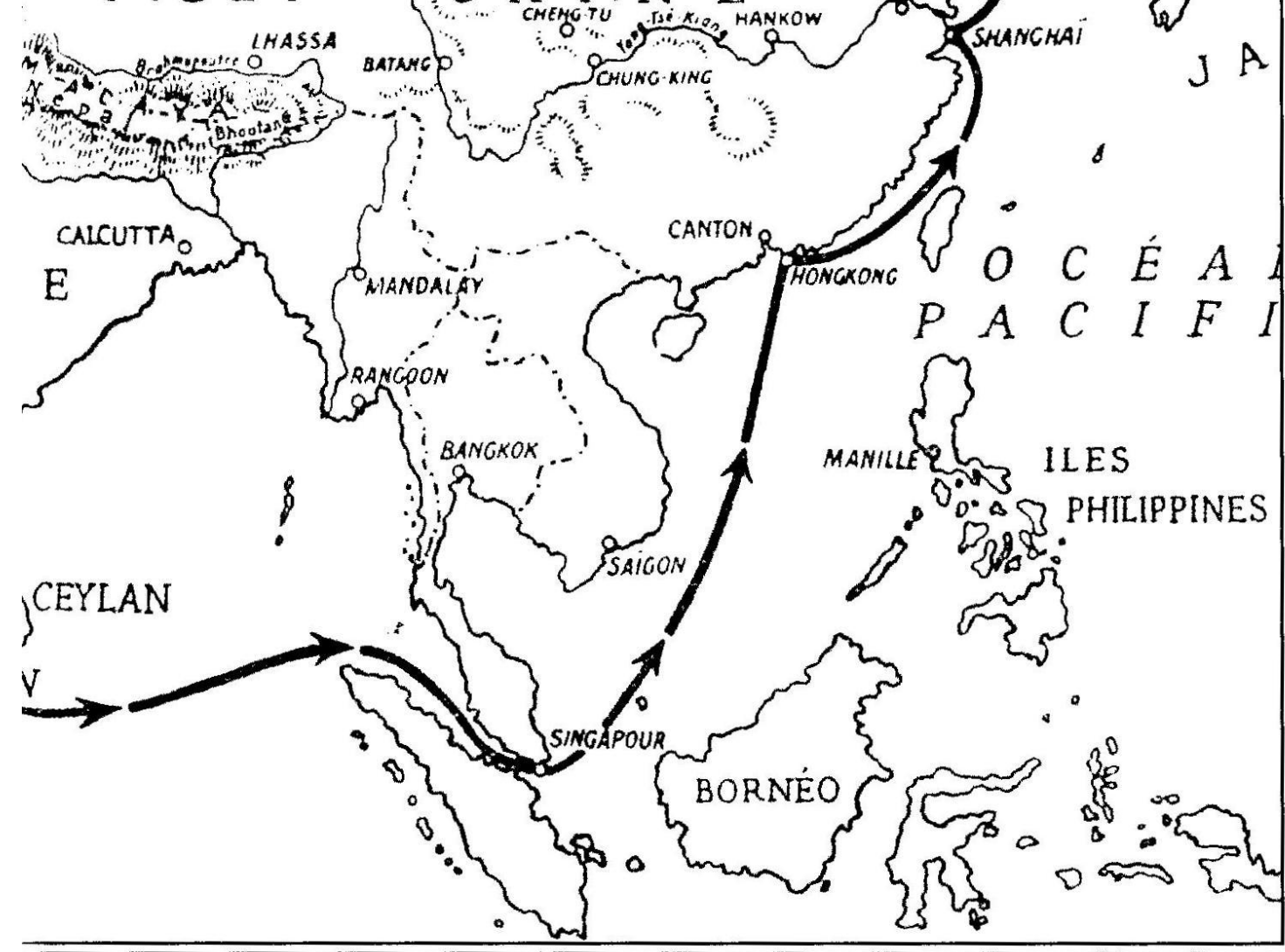


Annexe 2:

Annemarie Schwarzenbach/Christina (à gauche) et Ella Maillart/Kini (à droite)

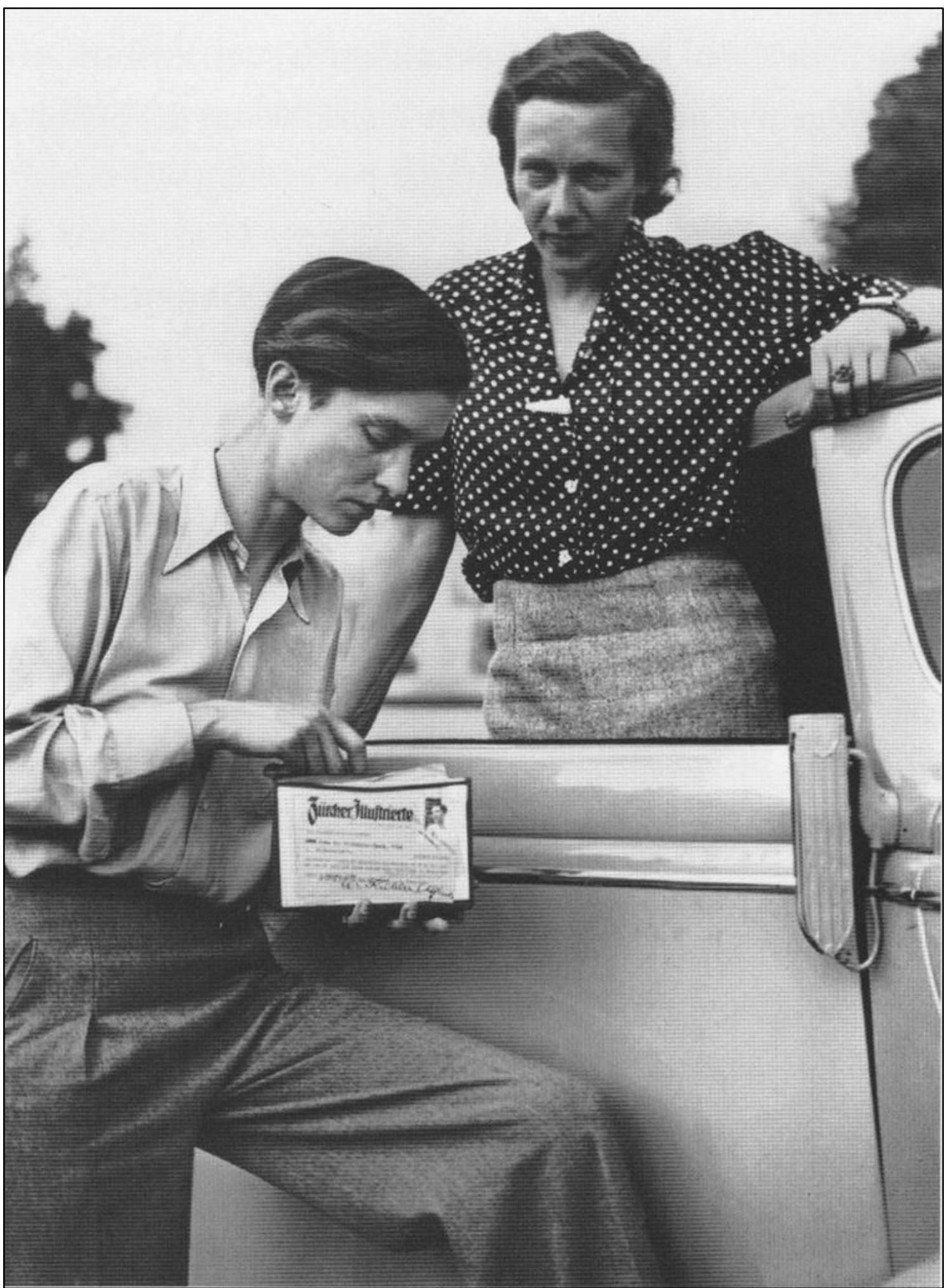

Gilbert Meylan: Annemarie Schwarzenbach - tenant à la main sa carte de presse de la Zürcher Illustrierte - et Ella Maillart, avant de partir pour 1'Afghanistan, Genève, 6 juin 1939, in Bleu immortel, p. 85. 
Annexe 3 :

Le tchador

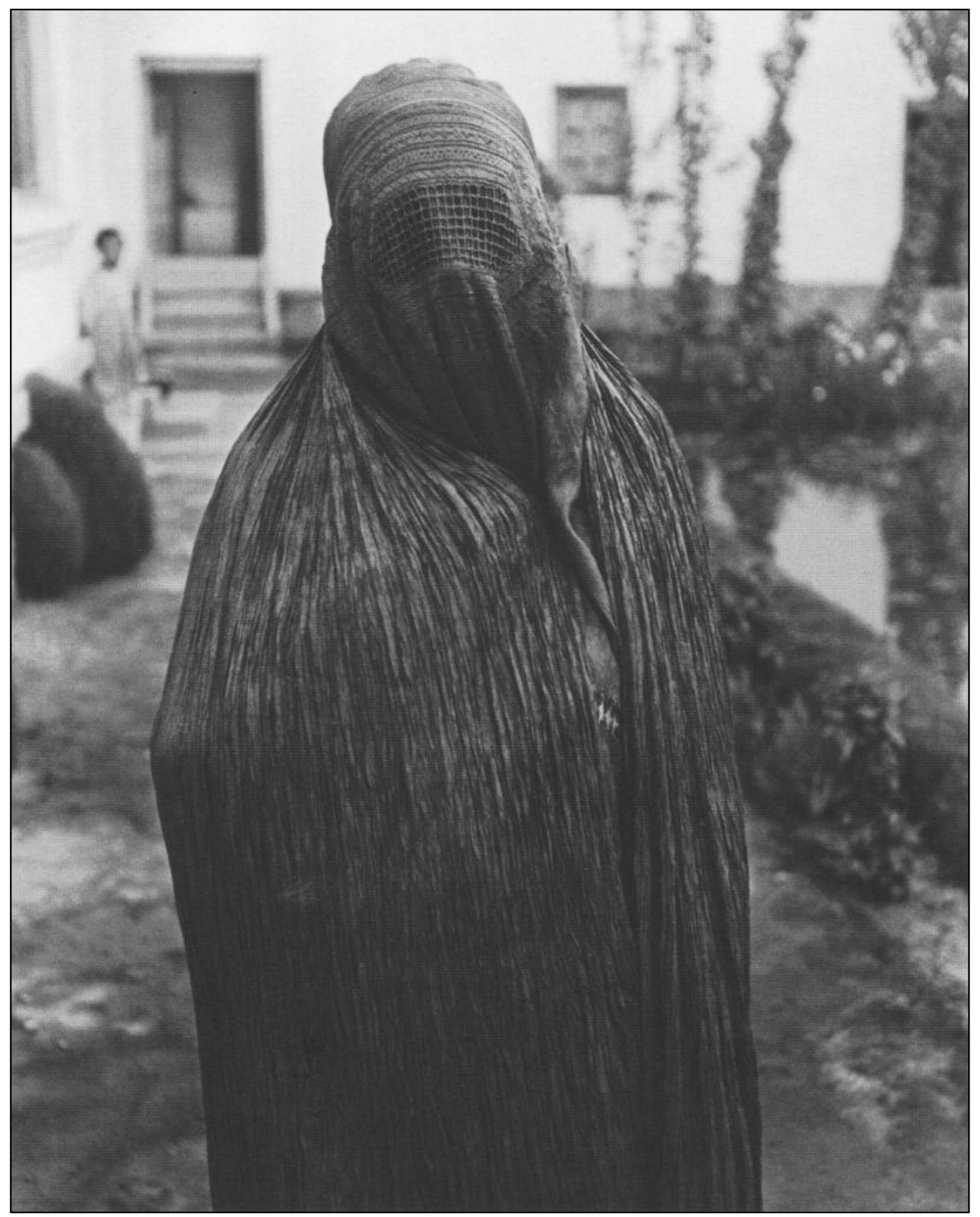

Ella Maillart : Kaboul/Afghanistan, septembre/octobre 1939, in Bleu immortel, p. 157. 
Annexe 4 :

Ouvrier afghan à Poul-i-Khumri.

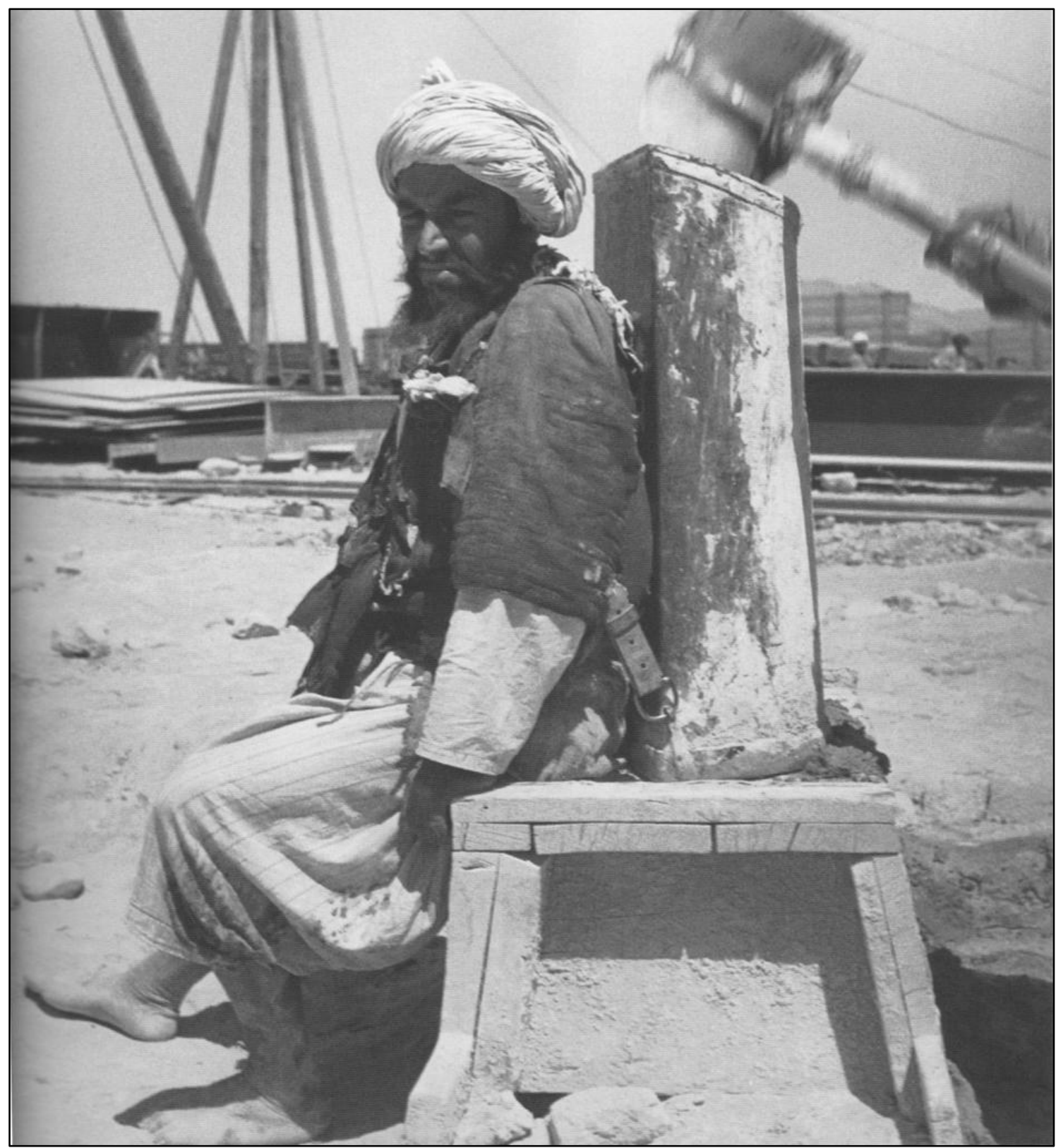

Annemarie Schwarzenbach : Centrale électrique à Poul-i-Khumri/Afghanistan, août 1939, in Bleu immortel, p. 145. 


\section{BIBLIOGRAPHIE}

\section{Euvres d'Ella Maillart}

MAILlART, Ella. Parmi la jeunesse russe. De Moscou au Caucase (1932), Paris : Payot \& Rivages, 2003a. 216 p. (PBP/Voyageurs).

. Des Monts Célestes aux sables Rouges (1934), Paris : Payot \& Rivages, 2001a. 357 p. (PBP/Voyageurs).

- Oasis interdites (1937), Paris: Payot \& Rivages, 2002a. 333 p. (PBP/Voyageurs).

. Gypsy Afloat (1942), La Vagabonde des mers (1991), Paris : Payot \& Rivages, 2002b. 365 p. (PBP/Voyageurs).

. Cruises and Caravans (1942), Croisières et caravanes (1951), Paris :

Payot \& Rivages, 2001b. 234 p. (PBP/Voyageurs).

. The Cruel Way (1947), La Voie cruelle (1952), Paris : Payot \& Rivages, 2001c. 315 p. (PBP/Voyageurs).

. Ti-Puss (1951), Ti-Puss ou l'Inde avec ma chatte (1979), Paris : Payot \& Rivages, 2002c. 237 p. (PBP/Voyageurs).

- La Vie immédiate. Photographies, textes de Nicolas Bouvier, Paris :

Payot \& Rivages, 1991. 197 p.

. Sur les routes de l'Orient. Photographies, textes de Daniel Girardin, Arles : Actes Sud, 2003b. 159 p.

. Cette réalité que j'ai pourchassée, Correspondance, Genève : Zoé, 2006, $168 \mathrm{p}$. Envoyée spéciale en Mandchourie, Genève : Zoé, 2009. 144 p.

\section{Autres récits de voyages}

BARTHES, Roland. L'Empire des signes, Paris : Le Seuil, 1970. 157 p. (Points Essais).

BOUGAINVILLE, Louis-Antoine (de). Voyage autour du monde sur la frégate la Boudeuse et la Flûte l'Étoile (1766), Paris : La Découverte, 1997. 292 p. (La Découverte/Poche). BOUVIER, Nicolas. Euvres, Paris : Gallimard, 2004. 1418 p. (Quarto). 
MAILLART, Ella, SCHWARZENBACH, Annemarie. Bleu immortel. Voyages en Afghanistan, Genève : Zoé, 2003. 269 p.

CASTRO, Silvio. A Carta de Pero Vaz de Caminha, o descobrimento do Brasil, Porto Alegre: L\&PM, 1985. 156 p. (Pocket).

CLAUDEL, Paul. Connaissance de l'Est, Paris : Gallimard, 1900. 336 p. (Poésie/Gallimard). FLAUBERT, Gustave. Voyage en Orient, Paris : Gallimard, 2006. 738 p. (Folio Classique).

FLEMING, Peter. Un aventurier au Brésil, Au fond du Mato Grosso sur les traces du Colonel Fawcett (1933), Paris : Phébus, 1990. 376 p.

Courrier de Tartarie (1936), Paris : Phébus, 1989. 423 p. (Libretto).

GIDE, André. Voyage au Congo, Paris : Gallimard, 1928. 554 p. (Folio).

LÉRY, Jean (de). Histoire d'un Voyage faict en la terre du Brésil (1580), précédée d'un entretien avec Claude Lévi-Strauss «Sur Jean de Léry », édition de Frank Lestringant, Paris : Le Livre de Poche, 1994. 670 p. (Bibliothèque classique).

LÉVI-STRAUSS, Claude. Tristes Tropiques, Paris : Plon, 1955. 504 p. (Terre Humaine / Poche).

MICHAUX, Henri. Ecuador (1929) in Euvres complètes, vol. 1, Paris : Gallimard, 1998. p. 137-243 (La Pléiade).

- Un Barbare en Asie (1933) in Euvres complètes, vol. 1, Paris : Gallimard, 1998. p. 274-409 (La Pléiade).

ROLIN, Olivier. Mon galurin gris: Petites géographies, Paris : Le Seuil, 1997. 266 p. (Fiction \& Cie).

SEGALEN, Victor. Équipée (1929), Paris : Gallimard, 1983. 147 p. (L'Imaginaire).

STADEN, Hans. Nus, féroces et anthropophages (1557), Paris: Métailié, 1979. 259 p. (Suites).

SCHWARZENBACH, Annemarie. Où est la terre des promesses? Avec Ella Maillart en Afghanistan (1939-1940), Paris : Payot \& Rivages, 2002. 203 p. (PBP/Voyageurs).

THEVET, André. Les Singularités de la France Antarctique (1557), édition de Frank Lestringant, Paris : Chandeigne, 1997. 445 p. (Magellane).

3. Sur la littérature de voyage

BOUVIER, Nicolas. L'Echappée belle. Eloge de quelques pérégrins, 5 éd., Genève : Métropolis, 2000. 162 p.

BUARQUE DE HOLANDA, Sérgio. Visão do Paraíso (1959), 6 éd., São Paulo : Brasiliense, 1996. $365 \mathrm{p}$. 
COGEZ, Gérard. Les écrivains voyageurs au XXe siècle, Paris : Seuil, 2004. 229 p. (Points Essais).

COLLECTIF. Pour une littérature voyageuse, Paris : Complexe, 1992. 214 p. (Le regard littéraire).

CRISTOVÃO, Fernando. "Teoria da Literatura de viagem" in Condicionantes culturais da literatura de viagens: Estudos e bibliografias, Lisbonne: Cosmos, 1999, p. 15-52.

DOIRON, Normand. L'art de voyager, Le déplacement à l'époque classique, Paris : Klincksieck, 1995. 258 p.

FORSDICK, Charles. Oasis interdites, Ella Maillart, Carouge-Genève : Zoé, 2008. 113 p. (Etudes Littéraires).

GOMEZ-GÉRAUD Marie-Christine, ANTOINE, Philippe (org.). Roman et récit de voyage, Paris : PUPS, 2001. 252 p. (Imago Mundi).

HAMBURSIN, Olivier (org.). Récits du dernier siècle des voyages, De Victor Segalen à Nicolas Bouvier, Paris : PUPS, 2005. 262 p. (Imago Mundi).

HARTOG, François. Le Miroir d'Hérodote, éd. revue et augmentée, Paris : Gallimard, 2001. 581 p. (Folio Histoire).

LAPOUGE, Gilles. L'encre du voyageur, Paris : Albin Michel, 2007. 259 p.

LESTRINGANT, Frank. Jean de Léry ou L'invention du sauvage, Essai sur l'Histoire d'un Voyage faict en la terre du Brésil, Paris: Honoré Champion, 1999. 221 p. (Unichamp).

MICHAUX, Henri. «Les poètes voyagent » in Passages (1950), Euvres complètes, vol. 2, Paris : Gallimard, 2001. p. 307-309 (La Pléiade).

MOUREAU, François, org. Le Second voyage ou le déjà-vu, Paris : Klincksiek, 1996. 142 p.

RIDON, Jean-Xavier. Le voyage en son miroir, Essai sur quelques tentatives de réinvention du voyage au $20^{e}$ siècle, Paris : Kimé, 2002. 172 p.

PERRONE-MOISÉS, Leyla. Vinte Luas: Viagem de Paulmier de Gonneville ao Brasil: 15031505, São Paulo : Companhia das Letras, 1992. 186 p.

PERRONE-MOISÉS, Leyla. «Vérité et fiction dans les premières descriptions du Brésil » in SEIXO, Maria Alzira (org.). A Viagem na literatura, Mem Martins, 1997, p. 83-102.

SEIXO, Maria Alzira et ABREU, Graça (org.), Les récits de voyages : typologie, historicité, Lisbonne : Cosmos, 1998. 304 p. (Viagem).

TODOROV, Tzvetan. La Conquête de l'Amérique : La question de l'autre, Paris : Le Seuil, 1982. 329 p. (Points Essais). 
TODOROV, Tzvetan. Nous et les autres, La Réflexion française sur la diversité humaine, Paris : Le Seuil, 1989. 538 p. (Points Essais).

WOLFZETTEL, Friedrich. Le discours du voyageur, Paris : PUF, 1996. 334 p. (Perspectives littéraires).

\section{Autres textes}

AUERBACH, Erich. Mimésis, la représentation de la réalité dans la littérature occidentale (1946), Paris : Gallimard, 1968. 559 p. (Tel).

BARTHES, Roland. «Écrivains et écrivants » in Essais critiques, Paris : Le Seuil, 1964. p. 152-159 (Points Essais).

BARTHES, Roland. Leçon, Paris : Le Seuil, 1978. 45 p. (Points Essais).

BENVENISTE, Émile. «Nature du signe linguistique » in Problèmes de linguistique générale, vol. 1, Paris : Gallimard, 1966, p. 49-55. (Tel).

BIBLE. Français. La Bible. Trad. Lemaître de Sacy, éd. de Philippe Sellier. Paris : Robert Laffont, 1990. (Bouquins).

FOUCAULT, Michel. Les Mots et les choses, Paris : Gallimard, 1966. 400 p. (Tel).

LEJEUNE, Patrick. Le Pacte autobiographique, éd. augmentée, Paris : Seuil, 1996. 381 p. (Points Essais).

MONTAIGNE, Michel (de). Les Essais (1595), éd. de Jean Balsamo, Michel Magnien et Catherine Magnien-Simonin, Paris : Gallimard, 2007. 1975 p. (La Pléiade).

PESSOA, Fernando. Livro do desassossego, éd. de Richard Zenith. São Paulo : Companhia das Letras, 1999. $536 \mathrm{p}$.

PONGE, Francis. Méthodes, Paris : Gallimard, 1961. 248 p. (Folio Essais).

ROUSSEAU, Jean-Jacques. Discours sur l'origine et les fondements de l'inégalité parmi les hommes (1754), éd. de Jean Starobinski, Paris : Gallimard, 1969. 284 p. (Folio Essais).

ROUSSET, Jean. Leurs yeux se rencontrèrent. La scène de première vue dans le roman, Paris : Corti, 1984. 216 p.

TODOROV, Tzvetan (org.). Littérature et réalité, Paris : Le Seuil, 1982. 181 p. (Points Essais). 